\title{
Nonlocal magnetization dynamics in ferromagnetic heterostructures
}

\author{
Yaroslav Tserkovnyak \\ Lyman Laboratory of Physics, Harvard University, Cambridge, Massachusetts 02138, USA \\ Arne Brataas \\ Department of Physics, Norwegian University of Science and Technology, \\ N-7491 Trondheim, Norway
}

Gerrit E. W. Bauer

Kavli Institute of NanoScience, Delft University of Technology, 2628 CJ Delft, The Netherlands

Bertrand I. Halperin

Lyman Laboratory of Physics, Harvard University, Cambridge, Massachusetts 02138, USA

(Published 1 December 2005)

\begin{abstract}
Two complementary effects modify the GHz magnetization dynamics of nanoscale heterostructures of ferromagnetic and normal materials relative to those of the isolated magnetic constituents. On the one hand, a time-dependent ferromagnetic magnetization pumps a spin angular-momentum flow into adjacent materials and, on the other hand, spin angular momentum is transferred between ferromagnets by an applied bias, causing mutual torques on the magnetizations. These phenomena are manifestly nonlocal: they are governed by the entire spin-coherent region that is limited in size by spin-flip relaxation processes. This review presents recent progress in understanding the magnetization dynamics in ferromagnetic heterostructures from first principles, focusing on the role of spin pumping in layered structures. The main body of the theory is semiclassical and based on a mean-field Stoner or spin-density-functional picture, but quantum-size effects and the role of electron-electron correlations are also discussed. A growing number of experiments support the theoretical predictions. The formalism should be useful for understanding the physics and for engineering the characteristics of small devices such as magnetic random-access memory elements.
\end{abstract}

\section{CONTENTS}

I. Introduction
A. Preliminaries
B. Nonlocal exchange coupling and giant magnetoresistance
C. Landau-Lifshitz-Gilbert phenomenology
D. Current-induced magnetization dynamics
E. Spin emission by excited ferromagnets

II. Scattering-Theory Approach to Magnetoelectronics
A. Magnetoelectronic dc circuit theory
B. Interfacial and thin-film conductance matrices
C. Time-dependent theory

III. Spin Emission by Coherently Precessing Ferromagnets
A. Parametric spin pumping
B. Rotating-frame analysis
C. FMR-operated spin battery

IV. Gilbert-Damping Enhancement
A. Ideal spin sinks
B. Diffuse systems
C. Enhanced Gilbert damping in spin valves: First-principles calculations versus experiment

V. Dynamic Exchange Interaction
A. Magnetic bilayers
B. Magnetic superlattices
C. Large-angle motion in biased spin valves

VI. Linear-Response Approach
A. Heterostructures

B. Bulk damping 1410

VII. Miscellaneous

1412

$\begin{array}{ll}\text { A. Quantum-size effects } & 1412 \\ \text { 1. Ultrathin magnetic layer } & 1412\end{array}$

2. Ultrathin normal spacer 1414

B. Spin-orbit coupling 1415

C. Inhomogeneous magnetization dynamics 1415

D. Electron-electron interactions 1416

VIII. Summary and Outlook 1417

Acknowledgments 1418

References 1418

\section{INTRODUCTION}

\section{A. Preliminaries}

A ferromagnet is a symmetry-broken state in which a majority of electrons point their spin into a certain common direction below critical temperatures as high as $1000 \mathrm{~K}$. The robustness of the magnetic order and the permanence of a given magnetization direction against elevated temperatures and external perturbations have been employed in applications as diverse as compass needles, refrigerator-door stickers, and memory devices.

In spite of its stability, ferromagnetism is neither rigid nor static. Depending on sample size and anisotropies due to crystal field and sample shape, a single-domain 
ferromagnet is often unstable with respect to a domain structure that lowers the macroscopic magnetic energy. Thermal fluctuations reduce the macroscopic moment until it completely vanishes at the critical temperature $T_{c}$. At temperatures sufficiently below $T_{c}$, the internal dynamics of the ferromagnet are dominated by lowenergy transverse fluctuations of the magnetization, socalled spin waves or magnons, that are the magnetic equivalence of phonons in a lattice. Classical coarsegrain computer simulations of the detailed position- and time-dependent magnetization ("micromagnetism") describe these phenomena well (Brown, 1963; Miltat et al., 2002).

When magnetic grains become sufficiently small, the exchange stiffness renders domain structures that are not energetically favorable and a single-domain picture is adequate. When the ferromagnet is exposed to a uniform driving field, the macroscopic magnetization dynamics may then be dominated by a collective precession of the entire ferromagnetic order parameter. Restricting the ferromagnetic degrees of freedom to this mode is often referred to as the macrospin model. In infinite ferromagnetic media, low-energy spin waves resemble symmetry-restoring Goldstone modes, but in real life, the spin-rotational symmetry is broken by magnetic anisotropies caused by magnetic dipolar fields or by crystal-field spin-orbit interactions. In thermodynamic equilibrium, the macrospin then points in a certain fixed direction with small thermal fluctuations around it. The ferromagnet can still be coerced into motion by applying external magnetic fields at a finite angle to the magnetization direction. The system then moves in response, trying to minimize its Zeeman energy. The compass needle, a freely suspended single-domain ferromagnet with a sufficiently high anisotropy (coercivity), does this by alignment of its lattice. In this review, we are interested in mechanically fixed magnets whose magnetic moments move in the presence of external and anisotropy effective magnetic fields, as well as applied electric currents. Viscous damping processes are required to achieve a reorientation (switching) of the magnetization, if, for example, a magnetic-field direction is suddenly changed. Minimization of this finite switching time by engineering magnetic anisotropies and magnetization-damping rates is an important goal in the design of fast magnetic memories. When the applied magnetic fields are large enough to surmount the anisotropies, the magnetization can be reversed, often by large amplitude and complex trajectories, even in the simple macrospin model. At finite temperatures, the magnetization reorientation becomes probabilistic and is described by a Fokker-Planck equation on the unit sphere (Brown, 1963).

In the last two decades, a new subdiscipline in the field of magnetism has risen that is devoted to the studies of heterostructures of ferromagnets $(F)$ with normal metals $(N)$ and, to a lesser extent, semiconductors and superconductors. Especially magnetoelectronics, the science and technology directed at understanding and utilizing the transport properties of layered structures of ferromagnetic and normal metals, has grown into a mainstream topic of condensed-matter physics. Its attraction derives from large effects at room temperature that can be understood easily in terms of transparent physics and that have already found numerous applications. Two crucial discoveries in magnetic multilayers still reverberate in recent research, viz., the nonlocal oscillatory exchange coupling by Grünberg et al. (1986) and the giant magnetoresistance (GMR) by Baibich et al. (1988) and Binasch et al. (1989). Exchange coupling through a metallic spacer favors an antiparallel coupling between ferromagnetic layers for certain spacer thicknesses, depending on the occupation of spin-polarized quantum-well states. It is therefore a quantuminterference effect sensitive to defect scattering, which vanishes exponentially with increasing spacer-layer thickness. GMR is a phenomenon in which the resistance of multilayers is reduced when the magnetic configuration is forced from antiparallel to parallel by an applied magnetic field. In disordered multilayers, it is a semiclassical transport effect that can be understood in terms of a diffusion equation (Camley and Barnas, 1989; Valet and Fert, 1993). In a configuration in which the currents are oriented perpendicular to the interface planes (CPP) (Pratt et al., 1991; Gijs et al., 1993; Gijs and Bauer, 1997), electrical transport can be mapped onto a two-channel (spin-up and spin-down) resistor model in which interface and bulk resistances for a fixed spin are simply connected in series. The spin-relaxation processes are usually modeled by a local finite-resistance link connecting the spin-up and spin-down circuits.

Initially, the community focused its attention on stationary magnetic states, like those responsible for the magnetoresistance in metallic and tunneling structures with applied dc bias. This has changed drastically in recent years. The main catalyst was the experimental verifications of an earlier prediction by Berger (1996) and Slonczewski (1996) that electric currents can cause a reorientation of the ferromagnetic order in multilayer structures. Tsoi et al. (1998) experimentally demonstrated magnetization precession in $(\mathrm{Co} \mid \mathrm{Cu})_{N}$ multilayers by currents injected by a point contact, whereas Myers et al. (1999) observed switching in the orientation of magnetic moments in $\mathrm{Co}|\mathrm{Cu}| \mathrm{Co}$ sandwich structures by perpendicular electric currents ("CPP spin valves"). Much earlier, a coupling between a dynamic ferromagnetic magnetization and spin accumulation in adjacent normal metals had been postulated by Janossy and Monod (1976) and Silsbee et al. (1979). These authors demonstrated that microwave transmission through normal-metal foils facilitated by conduction-electron spin transfer is significantly enhanced by ferromagneticlayer coating.

This review covers the developments in the understanding of the magnetization dynamics in heterostructures of ferromagnets and normal conductors in the last five years or so. We believe that the time is ripe, since from a microscopic point of view, much of the basic physics is well understood. A consistent and coherent picture has evolved that is based on the diffusion equa- 
tion for the bulk transport in metallic ferromagnets and normal conductors with quantum-mechanical boundary conditions at possibly sharp interfaces between them. Noncollinearity of the magnetization directions in structures with more than one magnet is an essential ingredient in order to understand the physics. We focus here on explicitly dynamic effects, referring to a separate article (Brataas, Bauer, and Kelly, 2005) for the static transport properties of magnetoelectronic circuits and devices. The present review follows a self-consistent approach extending the static magnetoelectronic circuit theory to time-dependent phenomena. It provides a framework for including on an equal footing two physical effects that are two sides of one coin, viz., the spin-transfer torque induced by applied currents (Slonczewski, 1996) and the spin pumping by moving ferromagnets into adjacent conductors (Tserkovnyak et al., 2002a). The theory is derived from microscopic principles and the material-dependent input parameters are thus accessible to ab initio calculations. We concentrate on quasi-onedimensional models corresponding to, e.g., layered pillar structures. With few exceptions, we do not attempt accurate modeling of concrete device structures and deviations of the magnetization dynamics from the macrospin model, although the theory discussed in this review can be readily generalized to treat such situations. We focus on adiabatic effects to lowest order in the characteristic Larmor frequency. In spite of these limitations, the agreement with various experiments is found to be gratifying.

Effects beyond such a model certainly may cause observable phenomena. For example, the quantum interference that leads to inversion of the magnetoresistance in high-quality tunnel junctions cannot be treated by the diffusion equation (Yuasa et al., 2002). Nonlinearities require numerical simulations or a stability analysis based on the theory of dynamic systems that are outside our scope (Valet, 2004). High temperatures and currents can best be treated by stochastic methods beyond the present review (Apalkov and Visscher, 2004; Li and Zhang, 2004b) but the input parameters of such approaches are provided here. The current-induced dynamics of domain walls ( $\mathrm{Li}$ and Zhang, 2004a; Tatara and Kohno, 2004; Barnes and Maekawa, 2005, and references therein) are also beyond the macrospin considerations central to this review. Whereas the spintransfer-torque-induced dynamics are a crucial ingredient, space constraints force us to abandon complete coverage of the numerous experiments published recently.

Throughout the review, we focus on self-consistent effects arising from the time-dependent ferromagnetic exchange field felt by itinerant carriers in the mean-field picture. We take the spin-orbit interaction into account only in terms of a phenomenological spin-flip relaxation time, Secs. II-V, but consider it more seriously in Secs. VI.B and VII.B. Most results are not material specific, but unless specified otherwise we have heterostructures of transition-metal ferromagnets (and its alloys) with noble or other simple normal metals in mind.
The main body of this review is organized as follows. Section I introduces several basic concepts that we rely on in the remainder of the article, which is primarily aimed at nonspecialists. Section II is a brief but in-depth discussion of the magnetoelectronic circuit theory (see also Brataas, Bauer, and Kelly, 2005), which is then generalized in Sec. III to dynamic problems by means of the spin-pumping concept. Sections IV and V, respectively, discuss Gilbert damping and dynamic ferromagnetic exchange in heterostructures, which are mediated by spin pumping and spin-transfer torques. Section VI is devoted to an alternative linear-response view of the nonlocal magnetization dynamics, and Sec. VII treats several special topics before we conclude the article with a summary and outlook in Sec. VIII.

\section{B. Nonlocal exchange coupling and giant magnetoresistance}

The discovery that the energy of magnetic multilayers made from alternating ferromagnetic and normal-metal films depends on the relative direction of the individual magnetizations (Grünberg et al., 1986) is perhaps the most important in magnetoelectronics. The existence of the antiparallel ground-state configuration at certain spacer-layer thicknesses was essential for the subsequent discovery of the giant magnetoresistance (Baibich et al., 1988; Binasch et al., 1989). Adjacent ferromagnetic layers in such structures are coupled by nonlocal and, as a function of normal-metal layer thickness, an oscillatory (Parkin et al., 1990) exchange interaction that can be qualitatively understood by perturbation theory analogous to the RKKY (Ruderman and Kittel, 1954; Kasuya, 1956; Yosida, 1957) exchange coupling between magnetic impurities in a normal-metal host. Different oscillation periods, which can be resolved in measured magnetization configuration as a function of spacer thickness, are well explained in terms of the normalmetal Fermi-surface calipers in the growth direction. The magnetic ground-state configuration is, at least in principle, accessible to first-principles electronicstructure calculations in the spin-density-functional theory formalism, and that is basically the end of the story. However, in order to make a connection to the main topic of this review, we briefly discuss the formulation of the equilibrium exchange coupling in terms of scattering theory (Slonczewski, 1989, 1993; Erickson et al., 1993), which can also be formulated from first principles and calculated by density-functional theory (Bruno, 1995; Stiles, 1999). Another advantage of a scattering-theory formulation is that effects of disorder can be understood by employing the machinery of mesoscopic physics, such as random-matrix (Beenakker, 1997) or diagrammatic perturbation theory.

Let us consider a layered $N|F| N|F| N$ spin valve with angle $\theta$ between the magnetizations and an $N$ spacer with thickness $L$, see Fig. 1 schematic. Suppose we can view the $F|N| F$ trilayer as some spin-dependent scatterer embedded in a normal-metal medium. The trilayer gives a $\theta$-dependent contribution to the total ground- 


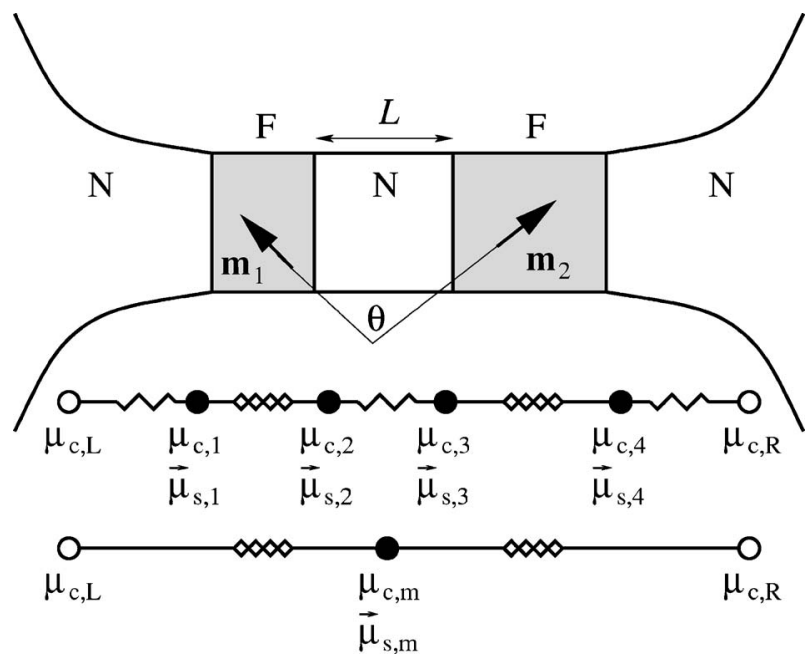

FIG. 1. Spin-valve schematic: two monodomain ferromagnets $(F)$ separated by a normal $(N)$ spacer and attached to normal leads. In this current perpendicular to the interface plane (CPP) configuration, the current flowing between two normal reservoirs sequentially traverses two magnetic layers. Also shown are two effective circuits discussed in Sec. II.A for the semiclassical regime where the interlayer exchange coupling vanishes.

state energy, given by a standard formula (Akkermans et al., 1991)

$$
E(L, \theta)=\frac{1}{2 \pi i} \int_{-\infty}^{\varepsilon_{F}} \varepsilon \frac{\partial}{\partial \varepsilon} \ln \operatorname{det} \mathbf{s}(L, \theta, \varepsilon) d \varepsilon,
$$

in terms of the energy-dependent scattering matrix $\mathbf{s}(L, \theta, \varepsilon)$ of the trilayer. The scattering matrix is made up from the matrices $\mathbf{r}$ and $\mathbf{t}$ of the reflection and transmission coefficients for a basis of spin-resolved incoming states at energy $\varepsilon$ from the right normal metal, whereas the primed ones ( $\mathbf{r}^{\prime}$ and $\left.\mathbf{t}^{\prime}\right)$ are defined for states coming from the left normal metal:

$$
\mathbf{s}=\left(\begin{array}{ll}
\mathbf{r} & \mathbf{t}^{\prime} \\
\mathbf{t} & \mathbf{r}^{\prime}
\end{array}\right)
$$

The scattering matrix of the total system can be composed out of the transmission and reflection coefficients of the $F \mid N$ interfaces as well as of the bulk layers by well-known concatenation rules. Quantum-well states and resonances are formed by multiple reflections at interfaces caused by potential steps and electronicstructure mismatches. The angle and thickness dependence of the total energy (1) can be understood in terms of the variation of the interference pattern of the spindependent electron waves in and close to the normalmetal spacer. By varying $L$ and $\theta$, quantum-well states enter or leave the Fermi sea with abrupt changes in the total energy that can be large for small $L$. The minimum energy for a given $L$ is usually found at $\theta=0$ and $\pi$, i.e., either parallel or antiparallel configurations are favored. Though exponentially suppressed, the coupling between the magnetic layers persists when the insertion is an insulating barrier (Slonczewski, 1989; Bruno, 1995).
Of special interest is the asymptotic dependence of energy $E(L, \theta)$ for large $L$. In this limit, the sharp jumps in energy by the population or depopulation of individual quantum-well states as a function of $L$ and $\theta$ become less prominent. The quasicontinuous angular dependence of the energy in this limit is well described by the lowest term in the expansion into Legendre polynomials:

$$
E(L, \theta) \stackrel{L \rightarrow \infty}{=} \cos \theta \sum_{\alpha} \frac{J_{\alpha}}{L^{2}} \sin \left(q_{\alpha \perp} L+\phi_{\alpha}\right),
$$

which is a sum of contributions from each critical caliper at the Fermi surface of the normal-metal spacer labeled by $\alpha$. The parameters $J_{\alpha}$ and $\phi_{\alpha}$ are model and material dependent (Stiles, 1999). $q_{\alpha \perp}$ is the critical Fermisurface-spanning wave vector in the layering direction, which determines the caliper in reciprocal space. Note that whereas we in principle require the scattering matrix for all occupied states in Eq. (1), Eq. (3) is governed by the scattering coefficients at the Fermi energy only, just as the transport properties at low temperature and bias. In practice, Eq. (3) can often be used for all but the most narrow spacer layers.

At configurations that are not at equilibrium, the derivative

$$
\tau=-\frac{\partial}{\partial \theta} E(L, \theta)
$$

does not vanish. A finite $\tau$ is therefore interpreted as an exchange torque acting on the magnetizations, pulling them into an energetically favorable configuration. Physically, this torque is an angular-momentum transfer that is carried by the electron spin. A spin valve that is "strained" by a relative misalignment of the magnetization directions from their lowest-energy configuration therefore supports dissipationless spin currents. The situation is quite analogous to the Josephson junction in which a difference of the superconducting phase over a weak link induces a supercurrent. We note that at finite temperatures, the ground-state energy $E(L, \theta)$ should be replaced with the free energy $F(L, \theta)$ in Eq. (4).

Essential for the existence and the magnitude of the nonlocal exchange coupling and the corresponding persistent spin currents is the phase coherence of the wave functions in the normal spacer. An incoming electron in the spacer with information of the left magnetization direction has to be reflected at the right interface and interfere with itself at the left interface in order to convey the coupling information. This implies strong sensitivity to the effects of impurities since the diffuse scattering destroys the regular interference pattern required by a sizable coupling. This qualitative notion has been formulated by Waintal et al. (2000) in the scatteringtheory formalism invoking the isotropy condition for validity of the random-matrix theory. Isotropy requires diffuse transport, viz., that $L$ is larger than the mean free path due to bulk and interface scattering. It can then be rigorously shown that the equilibrium spin currents vanish on average with fluctuations that scale like $N^{-1}$, 
where $N$ stands for the number of transverse transport channels in the normal-metal spacer. In layered metallic structures, $N$ is large and the static exchange coupling and spin currents can safely be disregarded in the diffuse limit. On top of the suppression by disorder, the magnitude of the coupling scales like $L^{-2}$ even in ballistic samples, see Eq. (3). Experimentally, even the presumably best $\mathrm{Co}|\mathrm{Cu}|$ Co samples indeed do not show an appreciable coupling beyond a spacer-layer thickness of about 20 atomic monolayers.

In Sec. $\mathrm{V}$ we discuss the magnetization dynamics of multilayers and superlattices. We find that, on top of the equilibrium spin currents that communicate the nonlocal static exchange coupling, a dynamic exchange interaction with a much longer range becomes important. Any significant coupling at equilibrium in dynamic studies can be represented approximately in terms of parametrized conservative forces that react to deviations from equilibrium without interference with nonequilibrium spin currents.

GMR was originally discovered in a configuration in which the current flows in the plane of the film. Multilayer pillar structures in which the current flows perpendicular to the planes (Pratt et al., 1991; Gijs et al., 1993; Gijs and Bauer, 1997) are more relevant in the present context. Assuming diffusive transport, the GMR with current flows perpendicular to the planes is easily understood in terms of a two-channel series-resistor model (Valet and Fert, 1993). In the parallel configuration, the charge current is short-circuited by the lowelectrical-resistance spin channel. The charge and spin transport in intermediate configurations in which magnetizations vary between parallel and antiparallel is important in the context of the present review due to the emergence of transverse spin currents that are absorbed by magnetizations and contribute as a driving torque to the dynamics. This dissipative channel for transverse spins also modifies the angular magnetoresistance of spin valves. These and other noncollinear magnetoelectronic dc phenomena are reviewed by Brataas, Bauer, and Kelly (2005).

\section{Landau-Lifshitz-Gilbert phenomenology}

At temperatures well below the ferromagnetic critical temperature $T_{c}$, the equilibrium magnetization of a bulk ferromagnet saturates at some material-specific value $M_{s}$. Symmetry-restoring Goldstone modes of this broken-symmetry state are spin waves (magnons) mentioned in Sec. I.A, which can condense into macroscopic transverse magnetization dynamics. In itinerant ferromagnets, there are also longitudinal spin excitations, the Stoner modes. However, since macroscopic variation of the magnetization magnitude at $T \ll T_{c}$ is very costly in free energy, we focus on the purely transverse motion of the position-dependent magnetization direction $\mathbf{m}$ $=\mathbf{M} / M_{s}$, with a fixed magnitude $|\mathbf{M}|=M_{s}$.

A traditional starting point in studying the transverse magnetization dynamics in a ferromagnetic medium is based on the phenomenological Landau-Lifshitz equation (Landau et al., 1980). The magnetization direction $\mathbf{m}(\mathbf{r}, t)$ is treated in this approach as a classical positionand time-dependent variable obeying equations of motion which are determined by the free-energy functional $F[\mathbf{M}]$ for degrees of freedom coupled to the magnetization distribution $\mathbf{M}(\mathbf{r})$ (such as the electromagnetic field or itinerant electrons experiencing a ferromagnetic exchange field):

$$
\partial_{t} \mathbf{m}(\mathbf{r}, t)=-\gamma \mathbf{m}(\mathbf{r}, t) \times \mathbf{H}_{\mathrm{eff}}(\mathbf{r}),
$$

where $\gamma$ is (minus) the gyromagnetic ratio and

$$
\mathbf{H}_{\mathrm{eff}}(\mathbf{r})=-\partial_{\mathbf{M}} F[\mathbf{M}]
$$

is the effective magnetic field. Corresponding to the respective contributions to the free energy, the effective field can usually be decomposed into the applied, dipolar demagnetization, crystal-anisotropy, and exchange fields. In the case of free electrons, $\gamma=2 \mu_{B} / \hbar>0$, in terms of Bohr magneton $\mu_{B}$ and Planck's constant $h$ $=2 \pi \hbar$, and it is usually close to this value in transitionmetal ferromagnets.

It is easy to see that the Landau-Lifshitz equation (5) with effective field (6) describes transverse magnetization dynamics preserving the free energy $F[\mathbf{M}]$. Definition (6) with the effective field depending on the instantaneous magnetic configuration assumes that the magnetization dynamics are very slow on the scale of the relevant microscopic relaxation processes. However, some slow degrees of freedom may not respond sufficiently fast to the magnetization motion, making the effective field dependent on the history of the magnetization dynamics $\mathbf{M}(\mathbf{r}, t)$. This should be associated with dissipation of energy into the degrees of freedom that are coupled to the magnetization.

As a specific example, consider the magnetization dynamics (5) described by the effective field

$$
\mathbf{H}_{\mathrm{eff}}(\mathbf{r}, t)=-\partial_{\mathbf{M}}\langle H(\mathbf{M})\rangle_{t},
$$

where $H(\mathbf{M})$ is the many-body Hamiltonian for itinerant electrons, parametrized by a mean-field magnetic configuration $\mathbf{M}(\mathbf{r}, t)$ of, e.g., some localized magnetic orbitals (as in the $s-d$ model), and \langle\rangle$_{t}$ evaluates its expectation value for the many-body state (or ensemble) at time $t$. Setting the many-body ensemble at time $t$ to its thermal equilibrium configuration determined by $\mathbf{M}(t)$ reproduces the Landau-Lifshitz definition (6). In the opposite extreme, when electrons do not respond at all to the fast magnetic dynamics, \langle\rangle$_{t} \approx\langle\rangle_{0}$, the effective field is determined by the history-independent functional $\langle H(\mathbf{M})\rangle_{0}$ instead. In the intermediate regime, a finite time lag in the response of the itinerant electrons to the varying magnetization causes dissipation of the magnetic energy, as discussed in Sec. VI.B. To lowest order in frequency (i.e., keeping only terms linear in $\partial_{t}$ ), such damping can be described by an additional torque in Eq. (5) (Gilbert, 1955, 2004): 


$$
\partial_{t} \mathbf{m}=-\gamma \mathbf{m} \times \mathbf{H}_{\mathrm{eff}}+\alpha \mathbf{m} \times \partial_{t} \mathbf{m},
$$

where $\alpha$ is the dimensionless Gilbert constant and $\mathbf{H}_{\text {eff }}$ is an effective field depending only on the instantaneous magnetic configuration. (Partial time derivatives imply here the possibility of spatial variations of the magnetization, as, e.g., in the case of spin waves; the full time derivatives are reserved for the monodomain dynamics.) The Gilbert term in Eq. (8) has been obtained for various microscopic formulations of the magnetization dynamics, see, e.g., Heinrich et al. (1967), Korenman and Prange (1972), Lutovinov and Reizer (1979), Safonov and Bertram (2000), Kunes and Kamberský (2002), Tserkovnyak et al. (2002a), and Sinova et al. (2004).

Energy dissipation implied by Eq. (8) preserves the local magnitude of the magnetization. For example, for a constant $\mathbf{H}_{\text {eff }}$ obeying Eq. (6) and $\alpha=0$, $\mathbf{m}$ precesses around the field vector with frequency $\omega=\gamma H_{\text {eff }}$. When damping is switched on, $\alpha>0$ (assuming positive $\gamma$, as in the case of free electrons), the precession spirals down on a time scale of $(\alpha \omega)^{-1}$ to a time-independent magnetization along the field direction, i.e., the lowest-freeenergy state. Close to an equilibrium axis with rotational symmetry, the Landau-Lifshitz-Gilbert equation (8) is obeyed by a small-angle damped circular precession, while in the presence of anisotropies, small-angle trajectories are elliptic and the damping is in general a tensor. For most of our purposes, simple circular precession with a scalar damping $\alpha$ suffices (but see Sec. VII.B). It is sometimes convenient to work with a different Gilbert parameter

$$
G=\alpha \gamma M_{s} .
$$

It can be made explicit that magnetization dynamics described by Eq. (8) dissipate energy at a rate determined by $\alpha$. To this end, suppose for simplicity that $\gamma \mathbf{H}_{\text {eff }}=\omega_{0} \hat{\mathbf{z}}, \omega_{0}>0$, is uniform throughout a monodomain ferromagnetic sample, so that Eq. (8) describes a damped macrospin circular precession around the $z$ axis. Small-angle dynamics around the $z$ axis can thus be resonantly excited by a (right-hand) circularly polarized rf field with a small amplitude $h_{\mathrm{rf}}$ and frequency $\omega$ close to $\omega_{0}$, that is, $h_{-}(t)=h_{x}(t)-i h_{y}(t)=h_{\mathrm{rf}} \exp (-i \omega t)$. The magnetic response to such a field is $\delta M_{-}(\omega)=\chi_{-+}(\omega) h_{-}(\omega)$, where

$$
\chi_{-+}(\omega)=\frac{\gamma M_{s}}{\left(\omega_{0}-\omega\right)-i \alpha \omega}
$$

is the transverse magnetic susceptibility. The linearresponse expression for the energy-dissipation power per unit of volume,

$$
P=\omega \operatorname{Im} \chi_{-+}(\omega) h_{\mathrm{rf}}^{2}=\frac{\alpha \gamma M_{s} \omega^{2} h_{\mathrm{rf}}^{2}}{\left(\omega_{0}-\omega\right)^{2}+(\alpha \omega)^{2}},
$$

does not depend on the microscopic origin of $\alpha$, as long as Eq. (8) holds. For a steady precession, one can also show that $P=-\mathbf{h}(t) \cdot \dot{\mathbf{m}}(t) M_{s}$ is the work done by the $\mathrm{rf}$ field $\mathbf{h}(t)$. The stability of the system, $P>0$, requires that $\alpha \gamma>0$.

Equation (8) has been found to successfully describe the dynamics of ultrathin ferromagnetic films as well as bulk materials in terms of a few material-specific parameters that are accessible to ferromagnetic-resonance (FMR) experiments (Bhagat and Lubitz, 1974; Heinrich and Cochran, 1993). FMR spectra are obtained by placing the sample into a microwave cavity and sweeping the external dc field. $\gamma \mathbf{H}_{\text {eff }}$ then determines the position and $\alpha$ the width of the resonance absorption peak. The FMR linewidth can have an additional contribution due to inhomogeneities in $\mathbf{H}_{\text {eff }}$, loosely corresponding to a finite range of the resonance frequency $\omega_{0}$ in Eq. (11). For example, small disorder by surface roughness or a nonuniform surface field in exchange-biased thin films contributes to the resonance broadening by (in quantummechanical terms) two-magnon scattering (Mills and Rezende, 2003). The inhomogeneous linewidth broadening is associated with dephasing of the global precession that in general does not conserve the magnitude of the magnetization. Whereas the Gilbert damping predicts a strictly linear dependence of FMR linewidths on frequency, the inhomogeneous broadening is usually associated with weaker frequency dependence as well as a zero-frequency contribution. Another common technique in studying long-wavelength spin waves is Brillouin light scattering (see, e.g., Demokritov and Tsymbal, 1994). Both FMR and Brillouin light scattering probe magnetic excitations close to the surface, i.e., within the corresponding skin depth of the order of $100 \mathrm{~nm}$ for FMR and $10 \mathrm{~nm}$ for Brillouin light scattering (Mills and Rezende, 2003). In contrast to FMR, Brillouin light scattering excites spin waves with finite wavelengths in the surface plane (in the range of visible light), bearing consequences for the signal linewidths; see Mills and Rezende (2003) and Sec. VII.C. In closing this subsection, we remark that ferromagnetic magnetization dynamics and, in particular, magnetization relaxation processes are collective many-body phenomena that continue to fascinate in spite of decades of theoretical and experimental efforts to understand them; see, e.g., Qian and Vignale (2002); Dobin and Victora (2003).

\section{Current-induced magnetization dynamics}

It has been only relatively recently realized that in magnetic multilayers the magnets can be excited by other means than external magnetic fields. Berger (1996) and Slonczewski (1996) predicted that in current perpendicular to the interface plane spin-valve structures a dc current in the right direction can excite and even reverse the magnetization of a magnetic layer. This can be observed by monitoring $d I / d V$, which depends on the magnetic configuration, as in GMR. The predictions have now been amply confirmed by many recent experiments (Myers et al., 1999, 2002; Katine et al., 2000; Tsoi et al., 2000; Wegrowe et al., 2000; Ji et al., 2003; Kiselev et al., 2003; Özyilmaz et al., 2003; Urazhdin et al., 2003; Pufall 
et al., 2004; Krivorotov et al., 2005; Sun et al., 2005). Current-induced magnetic dynamics have also been found to affect current noise spectra (Covington et al., 2004). The prediction (Polianski and Brouwer, 2004; Stiles et al., 2004) that even a single dc-current-driven ferromagnetic layer may undergo a resonant finitewave-vector spin-wave excitation has been experimentally confirmed by Özyilmaz et al. (2004); see also Ji et al. (2003). Consequently, higher-wave-vector spin-wave excitations may in some situations successfully compete (Brataas, Tserkovnyak, and Bauer, 2005; Özyilmaz et al., 2005) with current-induced macrospin motion considered below. Current-induced magnetization dynamics pose a challenging physics problem that requires understanding of the coupling of nonequilibrium quasiparticles with the collective magnetization dynamics. It carries technological potential as well. In small structures, to write information into magnetic RAM's, switching by Ampère magnetic fields may need more power than switching by current-induced magnetization reversal. The generation of microwaves by exciting stable magnetization orbits with dc bias-dependent frequencies may also satisfy technological needs (Kiselev et al., 2003; Rippard et al., 2004).

Current-induced magnetization dynamics are a consequence of spin-dependent transport in $F \mid N$ heterostructures. For example, Slonczewski's magnetization torque (Slonczewski, 1996) is equivalent to absorption of an incident spin current with a polarization component perpendicular to the magnetization (Brataas et al., 2000; Waintal et al., 2000; Stiles and Zangwill, 2002). The component of the electron spin perpendicular to the magnetization is not a constant of the motion in a ferromagnet. On the other hand, if one neglects the effects of spinorbit coupling (other than the macroscopic anisotropy already included in $\mathbf{H}_{\text {eff }}$ ) and other spin-flip processes, the total spin angular momentum is conserved. The spin angular-momentum difference between an electron entering and leaving a ferromagnet is therefore transferred to the magnetization. Under a sufficiently large angularmomentum transfer, the magnetization starts to move. The component of the net spin angular-momentum flow out of the ferromagnet $\mathbf{I}_{s}$ parallel to $\mathbf{m}$ vanishes, since the outflow cancels the inflow for the parallel component (assuming spin along the uniform magnetization direction is conserved). The spin-transfer torque $\boldsymbol{\tau}=-\mathbf{I}_{s}$ should be accounted for as an additional source term in the equation motion of the magnetization. In the presence of spin-flip scattering, a component parallel to $\mathbf{m}$ must be projected out to represent the torque that drives the transverse magnetization dynamics:

$$
\boldsymbol{\tau}=-\mathbf{m} \times \mathbf{I}_{s} \times \mathbf{m} .
$$

An electron injected into a ferromagnet at the Fermi energy and transverse polarization is not in a momentum eigenstate, but should be described by a linear combination of majority and minority spin eigenstates associated with different Fermi wave vectors, $k_{F}^{\uparrow}$ and $k_{F}^{\downarrow}$. The linear coefficients of up and down spins carry out oscillations as a function of time and position, equivalent to a spin precession around the exchange magnetic field. Fermi-level states entering the ferromagnet at different angles precess on different length scales perpendicular to the interface, depending on the perpendicular component of the spin-up and spin-down wave-vector difference. In ferromagnets with a large cross-section area, a large number of transverse modes with different spinprecession lengths contribute to the total spin current. The destructive interference of numerous states with different phases corresponds to the absorption of the transverse spin current inside the ferromagnet on the scale of the transverse-spin coherence length,

$$
\lambda_{\mathrm{sc}}=\frac{\pi}{\left|k_{F}^{\uparrow}-k_{F}^{\downarrow}\right|} .
$$

$\lambda_{\mathrm{sc}} \sim \lambda_{F}$ (the Fermi wavelength), an atomistic length scale for, e.g., transition-metal ferromagnets like $\mathrm{Co}, \mathrm{Ni}$, and $\mathrm{Fe}$, or their alloys. The smallness of penetration depths $\lambda_{\mathrm{sc}}$ a posteriori justifies the implicitly assumed clean limit, $\lambda_{\text {sc }} \ll \lambda$ (the mean free path). It should be noted that $\lambda_{\mathrm{sc}}$ sets a length scale of a power law (Stiles and Zangwill, 2002), not an exponential suppression of the transverse spin current.

After transmission through a ferromagnetic film much thicker than $\lambda_{\mathrm{sc}}$, electrons are completely polarized along the magnetization direction. When reflection at the $F \mid N$ boundary may be disregarded, $\mathbf{I}_{s}$ on the righthand side of Eq. (12) is simply the negative of the transverse spin current incident on the ferromagnet. When reflection cannot be neglected, the transverse polarization of the reflected electrons should be taken into account. Although reflected electrons hardly penetrate the ferromagnet (over the Fermi wavelength), the strong exchange field can still induce a significant precession of the reflected component (Stiles and Zangwill, 2002). This can lead to a reaction torque on the ferromagnet as an effective magnetic field oriented parallel to the spin accumulation in the normal metal. However, at interfaces to transition-metal ferromagnets, positive and negative contributions to the effective field typically average out to be small (Xia et al., 2002).

The dynamics of a monodomain ferromagnet of volume $V$ and magnetization $M_{s}$ that is subject to the torque (12) are modified by an additional source term on the right-hand side of the Landau-Lifshitz-Gilbert equation (Slonczewski, 1996):

$$
\left.\frac{\partial \mathbf{m}}{\partial t}\right|_{\text {torque }}=\frac{\gamma}{M_{s} V} \mathbf{m} \times \mathbf{I}_{s} \times \mathbf{m} .
$$

For a fixed current density, Eq. (14) is proportional to the interface area and inversely proportional to the volume of the ferromagnet. Current-induced magnetization dynamics are usually realized in perpendicular spin valves with one hard (highly coercive) ferromagnet that acts as a static polarizer and a second soft ferromagnet that responds sensitively to the spin-transfer torque. 


\section{E. Spin emission by excited ferromagnets}

When seeking a consistent theory of magnetization dynamics in heterostructures, the current-induced magnetization torque discussed above is only one side of the coin. A moving magnetization in a ferromagnet that is in electric contact with normal conductors emits ("pumps") a spin current into its nonmagnetic environment (Tserkovnyak et al., 2002a), giving a contribution to $\mathbf{I}_{s}$ in Eq. (14). The spin pumping thus leads to an additional source term in the Landau-Lifshitz-Gilbert equation even when the magnetization dynamics are induced by external magnetic fields and not by applied current bias. In typical biased systems with current-induced dynamics, the spin pumping is of the same order as the currentdriven torque and should be treated on an equal footing, as explained in Sec. V.C.

Spin pumping by a precessing ferromagnet is, in some sense, the reverse process of current-induced magnetization dynamics. When the pumped spin angular momentum is not quickly dissipated to the normal-metal atomic lattice, a spin accumulation builds up and creates reaction torques due to transverse-spin backflow into ferromagnets. The interplay between magnetization dynamics and the nonequilibrium spin-polarized transport in heterostructures is the central topic of this review. The conversion of magnetization movement into spin currents and vice versa at a possibly different location is what we mean by the nonlocality of the magnetization dynamics in our title. In the remainder of this subsection, we put this topic into a historic perspective.

Nonlocality of the magnetization dynamics can be interpreted as a nonlocal exchange coupling with explicit time dependence. A first step in this direction was carried out by Barnes (1974), who generalized the RKKY theory for the static linear response of the electron gas to magnetic impurities to dynamic phenomena in order to understand the electron-spin resonance of localized magnetic moments embedded in a conducting medium. He showed that the dynamic part of the RKKY interaction in diffuse media is limited by the spin-diffusion length. A related experimental observation of "giant electron-spin-resonance transmission" through a $\mathrm{Cu}$ foil implanted with magnetic Mn ions on one or both sides (Monod et al., 1972) showed that precessing impurity magnetic moments cause nonequilibrium spin diffusion.

Subsequently, Silsbee et al. (1979) observed a strong enhancement of the microwave transmission through a $\mathrm{Cu}$ foil with a thin ferromagnetic layer evaporated on one side, when the ferromagnetic and $\mathrm{Cu}$ conductionelectron-spin resonances are tuned into a collective mode. This is related to the enhancement of Larmor waves in nonresonant electron-spin transmission through normal-metal foils coated with a ferromagnetic layer (Janossy and Monod, 1976). The experiments were interpreted by postulating a phenomenological spin interdiffusion through the $F \mid N$ interface by nonequilibrium components of the magnetization or spin accumulation on both sides. These authors concluded that the precessing magnetic moments can be a source of non- equilibrium spin accumulation diffusing through the nonmagnetic conducting medium. Vice versa, the nonequilibrium spin accumulation can be transferred into the magnetization motion. This picture was investigated further by Janossy (1980) and Parks and Silsbee (1987) and was invoked later to qualitatively interpret the experiments by Hurdequint and Dunifer (1988), Hurdequint (1991), and Hurdequint and Malouche (1991).

The discussion of the dynamic coupling between a precessing magnetization and itinerant electrons in layered $F \mid N$ structures was (independently) revived by Berger (1996). He predicted an enhanced Gilbert damping in thin ferromagnetic films in trilayer $F|N| F$ configurations, relying on an elementary quantum process of magnon annihilation associated with electron spin flip. A very different approach to the problem was considered by Tserkovnyak et al. (2002a). They used the formalism of parametric pumping (Büttiker et al., 1994; Brouwer, 1998) developed in the context of mesoscopic scattering problems in order to show that the timedependent magnetization induces spin emission into the itinerant degrees of freedom; see Sec. III. [A host of other mesoscopic spin pumps have been proposed in recent years; see, e.g., Sharma and Brouwer (2003) and references therein, at least one of which has been realized experimentally (Watson et al., 2003).] The spinpumping picture enables us to discuss several topics of this review in a unified manner and is easily rendered quantitatively. More recently, a linear-response formalism similar to that of Barnes (1974) has been put forward by Šimánek and Heinrich (2003). This alternative point of view has the advantage of being more familiar to many in the magnetism community, but it is much less suited for quantitative comparison with experiments, as discussed in Sec. VI.

\section{SCATTERING-THEORY APPROACH TO MAGNETOELECTRONICS}

\section{A. Magnetoelectronic dc circuit theory}

Electron spin and charge transport in $F \mid N$ heterostructures with static magnetic configurations has attracted considerable attention since the discovery of GMR. Most of the activity in recent years, including the work reviewed here, has concentrated on the current perpendicular to the interface plane geometry in which the electrons pass sequentially through magnetic and nonmagnetic elements of the circuit. See Gijs and Bauer (1997) for a review. A systematic and quite general, yet easy-to-handle, semiclassical approach to this problemthe magnetoelectronic circuit theory-is reviewed by Brataas, Bauer, and Kelly (2005). In the following we give a brief account of that theory before extending it to dynamic magnetic configurations in Sec. III.

A basic element of the magnetoelectronic circuit theory is a magnetic "interconnector" between two normal nodes or reservoirs attached to the ferromagnet via Ohmic leads, as shown in Fig. 2. Physically, this could be realized, e.g., as a layered pillar $N|F| N$ structure. The 


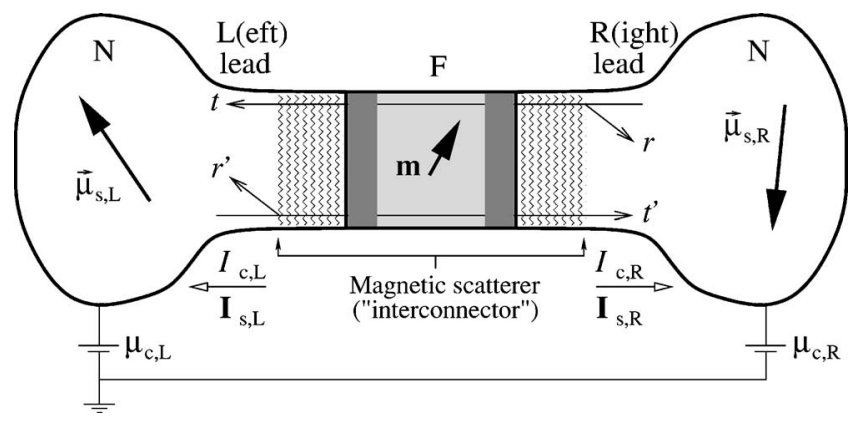

FIG. 2. Magnetic scatterer (interconnector) connecting two chaotic normal-metal $(N)$ nodes via ballistic leads that support a quantized number of transverse channels at the Fermi energy. The scatterer includes a ferromagnetic $(F)$ region characterized by a uniform magnetic direction $\mathbf{m}$ (but not necessarily uniform magnitude of the exchange spin splitting along $\mathbf{m}$ ), which is depicted as the gray box in the center. The dark-gray areas of the ferromagnetic region near both $F \mid N$ interfaces mark the extent of the transverse spin coherence characterized by $\lambda_{\mathrm{sc}}$, Eq. (13). Each of the two normal-metal regions is divided into a reservoir characterized by the electrochemical potential $\mu_{c}$ and (vector) spin accumulation $\boldsymbol{\mu}_{s}$, a ballistic lead with a fixed number of transport channels, and possibly a disordered region incorporated in the interconnector (depicted by wavy lines), which accounts for relevant normal-metal scattering processes. The interconnector is described by spindependent reflection and transmission coefficients forming the scattering matrix, Eq. (21), for quantum channels in the normal leads. The purpose of the formalism is to calculate the nonequilibrium charge and spin flows in the leads, $I_{c}$ and $\mathbf{I}_{s}$, induced by spin accumulations and electrochemical-potential imbalance in the nodes.

normal nodes are chaotic to the extent that the nonequilibrium transport through the leads can be expressed in terms of energy-dependent distribution functions $\hat{f}(\varepsilon)$ in each node (averaged over orbital states at energy $\varepsilon$ ), which are $2 \times 2$ energy-dependent matrices in spin space of spin-1/2 electrons. (We make a convention of denoting such $2 \times 2$ matrices in spin space by hats.) In thermal equilibrium $\hat{f}(\varepsilon)=f_{\mathrm{FD}}(\varepsilon) \hat{\sigma}_{0}$, where $f_{\mathrm{FD}}(\varepsilon)$ is the FermiDirac distribution for electrons and $\hat{\sigma}_{0}$ is the $2 \times 2$ unit matrix. In linear response, it is convenient to define local electrochemical potentials $\mu_{c}$ and spin accumulations $\boldsymbol{\mu}_{s}$ in all nodes (Brataas et al., 2000, 2001):

$$
\begin{aligned}
& \mu_{c}=\frac{1}{2} \int_{\varepsilon_{0}}^{\infty} d \varepsilon \operatorname{Tr}[\hat{f}(\varepsilon)], \\
& \boldsymbol{\mu}_{s}=\int_{\varepsilon_{0}}^{\infty} d \varepsilon \operatorname{Tr}[\hat{\boldsymbol{\sigma}} \hat{f}(\varepsilon)],
\end{aligned}
$$

choosing a reference energy $\varepsilon_{0}$ that lies below the Fermi energy by much more than the thermal energy and voltage biases, but which is arbitrary otherwise. $\hat{\boldsymbol{\sigma}}$ $=\left(\hat{\sigma}_{x}, \hat{\sigma}_{y}, \hat{\sigma}_{z}\right)$ is a vector of the Pauli matrices. Notice that in our convention, the spin accumulation $\boldsymbol{\mu}_{s}$ is a vector with the direction determined by the total nonequilibrium spin-imbalance density $\mathbf{s}$ and the magnitude given by the corresponding energy splitting of spins along this direction. In linear response and at low temperatures, these quantities are related through the Fermi-level density of states (per spin and unit volume) in the node, $\mathcal{N}\left(\varepsilon_{F}\right): \mathbf{s}=(\hbar / 2) \mathcal{N}\left(\varepsilon_{F}\right) \boldsymbol{\mu}_{s}$.

Here we calculate the dc charge and spin angularmomentum currents, $I_{c}$ and $\mathbf{I}_{s}$, entering the nodes through the leads, which are induced by the nonequilibrium spin accumulations in the nodes and/or electrochemical imbalance between the nodes. It is convenient to define the $2 \times 2$ tensor current

$$
\hat{I}=\frac{1}{2} \hat{\sigma}_{0} I_{c}-\frac{e}{\hbar} \hat{\boldsymbol{\sigma}} \cdot \mathbf{I}_{s},
$$

whose isotropic and traceless components determine, respectively, the charge and spin currents. Since, as discussed above, spin currents are not conserved at $F \mid N$ interfaces, we use the convention that it is calculated on the normal side (unless specified otherwise). The $2 \times 2$ current operator $\hat{I}_{l}$ for the $l$ th lead $(l=L, R)$ can be expressed in terms of operators $a_{n, l}^{\sigma}(\varepsilon)\left[b_{n, l}^{\sigma}(\varepsilon)\right]$ that annihilate a spin- $\sigma$ electron with energy $\varepsilon$ leaving (entering) the $l$ th node through the $n$th quantum channel of the lead (Tserkovnyak and Brataas, 2001):

$I_{l}^{\sigma \sigma^{\prime}}=\frac{e}{h} \sum_{m} \int d \varepsilon d \varepsilon^{\prime}\left[a_{m, l}^{\sigma^{\prime}}(\varepsilon)^{\dagger} a_{m, l}^{\sigma}\left(\varepsilon^{\prime}\right)-b_{m, l}^{\sigma^{\prime}}(\varepsilon)^{\dagger} b_{m, l}^{\sigma}\left(\varepsilon^{\prime}\right)\right]$.

Suppose, as a starting point, that the momentum-space distribution in each node is isotropic, i.e., $\hat{f}(\varepsilon)$ does not depend on orbital quantum numbers. This is true if the nonequilibrium currents do not cause significant drift contributions to the distribution function. (We shall revisit and drop this assumption in Sec. II.B.) For the $l$ th lead then

$$
\left\langle a_{n, l}^{\sigma}(\varepsilon)^{\dagger} a_{n^{\prime}, l^{\prime}}^{\sigma^{\prime}}\left(\varepsilon^{\prime}\right)\right\rangle=f_{l}^{\sigma^{\prime} \sigma}(\varepsilon) \delta_{l l^{\prime}} \delta_{n n^{\prime}} \delta\left(\varepsilon-\varepsilon^{\prime}\right),
$$

and it is now straightforward to evaluate the expectation value $\left\langle I_{l}^{\sigma \sigma^{\prime}}\right\rangle$ (also denoted simply by $I_{l}^{\sigma \sigma^{\prime}}$ ) of the current operator after relating the scattered states to the incoming states via the scattering matrix of the magnetic interconnector:

$$
b_{n, l}^{\sigma}(\varepsilon)=\sum_{\sigma^{\prime} n^{\prime}, l^{\prime}} s_{n n^{\prime}, l l^{\prime}}^{\sigma \sigma^{\prime}}(\varepsilon) a_{n^{\prime}, l^{\prime}}^{\sigma^{\prime}}(\varepsilon) .
$$

The scattering coefficients $s_{n n^{\prime}, l l^{\prime}}^{\sigma \sigma^{\prime}}$ characterize reflection if $l=l^{\prime}$ and transmission otherwise. Equation (20) assumes that the entire interconnector is elastic, so that the electron energy is conserved upon scattering between the normal nodes. For a ferromagnet with an exchang spin splitting along unit vector $\mathbf{m}$ and vanishing spin-orbit interaction in the system (Brataas et al., 2000),

$$
\hat{s}_{n n^{\prime}, l l^{\prime}}=s_{n n^{\prime}, l l^{\prime}}^{\uparrow} \hat{u}^{\uparrow}+s_{n n^{\prime}, l l^{\prime}}^{\downarrow} \hat{u}^{\downarrow}
$$

in terms of the scattering coefficients for spins up (down) along $\mathbf{m}, s_{n n^{\prime}, l l^{\prime}}^{\uparrow(\downarrow)}$ and the projection matrices 
TABLE I. Calculated interface conductances (in units of quantum channels per $\mathrm{nm}^{2}$ ). The results are shown for clean and disordered interfaces. The latter are modeled by two atomic monolayers of $50 \%$ alloy. From Zwierzycki et al., 2005.

\begin{tabular}{ccccccccc}
\hline \hline System & Interface & $g^{\uparrow \uparrow}$ & $g^{\downarrow \downarrow}$ & $g_{r}^{\uparrow \downarrow}$ & $g_{i}^{\uparrow \downarrow}$ & $g_{N}^{\text {Sh }}$ & $g_{F \uparrow}^{\text {Sh }}$ & $g_{F \downarrow}^{\text {Sh }}$ \\
\hline $\mathrm{Au}_{\mathrm{fcc}} \mid \mathrm{Fe}_{\mathrm{bcc}}$ & clean & 10.3 & 2.1 & 12.0 & 0.1 & 11.9 & 21.4 & 11.9 \\
$(001)$ & alloy & 10.1 & 4.6 & 11.9 & 0.1 & & & \\
$\mathrm{Cu}_{\mathrm{fcc}} \mid \mathrm{Co}_{\mathrm{fcc}}$ & clean & 10.8 & 9.8 & 14.1 & 0.4 & 15.0 & 11.9 & 27.9 \\
$(111)$ & alloy & 10.8 & 8.5 & 14.6 & -1.1 & & & \\
\hline \hline
\end{tabular}

$$
\hat{u}^{\uparrow(\downarrow)}=\frac{1}{2}\left(\hat{\sigma}_{0} \pm \hat{\boldsymbol{\sigma}} \cdot \mathbf{m}\right)
$$

A consequence of the elastic-scattering approximation is a rigid exchange field; there are no magnons excited by the electron transport. If we, furthermore, assume a sufficiently low temperature, voltage imbalance, and spin accumulations, so that the scattering-matrix variation on these energy scales is negligible, we can replace $\hat{s}_{n n^{\prime}, l l^{\prime}}(\varepsilon)$ by its value at the Fermi level $\varepsilon_{F}$.

It is then convenient to group the conductance parameters into two pairs of $2 \times 2$ matrices. For electrons incident from the right lead, we define

$$
\begin{aligned}
& g^{\sigma \sigma^{\prime}}=\sum_{n n^{\prime}}\left[\delta_{n n^{\prime}}-r_{n n^{\prime}}^{\sigma}\left(r_{n n^{\prime}}^{\sigma^{\prime}}\right)^{*}\right], \\
& t^{\sigma \sigma^{\prime}}=\sum_{n n^{\prime}} t_{n n^{\prime}}^{\sigma}\left(t_{n n^{\prime}}^{\sigma^{\prime}}\right)^{*},
\end{aligned}
$$

where the index $n^{\prime}$ is summed over the channels in the right lead, and $n$ runs over the channels in the right lead in Eq. (23) and left lead in Eq. (24). The coefficients $r_{n n^{\prime}}^{\sigma}$ and $t_{n n^{\prime}}^{\sigma}$ are reflection and transmission amplitudes, i.e., elements $s_{n n^{\prime}, l l^{\prime}}^{\sigma}$ of the scattering matrix (21) with $l \neq l^{\prime}$ and $l=l^{\prime}$, respectively. The range of summation for $n^{\prime}$ in Eqs. (23) and (24), i.e., the total number of transverse quantum channels in the right lead (for a given spin species at the Fermi level) is called the Sharvin conductance $g^{\mathrm{Sh}}$, a quantity which will be useful later. For electrons incident from the left lead, we denote the reflection and transmission amplitudes by primed quantities, and we similarly define primed matrices $g^{\prime \sigma \sigma^{\prime}}$ and $t^{\prime \sigma \sigma^{\prime}}$ in terms of the primed scattering amplitudes. We denote the Sharvin conductance of the left lead by $g^{\prime \text { Sh }}$. It should be understood that all scattering coefficients and corresponding conductance parameters are evaluated at the Fermi level. Putting the above equations together (Brataas et al., 2000, 2001, 2003),

$$
\begin{aligned}
I_{c, R}^{(0)}= & \frac{e}{2 h}\left\{2\left(g^{\uparrow \uparrow}+g^{\downarrow \downarrow}\right)\left(\mu_{c, R}-\mu_{c, L}\right)+\left(g^{\uparrow \uparrow}-g^{\downarrow \downarrow}\right)\right. \\
& \left.\times\left(\boldsymbol{\mu}_{s, R}-\boldsymbol{\mu}_{s, L}\right) \cdot \mathbf{m}\right\},
\end{aligned}
$$

$$
\begin{aligned}
\mathbf{I}_{s, R}^{(0)}= & -\frac{1}{8 \pi}\left\{2\left(g^{\uparrow \uparrow}-g^{\downarrow \downarrow}\right)\left(\mu_{c, R}-\mu_{c, L}\right) \mathbf{m}+\left(g^{\uparrow \uparrow}+g^{\downarrow \downarrow}\right)\right. \\
& \times\left[\left(\boldsymbol{\mu}_{s, R}-\boldsymbol{\mu}_{s, L}\right) \cdot \mathbf{m}\right] \mathbf{m}+2 g_{r}^{\uparrow \downarrow} \mathbf{m} \times \boldsymbol{\mu}_{s, R} \times \mathbf{m} \\
& +2 g_{i}^{\uparrow \downarrow} \boldsymbol{\mu}_{s, R} \times \mathbf{m}-2 t_{r}^{\uparrow \downarrow} \mathbf{m} \times \boldsymbol{\mu}_{s, L} \times \mathbf{m} \\
& \left.-2 t_{i}^{\prime \uparrow \downarrow} \boldsymbol{\mu}_{s, L} \times \mathbf{m}\right\},
\end{aligned}
$$

and the currents through the left lead are obtained by interchanging $L$ with $R, g^{\sigma \sigma^{\prime}}$ with $g^{\prime \sigma \sigma^{\prime}}$, and $t^{\prime \uparrow \downarrow}$ with $t^{\uparrow \downarrow}$. [The superscript (0) introduced here denotes currents with static magnetizations.] By unitarity of the scattering matrix, $g^{\sigma \sigma}=g^{\prime \sigma \sigma}=t^{\sigma \sigma}=t^{\prime \sigma \sigma}$ if the spin component along the magnetization direction is conserved. The dc transport in the two-terminal geometry is then determined by two real-valued spin-dependent conductances $g^{\sigma \sigma}$ and four complex-valued (spin-)mixing parameters $g^{\uparrow \downarrow}=g_{r}^{\uparrow \downarrow}$ $+i g_{i}^{\uparrow \downarrow}, t^{\uparrow \downarrow}=t_{r}^{\uparrow \downarrow}+i t_{i}^{\uparrow \downarrow}$ (the subscripts $r$ and $i$, respectively, denoting the real and imaginary parts), $g^{\prime \uparrow \downarrow}$, and $t^{\prime \uparrow \downarrow}$. For a mirror-symmetric structure, $g^{\uparrow \downarrow}=g^{\prime \uparrow \downarrow}$ and $t^{\uparrow \downarrow}=t^{\prime \uparrow \downarrow}$.

We now also have access to microscopic expressions for the spin-transfer torques (12). The torque on the right surface of the ferromagnet $\boldsymbol{\tau}_{R}=-\mathbf{m} \times \mathbf{I}_{s, R}^{(0)} \times \mathbf{m}$,

$$
\begin{aligned}
\boldsymbol{\tau}_{R}= & \frac{1}{4 \pi}\left(g_{r}^{\uparrow \downarrow} \mathbf{m} \times \boldsymbol{\mu}_{s, R} \times \mathbf{m}+g_{i}^{\uparrow \downarrow} \boldsymbol{\mu}_{s, R} \times \mathbf{m}-t_{r}^{\prime \uparrow \downarrow} \mathbf{m}\right. \\
& \left.\times \boldsymbol{\mu}_{s, L} \times \mathbf{m}-t_{i}^{\prime \uparrow \downarrow} \boldsymbol{\mu}_{s, L} \times \mathbf{m}\right),
\end{aligned}
$$

is proportional to the spin-mixing (i.e., off-diagonal) components of the conductance matrices (23) and (24). The first two terms in $\boldsymbol{\tau}_{R}$ involve reflection at the right $F \mid N$ junction and the last two terms transmission through the entire $N|F| N$ structure. The latter terms can thus be disregarded when the ferromagnet is much thicker than the transverse-spin coherence length $\lambda_{\mathrm{sc}}$, Eq. (13), since transmitted electrons accumulate phases differing by more than $\pi$ for opposite spins (along m). In that limit, the first term, proportional to $\mathbf{m} \times \boldsymbol{\mu}_{s, R} \times \mathbf{m}$, is similar to the torque introduced by Slonczewski (1996) that is responsible for instability leading to magnetization precession or reversal. The second term, proportional to $\boldsymbol{\mu}_{s, R} \times \mathbf{m}$, acts as an effective magnetic field collinear with spin accumulation in the right normal node. In transition-metal ferromagnets, $g_{i}^{\uparrow \downarrow} \lesssim 0.1 g_{r}^{\uparrow \downarrow}$, see, e.g., Table I and Sec. VII.A.1, so 


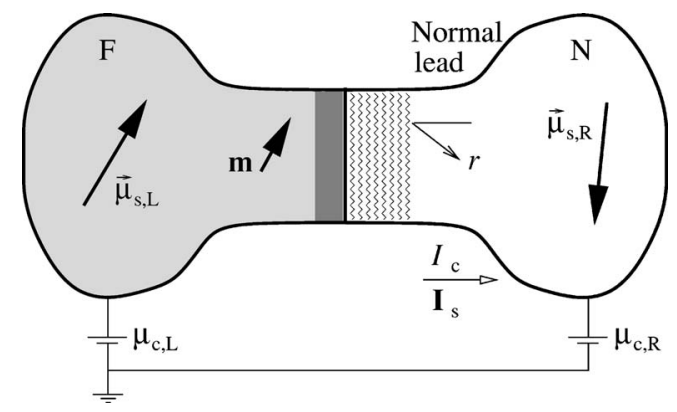

FIG. 3. Contact between a ferromagnetic and a normal node. The notation is analogous to Fig. 2. Here the charge and spin currents in the normal lead depend on the conductance matrix $g^{\sigma \sigma^{\prime}}$ defined in terms of the spin-dependent reflection coefficients from the normal-metal side, as before; see Eq. (23). The nonequilibrium spin accumulation in the ferromagnetic node is collinear with the magnetization $\mathbf{m}$.

that the effective magnetic field can be disregarded in many practical situations.

For ferromagnetic films much thicker than $\lambda_{\mathrm{sc}}$, the remaining mixing conductances $g^{\uparrow \downarrow}$ and $g^{\prime \uparrow \downarrow}$ are insensitive to scattering processes deep inside the ferromagnet (i.e., in the light-gray area of the ferromagnet in Fig. 2) and are determined by the scattering potential of a thin slice of the ferromagnet near the interfaces (the dark-gray areas) and eventually the normal metal (the wavy-line area). The mixing conductance is then a property of the isolated $F \mid N$ junction rather than the entire $N|F| N$ scatterer. In this limit, we introduce a ferromagnetic node at a sufficient distance from the interfaces and consider, without lack of generality, the two interfaces separately. This is allowed since in the bulk of the ferromagnet spin accumulation becomes a well-defined semiclassical distribution function collinear with the magnetization. An analysis entirely analogous to the one leading to Eqs. (25) and (26) results in the same equations for the currents $I_{c}$ and $\mathbf{I}_{s}$ (on the normal-metal side of the right interface). Only the terms proportional to $t_{r}^{\prime \uparrow \downarrow}$ and $t_{i}^{\prime \uparrow \downarrow}$ in Eq. (26) drop out now because of the collinearity of $\boldsymbol{\mu}_{\mathbf{s}, \mathbf{L}}$ and $\mathbf{m}$, the left node now being assumed ferromagnetic; see Fig. 3 (Brataas et al., 2000, 2001). Naturally, when the $F$-layer thickness is much larger than $\lambda_{\mathrm{sc}}$, the mixing conductance for the normal lead $g^{\uparrow \downarrow}$ in the $F \mid N$ structure of Fig. 3 is the same as $g^{\uparrow \downarrow}$ for the right lead in Fig. 2 (assuming the same $F \mid N$ contacts, including dark-gray and wavy-line areas at the right lead). On the other hand, the spin-up and spin-down conductances of the $N|F| N$ structure are not identical to the conductance parameters of a single $F \mid N$ interface, being dependent on two $F \mid N$ junctions and the bulk $F$ layer.

So far, we have focused on the spin and charge flow through a single resistive element, i.e., a single $N|F| N$ or $F \mid N$ junction. Equations (25) and (26), which can be viewed as a generalization of the Landauer-Büttiker formulas (see, e.g., Imry, 1997), describe charge and spin currents in a two-terminal geometry with possibly nonequilibrium spin accumulations in the nodes. These are basic building blocks of the magnetoelectronic circuit theory. We also need the Kirchhoff laws generalized in order to consider the spin currents and spin accumulations on an equal footing with the usual charge currents and voltage biases (Brataas, Bauer, and Kelly, 2005). The properties of a given device or circuit can be calculated by first prudently separating it into reservoirs, nodes, and resistors (interconnectors), where the latter are the current-limiting elements. The nodes are supposed to have a negligibly small resistance and their choice may depend on the problem at hand. In a disordered multilayer, for example, it is convenient to imagine (fictitious) nodes at both sides of an interface, treating the latter as a separate resistive element (whose conductance parameters may need to be redefined, however, as explained in Sec. II.B). Reservoirs represent the battery poles, i.e., large thermodynamic baths at thermal equilibrium with a constant electrochemical potential. The electrochemical potentials and spin accumulations in the nodes are not known a priori, but are determined from the generalized Kirchhoff laws based on the conservation of spin and charge. For example, disregarding spinflip scattering, in the stationary dc state, all spin and charge flows into a normal-metal node must vanish:

$$
\sum_{l} I_{c, l}=0, \quad \sum_{l} \mathbf{I}_{s, l}=0
$$

summing over all leads attached to the node. The net spin flow into a ferromagnetic node projected onto its magnetization also vanishes, whereas the transverse currents are absorbed at the interface, as discussed above. We have also seen that the spin and charge currents in each lead can be calculated as a function of the distributions on adjacent nodes and reservoirs in terms of welldefined conductance parameters. The spin- and chargecurrent conservation laws (28) then allow computation of the circuit properties as a function of, e.g., the voltages applied to the reservoirs. The protocol for calculating the current-voltage curves may be summarized as follows:

(1) Divide the circuit into resistors, nodes, and reservoirs.

(2) Specify the control variables, e.g., the voltages (electrochemical potentials) applied to the reservoirs. Parametrize the electrochemical potential and spin accumulation of each node.

(3) Compute the currents through the resistors as a function of the distributions in the adjacent nodes, which requires spin-dependent and spin-mixing conductances defined earlier.

(4) Use spin- and charge-current conservation laws (28) at each node. If there is spin decoherence, use a modified continuity equation with spin relaxation.

(5) Solve the resulting system of linear equations to obtain all currents as a function of electrochemical potentials of the reservoirs. 
The charge and spin currents through the resistors, the net spin torques on the ferromagnets, and spin accumulations anywhere in the circuit can be computed this way.

As a specific application, consider the layered spinvalve structure and its effective circuit models sketched in Fig. 1. The reservoirs are described by electrochemical potentials $\mu_{c, L}$ and $\mu_{c, R}$. We can define four nodes on the normal side of each $F \mid N$ interface, with electrochemical potentials $\mu_{c, i}$ and spin accumulations $\boldsymbol{\mu}_{s, i}$. There are correspondingly three normal resistors in the problem, each described by a single real-valued conductance parameter $g$, and two magnetic resistors, corresponding to two ferromagnets, each described by two real-valued spin-dependent conductances $g^{\uparrow \uparrow}$ and $g^{\downarrow \downarrow}$ and four complex-valued spin-mixing conductances $g^{\uparrow \downarrow}$, $g^{\prime \uparrow \downarrow}$, and $t^{\uparrow \downarrow}, t^{\uparrow \uparrow \downarrow}$. In the absence of spin-orbit coupling, the charge and spin currents in each node depend on the electrochemical potentials and spin accumulations on each side of the respective ferromagnet according to Eqs. (25) and (26). Transport across normal resistors is described by simpler equations that could be easily obtained by setting $g^{\sigma \sigma^{\prime}}, g^{\prime \sigma \sigma^{\prime}}, t^{\sigma \sigma^{\prime}}, t^{\prime \sigma \sigma^{\prime}}$ to $g$ in Eqs. (25) and (26). By following the steps outlined above, these equations can be used to self-consistently determine $\mu_{c, i}$ and $\boldsymbol{\mu}_{s, i}$ and then the charge and spin currents into each node, as a function of the applied bias $\mu_{c, L}-\mu_{c, R}$. Obviously, the same procedure can be carried out for currentbiased instead of voltage-biased systems. When the conductance parameters are to be evaluated from first principles, the definitions (23) and (24) have to be corrected for kinetic effects when the interfaces are highly transmitting, as discussed in the next subsection. Placing of the nodes is to some extent arbitrary but different choices should not lead to contradictory results. For the system in Fig. 1, for example, it might be more convenient to replace nodes 2 and 3 with a single one somewhere in the middle of the normal spacer and eliminate nodes 1 and 4 altogether, while redefining the conductance matrices of the magnetic regions to include scattering on the normal-metal sides. In such a case, the entire structure would consist of two magnetic scatterers connecting each reservoir with the middle of the normal spacer. On the other hand, for sufficiently thick ferromagnets, we introduce four additional nodes on the ferromagnetic side of the interfaces and define pure $F \mid N$-interface resistances and ferromagnetic bulk resistances.

Circuit theory assumes momentum scattering in each node but not necessarily inelastic scattering. It is only required that the chemical-potential gradients and drops are small enough such that linear-response theory holds, and the energy dependence of the scattering matrix may be disregarded. Inelastic scattering in the nodes per definition does not affect the transport properties. It is, however, often stated that the scattering approach to transport is valid only when the orbital-dephasing length $\lambda_{\phi}$ is sufficiently longer than the dimensions of the scattering region. This is a relevant statement only when phase coherence is essential for the physics under consideration. In magnetoelectronic systems, we have to worry about orbital interference only at sharp interfaces. The spin-mixing conductances $g^{\uparrow \downarrow}, g^{\prime \uparrow \downarrow}$ and $t^{\uparrow \downarrow}, t^{\prime \uparrow \downarrow}$ in Eq. (26) govern transverse spin currents under the condition that $\lambda_{\phi} \gg \lambda_{\text {sc. }}$. Also, the description of conventional spindependent conductances by first-principles bandstructure calculations assumes wave-function coherence on atomistic length scales as well. These conditions are hardly restrictive and assumed to be valid up to high (room) temperature. When spin-flip scattering is strong in diffusive regions, the spin-diffusion equation should be solved there, for which Eqs. (25) and (26) provide the boundary conditions. Section IV.B demonstrates how this is carried out in practice. Although we have illustrated here circuit theory using simple linear structures, it can easily be applied to more general multiterminal devices, such as spin transistors.

\section{B. Interfacial and thin-film conductance matrices}

In the previous subsection, the electron states in the nodes were assumed to be occupied according to energy and spin, but without any regard to their momentum. Physically, this isotropy in momentum space implies that net currents in the nodes may be disregarded. This is allowed only when the incoming and outgoing currents do not significantly disturb the isotropic momentumspace distribution. When the contacts to the nodes are relatively small (point contacts) or highly resistive (tunnel junctions), this approximation holds. Highly conductive metallic multilayers that are the main subject of this review do not satisfy such a condition. The node interconnectors are then intermetallic interfaces or thin films. The isotropy of distribution functions in the nodes can be significantly distorted by the current induced at a given voltage bias. In that limit, simple Kirchhoff laws with Landauer-Büttiker conductances parameters, Eqs. (23) and (24), do not apply. This subsection summarizes how to rescue circuit theory by only modifying conductance parameters (Schep et al., 1997; Bauer et al., 2003b).

We first illustrate the issue for a nonmagnetic metallic pillar with a uniform cross section connecting two reservoirs. Transport through a ballistic pillar is governed by the Sharvin conductance $g^{\text {Sh }}$, i.e., the number of propagating transport channels. Let us introduce $M$ interfaces (e.g., grain boundaries) in series that scramble the transverse-momentum distribution of incident electrons without any significant backscattering. The total conductance must then still amount to $g^{\text {Sh }}$ since we excluded any reflection (Imry, 1997). If we naively carried out the circuit-theory protocol, we could assign a conductance $g_{i}=g^{\text {Sh }}$ to each interface and insert $M-1$ nodes between them. We obtain the total resistance $1 / g$ as the sum of the individual ones:

$$
\frac{1}{g}=\sum_{i=1}^{M} \frac{1}{g_{i}}=\frac{M}{g^{S h}},
$$

which is obviously wrong. This breakdown of the circuit theory can be "fixed" by renormalizing the individual 
interface resistance from scattering theory $1 / g_{i}$ by subtracting the Sharvin resistance $1 / g^{\mathrm{Sh}}$ :

$$
\frac{1}{\tilde{g}_{i}}=\frac{1}{g_{i}}-\frac{1}{g^{S h}} .
$$

At the same time, contact resistances between pillar and reservoirs are assigned half of their Sharvin resistance. We then arrive at the total resistance

$$
\frac{1}{g}=\frac{1}{2 g^{S h}}+\sum_{i=1}^{M} \frac{1}{\tilde{g}_{i}}+\frac{1}{2 g^{\mathrm{Sh}}}=\frac{1}{g^{\mathrm{Sh}}} .
$$

In this way, we forced a vanishing local voltage drop over each interface, corresponding to $\tilde{g}_{i}=\infty$, and a finite Sharvin resistance for the entire structure that is governed by the geometrical sample cross section. The total conductance is $g^{\text {Sh }}$, as it should be.

Schep et al. (1997) justified such renormalized resistances, deriving a more general result for interfaces between different materials:

$$
\frac{1}{\tilde{g}_{i}}=\frac{1}{g_{i}}-\frac{1}{2}\left(\frac{1}{g_{i, L}^{\mathrm{Sh}}}+\frac{1}{g_{i, R}^{\mathrm{Sh}}}\right),
$$

where $g_{i, L}^{\text {Sh }}$ and $g_{i, R}^{\text {Sh }}$ are Sharvin conductances on two sides of the interface, thus allowing a comparison between first-principles calculations of interface resistances with experimental results on diffuse multilayer systems that access $\tilde{g}_{i}$. Equation (32) requires global diffusivity that separately randomizes the momentum distributions for right- and left-moving electrons and destroys quantum interference between consecutive contacts.

The renormalization of resistances by subtracting Sharvin contributions is closely related to early controversies on resistance in mesoscopic systems. The bare Landauer-Büttiker conductance $g=\Sigma_{n n^{\prime}}\left|t_{n n^{\prime}}\right|^{2}$ is suitable for the description of the two-point measurement in which reservoirs are current as well as voltage sources (see, e.g., Imry, 1997). In an idealized four-point measurement in which the voltage drop is measured directly across the scatterer (e.g., an interface), the conductance should be renormalized since there is no geometric contribution. In a single-mode quantum wire with $g^{\mathrm{Sh}}=1$, the renormalized conductance reduces to the "old" Landauer formula $\tilde{g}=g /(1-g)$ (Landauer, 1970). When the scatterer is embedded in a diffuse environment and circuit theory applies, the situation is analogous to the four-point measurement and Eq. (32) should be used. In collinear magnetic structures, Eq. (32) should be applied for each spin channel separately (Schep et al., 1997).

The "kinetic" corrections for magnetic structures with noncollinear magnetizations require additional thought. As a starting point we consider a single $F \mid N$ interface as sketched in Fig. 3 with conductance matrices $g^{\sigma \sigma^{\prime}}$ and disregard spin-flip processes. In the presence of nonequilibrium currents, the effective electrochemical potentials and spin accumulations, Eqs. (15) and (16), on either side of the interface are different for left- and right-moving modes. The discussion of Sec. II.A must thus be modified to account for this asymmetry in the left- and right-moving distributions ("drift"). As in Schep et al. (1997), the central underlying assumption that allows an easy implementation of this program (Bauer et al., 2003a, 2003b) is a globally diffuse or chaotic system. In that case, the distribution of electrons incident on each interface from either side is isotropic in momentum space. Such a randomization is likely to be provided in realistic structures by interfacial disorder and bulk scattering. By extension of either the random matrix theory of Waintal et al. (2000) or circuit theory, the analysis (Bauer et al., 2003a, 2003b) then boils down to a set of simple rules. Equations (25) and (26) remain unchanged but the interfacial conductances are renormalized as

$$
\frac{1}{\tilde{g}^{\sigma \sigma^{\prime}}}=\frac{1}{g^{\sigma \sigma^{\prime}}}-\frac{1}{2}\left(\frac{1}{g_{N}^{\mathrm{Sh}}}+\frac{\delta_{\sigma \sigma^{\prime}}}{g_{F \sigma}^{\mathrm{Sh}}}\right),
$$

whereas the average electrochemical potentials and spin accumulations in the nodes have to be found selfconsistently as before. (We recall that the conductance parameter $t^{\prime \uparrow \downarrow}$ drops out of the discussion for a single $F \mid N$ interface.) Additionally, the leads to real reservoirs must be assigned half of the respective Sharvin resistances for each spin $\sigma$.

The renormalization (33) is significant for an understanding of the measured resistances of metallic multilayers in the current perpendicular to the interface planes configuration (Bass and Pratt, 1999), as well as other measurements, by ab initio calculations (Schep et al., 1997; Xia et al., 2001, 2002; Bauer et al., 2003b; Zwierzycki et al., 2005). In Table I we cite the theoretical results (Zwierzycki et al., 2005) for two representative $N \mid F$ material combinations: $\mathrm{Au} \mid \mathrm{Fe}(001)$ and $\mathrm{Cu} \mid \mathrm{Co}(111)$, the former routinely used by the Simon-Fraser group (e.g., Urban et al., 2001; Heinrich et al., 2002; Heinrich, Urban, et al., 2003) and the latter by the Cornell group (e.g., Myers et al., 1999; Katine et al., 2000). The large difference between the $\tilde{g}$ 's and $g$ 's is evident when using Eq. (33) as the bare interfacial conductances are comparable with the Sharvin conductances.

Equation (33) can be used only when the distribution function in the ferromagnetic layers is well defined. This is not the case anymore when the magnetic-film thickness is of the order or smaller than the transverse-spin coherence length (13). In the latter situation, calculations (Bauer et al., 2003a, 2003b) that lead to Eq. (33) have to be repeated for two normal-metal layers separated by a thin magnetic film as in Fig. 2. We find that the basic circuit-theory equations (25) and (26) hold for all film thicknesses after replacing the conductance parameters for the $N|F| N$ sandwiches with the renormalized ones, $\tilde{g}^{\sigma \sigma^{\prime}}$ and $\tilde{t}^{\sigma \sigma^{\prime}}$ : 


$$
\begin{aligned}
& \frac{1}{\tilde{g}^{\sigma \sigma}}=\frac{1}{g^{\sigma \sigma}}-\frac{1}{2}\left(\frac{1}{g_{N}^{\mathrm{Sh}}}+\frac{1}{g_{N}^{\prime \mathrm{Sh}}}\right), \\
& \frac{1}{\tilde{g}^{\uparrow \downarrow}}=\frac{1}{g^{\uparrow \downarrow}+t^{\uparrow \downarrow} t^{\prime \uparrow \downarrow} /\left(2 g_{N}^{\prime \mathrm{Sh}}-g^{\prime \uparrow \downarrow}\right)}-\frac{1}{2 g_{N}^{\mathrm{Sh}}}, \\
& \frac{1}{\tilde{t}^{\uparrow \downarrow}}=\frac{\left(2 g_{N}^{\mathrm{Sh}}-g^{\uparrow \downarrow}\right)\left(2 g_{N}^{\prime \mathrm{Sh}}-g^{\prime \uparrow \downarrow}\right) / t^{\uparrow \downarrow}-t^{\prime \uparrow \downarrow}}{4 g_{N}^{\mathrm{Sh}} g_{N}^{\prime \mathrm{Sh}}},
\end{aligned}
$$

and the same after interchanging $g \leftrightarrow g^{\prime}$ and $t \leftrightarrow t^{\prime}$.

In the presence of weak (compared to momentum scattering) spin-flip scattering during bulk diffusion, the same renormalizations, Eqs. (33) and (34), still hold for interfacial transport. In the opposite limit of high spinflip rates, the layers can act as ideal sinks for spin currents. In particular, in the regime of pure spin transport, such layers are fully equivalent to reservoirs, bearing all consequences and rules outlined in this subsection for the reservoirs. The regime of intermediate-strength spin dephasing cannot be characterized by such simple statements and is omitted from our discussion.

\section{Time-dependent theory}

In this review we are mostly interested in the dynamic phenomena stemming from a slow variation in the magnetization direction $\mathbf{m}$ of ferromagnets that are part of an Ohmic circuitry. We focus on the adiabatic response of the itinerant carriers to the time-dependent $\mathbf{m}$ that is driven by external magnetic fields or applied (spin) currents. It turns out that the magnetoelectronic (dc) circuit theory discussed above already contains all necessary parameters in the adiabatic regime. An adiabatic approximation is applicable when the frequency of the magnetization modulation is much smaller than the characteristic ferromagnetic exchange spin splitting, which is safely fulfilled for transition-metal-based structures. The total current is a sum of the currents induced by the bias applied via the reservoirs (including the spintransfer torques), viz., Eqs. (25) and (26), and the pumping component proportional to the rate of variation of the scattering potentials (Büttiker et al., 1994):

$$
\begin{aligned}
& I_{c}=I_{c}^{(0)}+I_{c}^{\mathrm{pump}}, \\
& \mathbf{I}_{s}=\mathbf{I}_{s}^{(0)}+\mathbf{I}_{s}^{\text {pump }} .
\end{aligned}
$$

We shall make it a convention to include in $I_{c}^{(0)}$ and $\mathbf{I}_{s}^{(0)}$ the currents driven by any charge and spin imbalance brought about by the pumping, as well as by the applied bias.

In Sec. III, we derive the currents pumped by a timedependent magnetization direction $\mathbf{m}$ into adjacent normal nodes:

$$
I_{c, R}^{\text {pump }}=0
$$

$$
\mathbf{I}_{s, R}^{\text {pump }}=\frac{\hbar}{4 \pi}\left(\mathcal{A}_{r}^{\uparrow \downarrow} \mathbf{m} \times \frac{d \mathbf{m}}{d t}+\mathcal{A}_{i}^{\uparrow \downarrow} \frac{d \mathbf{m}}{d t}\right),
$$

introducing a new complex-valued parameter

$$
\mathcal{A}^{\uparrow \downarrow}=\mathcal{A}_{r}^{\uparrow \downarrow}+i \mathcal{A}_{i}^{\uparrow \downarrow}=g^{\uparrow \downarrow}-t^{\prime \uparrow \downarrow}
$$

that determines the magnitude of the spin pumping as a function of device parameters (Tserkovnyak et al., 2002a). Magnetism (or another source of spin-dependent scattering) is essential for a nonvanishing pumping parameter $\mathcal{A}^{\uparrow \downarrow}$. The spin current into the left lead is similar to Eq. (38) and governed by $\mathcal{A}^{\prime \uparrow \downarrow}=g^{\prime \uparrow \downarrow}-t^{\uparrow \downarrow}$. When the ferromagnet is much thicker than the coherence length (13), the quantities $t^{\uparrow \downarrow}$ and $t^{\prime \uparrow \downarrow}$ vanish and the mixing conductances $g^{\uparrow \downarrow}$ and $g^{\prime \uparrow \downarrow}$ do not depend on the thickness of the ferromagnet; the spin-pumping currents originate from the interfaces. Spin pumping (38) thus does not depend on spin-flip processes in the ferromagnet when far from the $F \mid N$ interface on the scale of the coherence length (13) or, in other words, when the spinflip scattering rate is small compared to the exchange splitting. This is typically the case in real materials. The spin (de)coherence in the attached normal conductors is crucial, however, since it affects the self-consistent reaction torque exerted on a slowly precessing monodomain ferromagnet, as explained in Sec. IV.

It can be shown that the renormalizations (34) introduced in the dc circuit theory for layered structures must also be applied to the mixing conductances in $\mathcal{A}^{\uparrow \downarrow}$, Eq. (39), for multilayers with diffuse spin backscattering, but not for contacts to spin-sink reservoirs. Using Eqs. (34), for $\tilde{\mathcal{A}}^{\uparrow \downarrow}=\tilde{g}^{\uparrow \downarrow}-\tilde{t}^{\prime \uparrow \downarrow}$ we find

$$
\frac{\tilde{\mathcal{A}}^{\uparrow \downarrow}}{2 g_{N}^{\mathrm{Sh}}}=\frac{g^{\uparrow \downarrow}\left(2 g_{N}^{\prime \mathrm{Sh}}-g^{\prime \uparrow \downarrow}\right)-t^{\uparrow \downarrow}\left(2 g_{N}^{\prime S h}-t^{\prime \uparrow \downarrow}\right)}{\left(2 g_{N}^{\mathrm{Sh}}-g^{\uparrow \downarrow}\right)\left(2 g_{N}^{\prime \mathrm{Sh}}-g^{\prime \uparrow \downarrow}\right)-t^{\uparrow \downarrow} t^{\prime \uparrow \downarrow}},
$$

and the same after interchanging primed and unprimed quantities. For mirror-symmetric structures, Eq. (40) reduces to simply

$$
\frac{1}{\tilde{\mathcal{A}}^{\uparrow \downarrow}}=\frac{1}{\mathcal{A}^{\uparrow \downarrow}}-\frac{1}{2 g_{N}^{\mathrm{Sh}}} .
$$

The spin-pumping expression (38) sets the stage for several interesting developments reviewed in the following section. We therefore devote all of Sec. III to deriving Eq. (38) and discussing its physical content. See also Tserkovnyak et al. (2002a, 2000b, 2003a).

\section{SPIN EMISSION BY COHERENTLY PRECESSING FERROMAGNETS}

\section{A. Parametric spin pumping}

The adiabatic spin-pumping expression (38) can be derived using time-dependent scattering theory (Tserkovnyak et al., 2002a). Consider a $N|F| N$ junction as in Fig. 2, where the two normal nodes are now assumed to be large reservoirs in a common thermal equilibrium. Without a voltage bias, no spin or charge currents flow 
when the magnetization of the ferromagnet is static. When it is moving, however, the time dependence of the scattering matrix in spin space can induce nonequilibrium spin currents in the nonmagnetic leads. The current $\hat{I}(t)$ pumped by the precession of the magnetization into the right and left paramagnetic reservoirs can be calculated in the adiabatic approximation, since typical precession frequencies are several orders of magnitude smaller than the ferromagnetic exchange field that sets the relevant energy scale for spin-dependent transport. The adiabatic charge-current response in nonmagnetic systems has been derived by Büttiker et al. (1994). The generalization to the $2 \times 2$ spin- and charge-current matrix (17) by Tserkovnyak et al. (2002a, 2002b) is explained by the following.

The $2 \times 2$ current operator $\hat{I}_{l}$ for the $l$ th lead $(l$ $=L, R)$ is in general given by Eq. (18). When the scattering matrix $s_{n n^{\prime}, l l^{\prime}}^{\sigma \sigma^{\prime}}(t)$ of the ferromagnetic layer varies slowly compared to the relevant microscopic time scales of the system, an adiabatic approximation may be used, meaning that the energy of the scattered states is assumed to be weakly modulated with respect to the energy of the incoming states by the oscillating part of the scattering matrix. The state annihilated by $a_{n^{\prime}, l^{\prime}}^{\sigma^{\prime}}(\varepsilon, t)$ $=a_{n^{\prime}, l^{\prime}}^{\sigma^{\prime}}(\varepsilon) e^{-i \varepsilon t / \hbar}$ is partitioned into states in the $m$ th channel of lead $l^{\prime}$ with energies determined by the time dependence of $s_{n n^{\prime}, l l^{\prime}}^{\sigma \sigma^{\prime}}(\varepsilon, t) a_{n^{\prime}, l^{\prime}}^{\sigma^{\prime}}(\varepsilon, t)$; see Büttiker et al. (1994). The scattering amplitude at a given energy shift is determined by the Fourier transform of $s_{n n^{\prime}, l l^{\prime}}^{\sigma \sigma^{\prime}}(\varepsilon, t)$ in time space. The expectation value of the current operator $\hat{I}_{l}$ is evaluated similarly to the dc limit discussed in Sec. II.A. When the scattering matrix depends on the real-valued parameter $X(t)$, the Fourier transform of the current expectation value $\hat{I}_{l}(\omega)=\int d t e^{i \omega t} \hat{I}_{l}(t)$ can be written as

$$
\hat{I}_{l}(\omega)=\hat{g}_{X, l}(\omega) X(\omega)
$$

in terms of the frequency $\omega$ and $X$-dependent parameter $\hat{g}_{X, l}$ :

$$
\begin{aligned}
\hat{g}_{X, l}(\omega)= & \frac{e \omega}{4 \pi} \int d \varepsilon \frac{d f_{\mathrm{FD}}(\varepsilon)}{d \varepsilon} \sum_{n n^{\prime} l^{\prime}} \frac{\partial \hat{s}_{n n^{\prime}, l l^{\prime}}(\varepsilon)}{\partial X} \hat{s}_{n n^{\prime}, l l^{\prime}}^{\dagger}(\varepsilon) \\
& - \text { H.c. }
\end{aligned}
$$

Equation (42) is the first-order (in frequency) correction to the dc theory of Sec. II.A. At sufficiently low temperatures, one can approximate $-\partial_{\varepsilon} f_{\mathrm{FD}}(\varepsilon)$ by a $\delta$ function centered at the Fermi energy. The expectation value of the $2 \times 2$ particle-number operator $\hat{Q}_{l}(\omega)$ [defined by $\hat{I}_{l}(t)=d \hat{Q}_{l}(t) / d t$ in the time or by $\hat{I}_{l}(\omega)=-i \omega \hat{Q}_{l}(\omega)$ in the frequency domain] for the $l$ th reservoir is then given by

$$
i \hat{Q}_{l}(\omega)=\left(\frac{e}{4 \pi} \sum_{n n^{\prime} l^{\prime}} \frac{\partial \hat{s}_{n n^{\prime}, l l^{\prime}}}{\partial X} \hat{s}_{n n^{\prime}, l l^{\prime}}^{\dagger}-\text { H.c. }\right) X(\omega),
$$

where the scattering matrix is evaluated at the Fermi energy. Because the prefactor on the right-hand side of Eq. (44) does not depend on frequency $\omega$, the equation is also valid in the time domain. The change in particle number $\delta \hat{Q}_{l}(t)$ is thus proportional to the modulation $\delta X(t)$ of parameter $X$ and the $2 \times 2$ matrix current reads

$$
\hat{I}_{l}(t)=e \frac{d \hat{n}_{l}}{d X} \frac{d X(t)}{d t},
$$

introducing the matrix "emissivity" into lead $l$ :

$$
\frac{d \hat{n}_{l}}{d X}=\left(\frac{1}{4 \pi i} \sum_{n n^{\prime} l^{\prime}} \frac{\partial \hat{s}_{n n^{\prime}, l l^{\prime}}}{\partial X} \hat{s}_{n n^{\prime}, l l^{\prime}}^{\dagger}\right)+\text { H.c. }
$$

The right-hand side of Eq. (45) should in general be summed over all parameters, if any, that modulate the scattering matrix. When spin-flip scattering is disregarded, the scattering matrix $\hat{s}$ can be written in terms of spin-up and spin-down scattering coefficients $s^{\uparrow(\downarrow)}$ using projection matrices; see Eqs. (21) and (22). The spin current pumped by a magnetization precession is obtained by identifying $X(t)=\varphi(t)$, where $\varphi$ is the azimuthal angle of the magnetization direction in the plane perpendicular to the instantaneous rotation axis. For example, suppose the magnetization rotates around the $z$ axis: $\mathbf{m}$ $=(\cos \varphi, \sin \varphi, 0)$. Using Eq. (21) it is then easy to calculate the emissivity (46) for this process:

$$
\frac{d \hat{n}_{R}}{d \varphi}=\frac{1}{4 \pi}\left[\mathcal{A}_{i}^{\uparrow \downarrow}\left(\sigma_{x} \sin \varphi-\sigma_{y} \cos \varphi\right)-\mathcal{A}_{r}^{\uparrow \downarrow} \sigma_{z}\right] .
$$

Inserting this into Eq. (45) and comparing the result with the definition (17), one finally finds that the charge current vanishes, $I_{c, R}^{\mathrm{pump}}=0$, and the spin current

$$
\mathbf{I}_{s, R}^{\text {pump }}=\left(-\mathcal{A}_{i}^{\uparrow \downarrow} \sin \varphi, \mathcal{A}_{i}^{\uparrow \downarrow} \cos \varphi, \mathcal{A}_{r}^{\uparrow \downarrow}\right) \frac{\hbar}{4 \pi} \frac{d \varphi}{d t}
$$

can be rewritten as Eq. (38). Since the spin current transforms as a vector in spin space, Eq. (38) is also valid in the case of a general (slow) motion of the magnetization direction.

Even though the mathematics of the scattering approach to adiabatic spin pumping is entirely analogous to the charge-pumping theory developed by Büttiker et al. (1994) and Brouwer (1998), there is an important difference in the physics. In the case of a nonmagnetic scattering region, the average pumped charge current has the same direction in the two leads because charge cannot be accumulated or depleted for long; the net charge entering the scattering region through one lead must leave it via the other within a period of the external-gate modulation. On the other hand, the total conductionelectron-spin angular momentum does not have to be conserved since the magnetization can act as a sink or a source. The preceding analysis shows that a precessing ferromagnet polarizes adjacent nonmagnetic conductors. 
The phenomenon can be called as well a spin well or spin fountain rather than a spin pump. An excited ferromagnet emits spins into all adjacent conductors, spending its "own" angular momentum rather than pumping spins from one lead into the other. If the lost angular momentum is not replenished by an external magnetic field, the precession invariably will die out.

Per revolution, the precession pumps an angular momentum into an adjacent normal-metal layer, which is proportional to $\mathcal{A}_{r}^{\uparrow \downarrow}$, in the direction of the precession axis. At first sight, it may be surprising that a net dc pumping can be achieved by a single parameter varying in time, whereas it has been shown (Brouwer, 1998) that at least two periodic parameters are required for that. However, there are actually two periodic parameters (out of phase by $\pi / 2$ ) hidden behind $\varphi(t)$, viz., the projections of the unit vector defined by $\varphi$ in the plane perpendicular to the axis of precession.

\section{B. Rotating-frame analysis}

It turns out that Eqs. (38) and (39) for the nonequilibrium spin currents pumped out of a precessing ferromagnet can be understood as an equilibrium property by a transformation to a rotating frame. We prove this for a ferromagnetic film sandwiched between two normalmetal nodes at a common thermal equilibrium, $\mu_{c, L}$ $=\mu_{c, R}$, as shown in Fig. 2. Let

$$
H(t)=H_{0}+H^{\prime}(t)
$$

be the Hamiltonian experienced by conduction electrons, where $H_{0}$ is the sum of the kinetic and potential energies and $H^{\prime}(t)$ is a time-dependent exchange Hamiltonian in the ferromagnet:

$$
H^{\prime}(t)=\int d \mathbf{r} V_{x}(\mathbf{r}) \sum_{\sigma \sigma^{\prime}} \Psi_{\sigma}^{\dagger}(\mathbf{r})\left[\sigma^{\sigma \sigma^{\prime}} \cdot \mathbf{m}(\mathbf{r}, t)\right] \Psi_{\sigma^{\prime}}(\mathbf{r}),
$$

where $V_{x}$ is the local exchange interaction along the magnetization direction $\mathbf{m}(\mathbf{r}, t)=\mathbf{m}(t)$ that is assumed to be uniform in the ferromagnet. $\Psi_{\sigma}$ is the spin- $\sigma$ electron field operator.

At time $t<0$, the entire system is in a common thermal-equilibrium state corresponding to a timeindependent magnetization direction $\mathbf{m}(0)$. At $t=0, \mathbf{m}(t)$ starts to rotate with frequency $\omega$ about an axis denoted $\hat{\mathbf{z}}$ that is perpendicular to $\mathbf{m}, \dot{\mathbf{m}}=\omega \hat{\mathbf{z}} \times \mathbf{m}$. The timedependent magnetization drives the electron system out of equilibrium.

Let us introduce a (many-body) state $\Phi^{\prime}(t)$ of the electrons in the rotating frame of reference for the spin variables, which is related to the solution of the Schrödinger equation $i \hbar \dot{\Phi}(t)=H(t) \Phi(t)$ by the unitary transformation $\Phi(t)=U(t) \Phi^{\prime}(t)$. Here, $U=\exp \left(-i S_{z} \omega t / \hbar\right)$ is a spinrotation operator in terms of the total-spin $z$-axis projection $S_{z}$. Since $H^{\prime}(t)=U(t) H^{\prime}(0) U^{\dagger}(t), \Phi^{\prime}(t)$ satisfies a Schrödinger equation (Slichter, 1990):

$$
i \hbar \frac{d \Phi^{\prime}(t)}{d t}=\left[H(0)-S_{z} \omega\right] \Phi^{\prime}(t)
$$

provided $\left[H_{0}, S_{z}\right]=0$, i.e., spin-orbit coupling or other spin-flip processes are disregarded. This is the equation of motion for electrons subjected to a static magnetic field $\omega / \gamma$ in the $z$ direction, in addition to a static exchange interaction (50) along $\mathbf{m}(0)$ in the ferromagnet. A steady-state solution for the system in the rotating frame is thus characterized by spin-polarized normalmetal nodes with $\left\langle s_{z}\right\rangle=\chi \omega / \gamma$ and $\left\langle s_{x}\right\rangle=\left\langle s_{y}\right\rangle=0$, where $\chi$ is their static (isotropic) spin susceptibility.

In the laboratory frame, the spin imbalance must be identical to the polarization in the rotating frame along the $z$ axis. Since there is no magnetic field in the laboratory frame, this spin imbalance can only be accounted for by a nonequilibrium spin accumulation, i.e., a difference between the chemical potentials for spins parallel and antiparallel to $\hat{\mathbf{z}}$ of magnitude

$$
\mu_{s}=\hbar \omega \text {. }
$$

According to the magnetoelectronic dc circuit theory, the spin accumulation in turn drives a spin current

$$
\mathbf{I}_{s, R}^{\text {back }}=\frac{1}{4 \pi}\left(\mathcal{A}_{r}^{\uparrow \downarrow} \boldsymbol{\mu}_{s}+\mathcal{A}_{i}^{\uparrow \downarrow} \boldsymbol{\mu}_{s} \times \mathbf{m}\right)
$$

from a normal-metal node back into the ferromagnet, where the coefficients $\mathcal{A}^{\uparrow \downarrow}$ have been defined in Eq. (39). Here, we have used Eq. (26) with $\mu_{c, L}-\mu_{c, R}=0$ and $\boldsymbol{\mu}_{s, L}=\boldsymbol{\mu}_{s, R}=\boldsymbol{\mu}_{s}$. According to Eq. (52), $\boldsymbol{\mu}_{s}=\hbar \omega \hat{\mathbf{z}}=\hbar \mathbf{m} \times \dot{\mathbf{m}}$. Since in the steady state the backflow spin current (53) must cancel the spin current pumped by the ferromagnet into the normal metal, the above arguments thus provide an alternative derivation of the spin pumping (38). For finite-size nodes and slow rotation, equilibrium may be established in the rotating frame in a time that is short compared to the period of rotation. In the adiabatic limit, the equality of the spin currents into and out of the ferromagnet thus holds instantaneously, and not just over the average over a period. The assumption of a fixed rotation axis is also not essential, as long as this axis moves slowly in time. In the limit of vanishing exchange splitting, $\mathcal{A}^{\uparrow \downarrow}=0$ and the spin current (38) vanishes, as it should.

It is instructive to scrutinize the energy and spin angular-momentum conservation laws of the combined $F \mid N$ system in the laboratory frame of reference (Tserkovnyak et al., 2002b). Suppose the rotation axis $z$ is oriented along the static effective magnetic field $\mathbf{H}_{\text {eff }}$, and let us focus on the nonequilibrium dynamics in the normal metal node; see Fig. 4. After starting the magnetization rotation at $t=0$, net spin currents flow into the normal mode in a finite time interval before reaching the steady state. We wish to account for the energy and angular-momentum transfer between the ferromagnet and normal metal in this transient regime. If the normal node is sufficiently small, the steady state is reached after a vanishingly small transfer of spins, with only little effect on the ferromagnet. $N_{s}$ spins oriented along the $z$ 


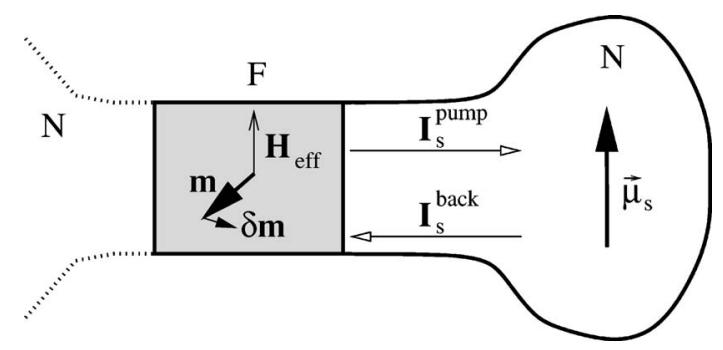

FIG. 4. The ferromagnetic magnetization $\mathbf{m}$ rotates around the effective field $\mathbf{H}_{\text {eff. }}$ In the steady state, the spin pumping $\mathbf{I}_{s}^{\text {pump }}$ cancels the spin backflow $\mathbf{I}_{s}^{\text {back }}$ that accompanies the nonequilibrium spin accumulation $\boldsymbol{\mu}_{s}=\hbar \gamma \mathbf{H}_{\mathrm{eff}}$.

axis transferred into the normal metal correspond to an excess energy $\Delta E_{N}=N_{s} \mu_{s} / 2$ and angular momentum $\Delta L_{N}=N_{s} \hbar / 2$. Conservation laws dictate $\Delta E_{F}=-\Delta E_{N}$ and $\Delta L_{F}=-\Delta L_{N}$ for the ferromagnet. Using Eq. (6), the magnetic energy $\Delta E_{F}=\gamma \Delta L_{F} H_{\text {eff }}$ and we find $N_{s} \mu_{s} / 2$ $=\gamma N_{s}(\hbar / 2) H_{\text {eff }}$. It follows that $\mu_{s}=\hbar \gamma H_{\text {eff }}=\hbar \omega$, where $\omega$ $=\gamma H_{\text {eff }}$ is the Larmor precession frequency in the effective field. Using energy and momentum conservation, we have thus derived a nonequilibrium spin accumulation that agrees with the result of the rotating-frame analysis.

\section{FMR-operated spin battery}

In the preceding subsection, we showed that precessing ferromagnets inject a spin current into adjacent conductors via Ohmic contacts. In this subsection, we discuss how this opens the way to creating a pure spin source ("spin battery") by ferromagnetic resonance (FMR) and estimate the spin current and spin bias for different material combinations (Brataas et al., 2002). The spin source can be realized as a ferromagnetic layer at resonance with a rf field. Pure spin-current injection into low-density conductors should allow experimental studies of spintronic phenomena in mesoscopic, ballistic, and nanoscale systems. The combination of a ferromagnet at the FMR in Ohmic contact with a conductor can be interpreted as a spin battery, with analogies and differences with charge batteries. For example, chargecurrent conservation dictates that a charge battery has two poles, plus and minus. A spin battery requires only one pole, since the spin current does not need to be conserved. Furthermore, the polarity is not a binary, but a three-dimensional vector.

Devices made from metallic layered systems displaying the giant (Baibich et al., 1988) and tunnel (Miyazaki and Tezuka, 1995; Moodera et al., 1995) magnetoresistance have been proven useful for read-head sensors and magnetic random-access memories. Integration of such devices with semiconductor electronics is desirable but difficult because a large conductivity mismatch between magnetic and normal materials is detrimental to spin injection (Schmidt et al., 2000). Spin injection into bulk semiconductors has so far been reported only in optical pump-and-probe experiments (Kikkawa and Aw- schalom, 1999) and with high-resistance ferromagnetic injectors (Fiederling et al., 1999; Ohno et al., 1999) or Schottky or tunnel barriers (Monsma et al., 1998; Zhu et al., 2001). In these cases, however, the injected spinpolarized carriers are hot and currents are small. Desirable are semiconductor devices with an efficient allelectrical cold-electron spin injection and detection via Ohmic contacts at the Fermi energy, recently realized by Jedema et al. (2001) for metallic devices. The spin battery discussed here is an alternative conceptual approach to accomplishing such electrical spin injection.

In the absence of spin-orbit scattering, the spinbattery idea can be understood in terms of the rotatingframe analysis of the previous subsection. A magnetization undergoing a circular precession around the $z$ axis with frequency $\omega$ induces a spin-imbalance density $\mathbf{s}$ $=\chi \omega \hat{\mathbf{z}} / \gamma$ in the normal metal or semiconductor adjacent to the ferromagnet. The spin susceptibility $\chi$ includes possible effects of electron-electron interactions in the normal metal-semiconductor. The chemical-potential difference (52) between spin-up and spin-down electrons along the $z$ axis, on the other hand, is universal for the FMR-operated spin battery with vanishing spin-orbit coupling. Spin-orbit scattering limits the efficiency of the spin battery in real structures, as discussed in the following.

The important parameters of a charge battery are the maximum voltage in the absence of a load, as well as the maximum charge current that can be drawn from it. In the following we discuss estimates for the analogous characteristics of the spin battery, closely following Brataas et al. (2002). To this end, one should solve the dynamic problem of spin pumping (38) at the $F \mid N$ contact as a boundary condition for the spin-diffusion equations in the normal conductor. When the ferromagnet is thicker than the ferromagnetic coherence length (13) (a few $\AA$ in transition metals such as $\mathrm{Co}, \mathrm{Ni}$, or $\mathrm{Fe}$ ), the spin current (38) emitted into the normal conductor is determined by the mixing conductance $g^{\uparrow \downarrow}$ since $t^{\uparrow \downarrow}$ vanishes. With some exceptions such as ferromagnetic insulators (Huertas-Hernando et al., 2002), the imaginary part of the mixing conductance is small (Xia et al., 2002), see Table I, and will be disregarded in the following. The spin current emitted into the normal metal is then

$$
\mathbf{I}_{s}^{\text {pump }}=\frac{\hbar}{4 \pi} g_{r}^{\uparrow \downarrow} \mathbf{m} \times \frac{d \mathbf{m}}{d t} .
$$

When the spin current (54) flows freely into the normal metal, the corresponding loss of angular momentum increases the (Gilbert) damping of the magnetization dynamics, as discussed in detail in the next section. Equation (54) is the maximum spin current that can be drawn from the spin battery. The rotating-frame analysis indicates that a nonequilibrium spin accumulation builds up in the normal conductor when the spin-flip relaxation rate is smaller than the spin-injection rate. This in turn induces a "backflow" spin current $\mathbf{I}_{s}^{(0)}$ that opposes the total spin current $\mathbf{I}_{s}=\mathbf{I}_{s}^{\text {pump }}+\mathbf{I}_{s}^{(0)}$. The component of the backflow spin current $\mathbf{I}_{s}^{(0)}$ parallel to the instantaneous 
magnetization direction $\mathbf{m}$ is canceled by an opposite flow from the ferromagnet when the FMR frequency and spin-flip scattering rate in the ferromagnet are much smaller than the characteristic spin-injection rate into the ferromagnet. The surviving component of $\mathbf{I}_{s}^{(0)}$, Eq. (26), is perpendicular to $\mathbf{m}$ :

$$
\mathbf{I}_{s}^{(0)}=-\frac{g_{r}^{\uparrow \downarrow}}{4 \pi} \mathbf{m} \times \boldsymbol{\mu}_{s} \times \mathbf{m} .
$$

We note that the mixing conductance in Eqs. (54) and (55) ought to be renormalized in layered structures, as discussed in Sec. II.B.

The relation between the spin accumulation $\boldsymbol{\mu}_{s}$ and the total spin current $\mathbf{I}_{s}$ in a normal diffuse conductor is governed by the spin-diffusion equation; see Sec. IV.B. Let us consider a $F \mid N$ bilayer structure with cross section $S$ and thickness $L$, diffusion coefficient $D$, and characteristic spin-flip time $\tau_{\text {sf }}$ for the $N$ layer. The solution for the spin accumulation is simple when $\sqrt{D / \omega} \ll L$ $\ll \sqrt{D \tau_{\mathrm{sf}}}=\lambda_{\text {sd }}$, requiring $\omega \gg \tau_{\text {sf }}^{-1}$ (Brataas et al., 2002). The spin accumulation in the normal layer is then nearly uniform and time independent for a steady ferromagnetic precession cone with angle $\theta$ :

$$
\boldsymbol{\mu}_{s}=\hbar \omega \frac{\sin ^{2} \theta}{\sin ^{2} \theta+\eta} \hat{\mathbf{z}}
$$

$\eta=\tau_{i} / \tau_{\mathrm{sf}}$ is a reduction factor expressed in terms of the injection rate $\tau_{i}^{-1}$. When the conductance of a normallayer slab of thickness $\sqrt{D / \omega}$ is much larger than the contact conductance $g_{r}^{\uparrow \downarrow}, \tau_{i}^{-1}=g_{r}^{\uparrow \downarrow} / h \mathcal{N} S L$, where $\mathcal{N}$ is the normal-metal one-spin density of states per unit volume. In the opposite limit, $\tau_{i}^{-1}=\sqrt{D \omega} / L$. The former regime is relevant for an opaque interface, such as a Schottky or tunnel barrier. In the latter regime, $\eta \ll 1$ since we have

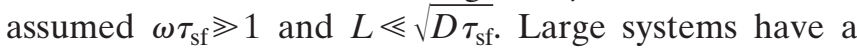
smaller injection rate since more states have to be filled. When $\tau_{\mathrm{sf}} \rightarrow \infty, \eta=0$ and Eq. (56) reduces to the result of the rotating-frame analysis (52). Schmidt et al. (2000) realized that efficient spin injection into semiconductors by Ohmic contacts is difficult with transition-metal ferromagnets since virtually all of the applied potential drops over the nonmagnetic part and is unavailable for spin injection. The present mechanism of spin injection is current rather than bias driven and thus does not suffer from this conductivity-mismatch problem.

When the spin-relaxation rate is smaller than the spininjection rate and the precession-cone angle is sufficiently large, $\sin ^{2} \theta>\eta$, the spin bias (56) saturates at its maximum value (52), which does not depend on the material parameters. At resonance, $\sin \theta=h_{\mathrm{rf}} / \alpha H_{\text {eff }}$ (assuming for simplicity a constant effective field $\left.\mathbf{H}_{\mathrm{eff}}\right)$. For a dc field of $H_{\mathrm{eff}}=1 \mathrm{~T}$, rf field $h_{\mathrm{rf}}=1 \mathrm{mT}$, and damping $\alpha$ $=0.01, \sin ^{2} \theta=0.01$. In order to realize a battery with maximum spin bias (52), a suppression factor $\eta \leq 0.01$ is thus required. Epitaxially grown clean samples with low spin-flip rates are needed for spin batteries operating at small precession angles. The precession-cone angle $\theta$ in FMR experiments is typically small, but large angles could in principle be achieved by sufficiently intense rf fields and a soft ferromagnet such as Permalloy or, for transient dynamics, by transverse magnetic-field pulses.

The dc component of the maximum spin-current bias (54) is suppressed by $\sin ^{2} \theta$ upon averaging over one precession period. For operation as a spin-current source, large-angle precession is required as well. The current can be increased by a larger $F \mid N$ interface area.

A potential practical problem of large-angle dynamics is the FMR energy dissipation. For a total spin $S_{F}$ $=M_{s} V / \gamma$ of a ferromagnet of volume $V$, the dissipation power $P=\alpha S_{F} \omega^{2} \sin ^{2} \theta$ has to be efficiently drained in order to avoid excessive heating. On the other hand, possible undesirable spin precession and energy generation in the nonmagnetic parts of the system is of no concern for material combinations with different $g$ factors, as, e.g., Fe $(g=2.1)$ and GaAs $(g=-0.4)$, or when the magnetic anisotropy shifts the resonance frequency with respect to electrons in the normal metal.

Purified elemental metals such as $\mathrm{Al}$ and $\mathrm{Cu}$ are suitable materials since their spin-diffusion lengths are very long. In $\mathrm{Cu}, \lambda_{\mathrm{sd}} \sim 1 \mu \mathrm{m}$ at low temperatures that is reduced by a factor of 3 at room temperature (Jedema $e t$ al., 2001), and similar numbers hold for Al (Jedema et al., 2002). Indirect proof of a spin accumulation pumped into $\mathrm{Cu}$ is provided by the FMR measurements of Permalloy $|\mathrm{Cu}| \mathrm{Pt}$ structures (Mizukami et al., 2002a, $2002 b)$ discussed in Sec. IV.B. Semiconductors have the advantage of a larger ratio of spin bias to Fermi energy. In lightly $n$-doped GaAs, the spin-flip relaxation time can be very long: $\tau_{s}=10^{-7} \mathrm{~s}$ at $n=10^{16} \mathrm{~cm}^{-3}$ carrier density (Kikkawa and Awschalom, 1998). This favorable number is offset by the difficulty of forming Ohmic contacts to GaAs, however. Large Schottky barriers exponentially suppress the interface mixing conductance. InAs has the advantage of a natural charge accumulation layer at the surface that avoids Schottky barriers when covered by high-density metals. However, the spin-orbit interaction in a narrow-gap semiconductor such as InAs is substantial, which reduces $\tau_{\mathrm{sf}}$. In asymmetric-confinement structures, the spin-flip relaxation rate is governed by the Rashba-type spin-orbit interaction that vanishes in symmetric quantum wells (Engels et al., 1997; Nitta et al., 1997). The remaining D'yakonov-Perel scattering rate (D'yakonov and Perel, 1971) is reduced in narrow quasi-one-dimensional channels of width $w$, due to waveguide diffusion modes, by a factor of $\left(L_{s} / w\right)^{2}$, where $L_{s}$ is the spin-precession length (Mal'shukov and Chao, 2000). This makes InAs and its alloys potentially interesting materials for a spin battery as well. In pure $\mathrm{Si}$, the spin-flip relaxation time should be very long since spin-orbit interaction is weak. Furthermore, the possibility of heavy doping allows control of Schottky barriers. Si therefore also appears to be a good candidate for spin injection into semiconductors.

The FMR-generated spin bias can be detected noninvasively via a ferromagnet that is connected to the normal metal by a tunnel junction. A voltage difference $p \mu_{s}$ should be measurable between parallel and antiparallel 
configurations of the analyzing magnetization with respect to the spin accumulation in the normal metal. Here $p=\left(G_{\uparrow}-G_{\downarrow}\right) /\left(G_{\uparrow}+G_{\downarrow}\right)$ is the relative polarization of the spin-dependent tunnel conductance $G^{\sigma}$ of the contact. For a fixed analyzing magnetization, the same voltage difference can be generated by reversing the static magnetic field. The spin current, on the other hand, can be measured via the drop of spin bias over a known resistive element with weak spin-flip scattering.

Spin pumping into the normal metal can also have consequences for nuclei via the hyperfine interaction between electrons and nuclear spins (Kawakami et al., 2001). An initially unpolarized collection of nuclear spins can be oriented by a spin-polarized electron current, which transfers part of its angular momentum by spin-flop scattering due to the hyperfine interaction. A ferromagnetically ordered nuclear-spin system, in turn, is felt by the electrons as the Overhauser magnetic field which induces an equilibrium spin density $\mathbf{s}_{0}$ in the normal metal (Overhauser, 1953). The spin-flop scattering can be included in electron-spin dynamics by adding an additional term to the total spin current, $\mathbf{I}_{s}=\mathbf{I}_{s}^{\text {pump }}+\mathbf{I}_{s}^{(0)}$ $+\mathbf{I}_{s}^{\text {nuc }}$, to account for the exchange of angular momentum between electrons and nuclei (Brataas et al., 2002). The nuclear spin polarization increases with spin accumulation and decreasing temperature. In bulk GaAs, the nuclear magnetic field is $H_{n}=5.3 \mathrm{~T}$ when the nuclei are fully spin polarized (Paget et al., 1977), which should occur at thermal energies sufficiently smaller than the FMR frequency, as determined from the rotating-frame analysis.

In conclusion, the spin battery is a source of spin, just as a conventional battery is a source of charge. Estimates of its performance for bilayers of metallic ferromagnets with either normal metals or doped semiconductors suggest that it is a feasible concept.

\section{GILBERT-DAMPING ENHANCEMENT}

\section{A. Ideal spin sinks}

In Sec. III we have shown that a moving magnetization emits spins into adjacent nonmagnetic conductors. This effect is necessarily associated with an energy loss for the ferromagnet, as explained in Sec. III.B. We now discuss spin pumping as a source of viscous damping of the magnetization dynamics in thin films or small particles. Under quite general conditions, this damping is consistent with the Gilbert phenomenology (8).

Consider a ferromagnet sandwiched between two normal metals labeled by $l=L, R$. By conservation of angular momentum, spins ejected out of the ferromagnet exert a transverse reaction torque (12). The total spin current

$$
\mathbf{I}_{s}=\sum_{l}\left(\mathbf{I}_{s, l}^{(0)}+\mathbf{I}_{s, l}^{\mathrm{pump}}\right)
$$

that determines the spin torque, i.e., the additional term (14) in the Landau-Lifshitz-Gilbert equation, contains bias- and spin-pumping-induced contributions. Suppose initially that normal metals act as ideal reservoirs in a common thermal equilibrium that are perfect spin sinks for pumped spin currents, so that $\mathbf{I}_{s, l}^{(0)}=\mathbf{0}$. This model is valid when the spin current $\mathbf{I}_{s, l}^{\text {pump }}$ is completely drained by massive and highly conductive reservoirs or by a material with effective spin-flip processes that prevent any spin-accumulation buildup and backscattering into the ferromagnet. Using Eq. (38),

$$
\begin{aligned}
\mathbf{I}_{s}= & \sum_{l} \mathbf{I}_{s, l}^{\text {pump }}=\frac{\hbar}{4 \pi}\left[\left(\mathcal{A}_{r}^{\uparrow \downarrow}+\mathcal{A}_{r}^{\prime \uparrow \downarrow}\right) \mathbf{m} \times \frac{d \mathbf{m}}{d t}\right. \\
& \left.+\left(\mathcal{A}_{i}^{\uparrow \downarrow}+\mathcal{A}_{i}^{\prime \uparrow \downarrow}\right) \frac{d \mathbf{m}}{d t}\right] .
\end{aligned}
$$

Since this spin current is polarized perpendicularly to $\mathbf{m}$, the torque (12) becomes $\boldsymbol{\tau}=-\mathbf{m} \times \mathbf{I}_{s} \times \mathbf{m}=-\mathbf{I}_{s}$. Substituting this into Eq. (14), we recover the Landau-LifshitzGilbert equation (8) with a new effective damping constant $\alpha_{\text {eff }}$ and gyromagnetic ratio $\gamma_{\text {eff }}$ defined by

$$
\begin{aligned}
& \frac{\gamma}{\gamma_{\text {eff }}}=1-\frac{\hbar \gamma}{4 \pi M_{s} V}\left(\mathcal{A}_{i}^{\uparrow \downarrow}+\mathcal{A}_{i}^{\prime \uparrow \downarrow}\right), \\
& \alpha_{\text {eff }} \frac{\gamma}{\gamma_{\text {eff }}}=\alpha+\frac{\hbar \gamma}{4 \pi M_{s} V}\left(\mathcal{A}_{r}^{\uparrow \downarrow}+\mathcal{A}_{r}^{\prime \uparrow \downarrow}\right) .
\end{aligned}
$$

Spin pumping thus affects FMR experiments as a shift of the resonance magnetic field via $\mathcal{A}_{i}^{\uparrow \downarrow}+\mathcal{A}_{i}^{\uparrow \uparrow \downarrow}$, whereas $\mathcal{A}_{r}^{\uparrow \downarrow}+\mathcal{A}_{r}^{\prime \uparrow \downarrow}$ increases the relative resonance linewidth. $\mathcal{A}_{r}^{\uparrow \downarrow}$ defined by Eq. (39) is positive since, by unitarity of the scattering matrix, it can be rewritten as

$$
\mathcal{A}_{r}^{\uparrow \downarrow}=\frac{1}{2} \sum_{n n^{\prime}}\left(\left|r_{n n^{\prime}}^{\uparrow}-r_{n n^{\prime}}^{\downarrow}\right|^{2}+\left|t_{n n^{\prime}}^{\uparrow}-t_{n n^{\prime}}^{\prime}\right|^{2}\right) \text {. }
$$

Spin pumping by a moving magnetization therefore cannot reverse the sign of the effective damping parameter (without reversing the sign of the gyromagnetic ratio), as is required by the Landau-Lifshitz-Gilbert phenomenology; see Sec. I.C.

Enhanced Gilbert damping leads to a broader resonance absorption peak (11) of a rf magnetic field. By the fluctuation-dissipation theorem, this should be manifest in increased fluctuations of the magnetization in thermodynamic equilibrium. This additional magnetization noise can arise from the torques exerted by JohnsonNyquist spin-current fluctuations that are exchanged between the ferromagnet and reservoirs or other spin sinks. Indeed Foros, Brataas, et al. (2005) have proven explicitly that magnetic noise caused by spin-current fluctuations is consistent with the dissipation power predicted by spin-pumping theory for a monodomain ferromagnet in contact with ideal spin sinks.

Mizukami et al. (2001a, 2001b) measured roomtemperature FMR linewidths of sputtered $N \mid$ Permalloy $\left(\mathrm{Ni}_{80} \mathrm{Fe}_{20}, \mathrm{Py}\right) \mid N$ sandwiches and discovered systematic trends in the damping parameter as a function of Py-film thickness $d$ for different normal metals $N$. Their data for the Gilbert parameter $G$, Eq. (9), are shown by symbols in Fig. 5. In the following, we compare these measure- 


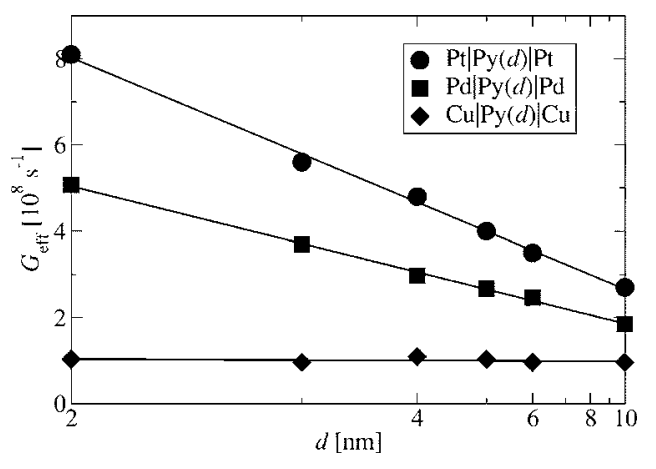

FIG. 5. Symbols represent the data points derived from FMR experiments on $N|\mathrm{Py}| N$ sandwiches with $N=\mathrm{Pt}, \mathrm{Pd}$, or $\mathrm{Cu}(\mathrm{Mi}-$ zukami et al., 2001a, 2001b). The lines show fits by $G+a / d$. $G=0.97 \times 10^{8}, 1.06 \times 10^{8}$, and $1.31 \times 10^{8} \mathrm{~s}^{-1}$, where, following Eq. (63), $a$ corresponds to $g_{r}^{\uparrow \downarrow} / S=0.2,15.3$, and $25.8 \mathrm{~nm}^{-2}$ for $\mathrm{Cu}, \mathrm{Pd}$, and $\mathrm{Pt}$, respectively, taking a $g$ factor of 2.1 for Py films (Mizukami et al., 2001a, 2001b). The horizontal axis uses a reciprocal scale.

ments with theoretical predictions based on Eq. (60). For the Py films, $d \geqslant 2 \mathrm{~nm} \gg \lambda_{\text {sc }}$, so that $t^{\uparrow \downarrow}, t^{\prime \uparrow \downarrow} \approx 0$ and $\mathcal{A}^{\uparrow \downarrow}$, Eq. (39), is simply the mixing conductance $g^{\uparrow \downarrow}$ of the Py $\mid N$ interface. $g_{i}^{\uparrow \downarrow} \ll g_{r}^{\uparrow \downarrow}$ for the interfaces listed in Table I, so that

$$
g^{\uparrow \downarrow} \approx g_{r}^{\uparrow \downarrow} .
$$

Equation (59) then reduces to $\gamma_{\mathrm{eff}} \approx \gamma$ and Eq. (60) becomes

$$
G_{\mathrm{eff}} \approx G+\frac{\hbar \gamma^{2} g_{r}^{\uparrow \downarrow}}{2 \pi V}
$$

Moreover, for the parameters in Table I,

$$
g_{r}^{\uparrow \downarrow} \approx g_{N}^{\mathrm{Sh}} .
$$

As explained by Zwierzycki et al. (2005), Eqs. (62) and (64) are good approximations for intermetallic interfaces because of the large phase differences between spin-up and spin-down reflection coefficients (see, however, Sec. VII.A.2 for ultrathin magnetic films, $d \leq \lambda_{\text {sc }}$ ). We shall assume the validity of these approximations in much of this review. Sharvin conductances for different normal metals (Zwierzycki et al., 2005) are listed in Table II. The data in Fig. 5 are used to extract $g_{r}^{\uparrow \downarrow}$ according to Eq. (63). A possible $d$ dependence of the bulk $G$ is disre-

TABLE II. Sharvin conductances (in units of quantum channels per $\mathrm{nm}^{2}$ ) for bulk fcc $\mathrm{Cu}, \mathrm{Pd}, \mathrm{Pt}$, and bcc Ta, density of states $\mathcal{N}$ at the Fermi level (in units of states per Ry, atom, spin), and Stoner enhancement factor. From Zwierzycki et al., 2005. Typical values of the exchange-correlation integral $I_{\mathrm{xc}}$ were taken from Gunnarsson, 1976 and Janak, 1977.

\begin{tabular}{ccccc}
\hline \hline & $\mathrm{Cu}$ & $\mathrm{Ta}$ & $\mathrm{Pd}$ & $\mathrm{Pt}$ \\
\hline$g^{\mathrm{Sh}}$ & 15.0 & 25.0 & 16.0 & 17.6 \\
$\mathcal{N}$ & 2 & 10 & 15 & 12 \\
$\left(1-\mathcal{N} I_{\mathrm{xc}}\right)^{-1}$ & 1.1 & 1.9 & 4.4 & 2.2 \\
\hline \hline
\end{tabular}

garded in our analysis. The $G$ 's in Fig. 5 are close to the previously measured bulk Gilbert damping constants of Permalloy (Patton et al., 1975; Bastian and Biller, 1976). We see that the extracted mixing conductance for the $\mathrm{Pd} \mid \mathrm{Py}$ interface is similar to that expected from the Sharvin conductance of $\mathrm{Pd}$, while that of $\mathrm{Pt} / \mathrm{Py}$ is about 1.5 times larger. Mizukami et al. (2001a, 2001b) also reported FMR measurements on $\mathrm{Ta} \mid \mathrm{Py}$ sandwiches that had only a small damping enhancement with respect to the bulk value.

According to earlier arguments, the absence of a significant thickness dependence of the damping in the $\mathrm{Cu} \mid \mathrm{Py}$ system could correspond to an unrealistically small conductance $g_{r}^{\uparrow \downarrow}$. The explanation should, however, be sought in the long spin-flip relaxation times in clean $\mathrm{Cu}$ (Meservey and Tedrow, 1978). Since the accumulated spins drive a diffusion spin current opposite to the pumping current, the additional damping vanishes in clean $\mathrm{Cu}$. On the other hand, $\mathrm{Pd}$ and especially $\mathrm{Pt}$ (which is below $\mathrm{Pd}$ in the periodic table) have much larger spin-relaxation rates, which can be rationalized by the higher atomic number and complex Fermi surfaces [Pd appears to also have an additional spin-decoherence mechanism due to spin-flip scattering at magnetization fluctuations; see Foros, Woltersdorf, et al. (2005)]. We note that the spin-flip efficiency of a dirty normal metal is determined by defects and impurities as well. Lubitz et al. (2003) reported a significant damping contribution that scaled as $1 / d$ as a function of the Fe-layer thickness $d$ in polycrystalline $\mathrm{Cu}|\mathrm{Fe}| \mathrm{Cu}$ sandwiches in contrast to the experiments by Mizukami et al. (2001b) on $\mathrm{Cu}|\mathrm{Py}| \mathrm{Cu}$. This was interpreted in terms of a larger $\mathrm{Cu}$ spin-relaxation rate in the $\mathrm{Fe} \mid \mathrm{Cu}$ as compared to the $\mathrm{Cu} \mid \mathrm{Py}$ system. The effect of spin flip in the normal metal on the excess damping in multilayers is treated quantitatively in the next subsection.

Ingvarsson et al. (2002) also carried out FMR studies on Py films sandwiched with normal metals $\mathrm{Pt}, \mathrm{Nb}$ (which is above $\mathrm{Ta}$ in the periodic table), and $\mathrm{Cu}$, as well as by insulators. Pt|Py combinations displayed a significantly stronger damping than other structures. They also identified an additional thickness-dependent damping correlated with the disorder in the Py films that was not reported by Mizukami et al. (2001a, 2001b). Damping processes that are intrinsic to the ferromagnetic layer cannot be addressed by the present theory, however.

Lagae et al. (2005) studied pulsed dynamics of a thin Py layer in contact with $\mathrm{Cu}$ or Ta layers. In addition, they investigated the role of a second, pinned ferromagnetic layer attached to the free layer via a $\mathrm{Cu}$ spacer or a tunnel barrier. The measured Gilbert damping was found to be consistent with the spin-pumping picture. We shall discuss the effect of the second ferromagnetic layer in Sec. IV.C.

Finally, we comment on the role of electron correlations in the spin-pumping formalism; see also Sec. VII.D. In order to calculate the mixing conductance, the scattering matrix has been obtained by Xia et al. (2002) and Zwierzycki et al. (2005) for the effective potential from Kohn-Sham density-functional theory. This poten- 
tial is calculated self-consistently and includes electronelectron interaction effects via the Hartree potential and an exchange-correlation potential in the local-spindensity approximation. The interface parameters, when computed self-consistently and nonperturbatively, include the magnetic moments induced in the normal metal by the proximity to the ferromagnet [also discussed by Šimánek (2003)]. For $\mathrm{Cu}$ and $\mathrm{Au}$ normalmetal films, this effect is very small due to the small densities of states at the Fermi energy. The question arises as to whether larger effects may be expected for materials such as $\mathrm{Pd}$ and $\mathrm{Pt}$ with a large Fermi-level density of states (see Table II). These metals are known to be "almost ferromagnetic" with a Stoner-enhanced spin susceptibility $\chi^{/} \chi_{0}=\left(1-\mathcal{N} I_{\mathrm{xc}}\right)^{-1}$, also listed in Table II. So, can the increased damping of the magnetization dynamics in contact with thin layers of Ta, Pd, and Pt as compared to $\mathrm{Cu}$ (Mizukami et al., 2001a, 2001b) be connected to their larger Fermi-level densities of states? In spin-pumping theory, the quantity that governs the damping enhancement is the mixing conductance. For metallic structures, band-structure calculations give results that are close to the Sharvin conductance of the normal metal, Eq. (64), thus not sensitive to the magnetic moments induced in the proximity to the ferromagnet. The Sharvin conductance for $\mathrm{Cu}, \mathrm{Ta}, \mathrm{Pd}$, and $\mathrm{Pt}$ is seen to change less than $\mathcal{N}$. More significantly, the trend does not correspond to that observed experimentally for the damping enhancement. The Sharvin conductance is maximal for Ta, not Pd with the highest spin susceptibility, supporting our earlier conclusion that spin dissipation in the normal conductors is crucial for the enhanced magnetization damping, as is explored in more detail below.

\section{B. Diffuse systems}

In the previous subsection, we have concentrated on the extreme situation in which the normal-metal layer is an ideal spin sink that immediately absorbs the injected spin current $\mathbf{I}_{s}^{\text {pump }}$, by either relaxation through spin-flip processes or the absence of backscattering. Disregarding $\mathbf{I}_{s}^{(0)}$, the total spin current through the contact, Eq. (57), reduces to $\mathbf{I}_{s}=\mathbf{I}_{s}^{\text {pump }}$. In general, we have to take into account the spin accumulation in a diffuse normal metal that drives a spin current $\mathbf{I}_{s}^{(0)}$ back into the ferromagnet. As a technical note, we recall that in this regime the drift correction (34) should be applied to the conductance parameters entering Eqs. (25), (26), and (38).

As the simplest example, we first discuss a $F \mid N$ bilayer, in which a magnetization precession generates a pure spin but no charge current. The spin accumulation or nonequilibrium chemical-potential imbalance $\boldsymbol{\mu}_{s}(x)$ [similar to Eq. (16), but with spatial dependence] in the normal metal is a vector with magnitude that depends on the distance from the interface $x, 0<x<L$, where $L$ is the thickness of the normal-metal film (see Fig. 6). When the ferromagnetic magnetization steadily precesses (in an easy plane) around the $z$ axis, $\mathbf{m} \times \mathbf{m}$ and

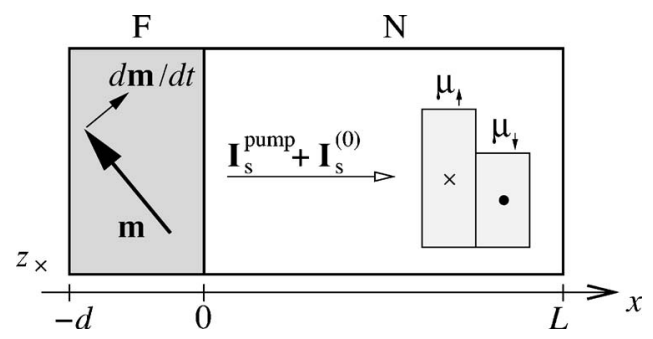

FIG. 6. Schematic view of a $F \mid N$ bilayer in which the magnetization direction $\mathbf{m}(t)$ of the ferromagnet $F$ rotates in an easy plane, pumping a spin current $\mathbf{I}_{s}^{\text {pump }}$ into the adjacent normalmetal layer $N$. The $N$ layer here is not an ideal reservoir but rather a film of the same cross section as the magnetic layer $F$. The spin pumping builds up a position $(x)$ dependent spin accumulation in $N$ that either relaxes by spin-flip scattering or drives a spin current back into the ferromagnet as $\mathbf{I}_{s}^{(0)}$.

the normal-metal spin accumulation $\boldsymbol{\mu}_{s}(x)$ are oriented along $z$, as depicted in Fig. 6. We shall see in the following that the instantaneous $\boldsymbol{\mu}_{s}$ is always perpendicular to $\mathbf{m}$ even in the case of a precessing ferromagnet with time-dependent rotation axis, as long as the precession frequency $\omega$ is smaller than the spin-flip rate $\tau_{\mathrm{sf}}^{-1}$ in the normal metal.

In the frequency domain, a spin accumulation diffuses in a normal metal according to (Johnson and Silsbee, 1988)

$$
i \omega \boldsymbol{\mu}_{s}=D \partial_{x}^{2} \boldsymbol{\mu}_{s}-\frac{\boldsymbol{\mu}_{s}}{\tau_{\mathrm{sf}}},
$$

where $D=v_{F}^{2} \tau / 3$ is the diffusion coefficient (in three dimensions) in terms of the Fermi velocity $v_{F}$ and the transport mean free time $\tau$. It is assumed here that the frequency $\omega$ is smaller than the scattering rate $\tau^{-1}$. The ratio of the momentum to spin-flip scattering time is an important parameter:

$$
\epsilon=\frac{\tau}{\tau_{\mathrm{sf}}} .
$$

Equation (65) holds when $\epsilon \ll 1$, i.e., the spin-flip relaxation may be treated perturbatively. The boundary conditions are given by the spin current $\mathbf{I}_{s}$ at the $F \mid N$ interface $x=0$ and vanishing of the spin current at the outer boundary $x=L$ :

$$
\begin{aligned}
& x=0: \partial_{x} \boldsymbol{\mu}_{s}=-\frac{2}{\hbar \mathcal{N S D}} \mathbf{I}_{s}, \\
& x=L: \partial_{x} \boldsymbol{\mu}_{s}=0,
\end{aligned}
$$

where $S$ is the interface area and $\mathcal{N}$ the normal-metal one-spin density of states per unit volume. The solution of Eqs. (65) and (67) is

$$
\boldsymbol{\mu}_{s}(x)=\frac{\cosh [\kappa(x-L)]}{\sinh \kappa L} \frac{2}{\hbar \mathcal{N S D} \kappa} \mathbf{I}_{s},
$$

where $\kappa=\lambda_{\text {sd }}^{-1} \sqrt{1+i \omega \tau_{\text {sf }}}$, recalling that $\lambda_{\text {sd }}=\sqrt{D \tau_{\text {sf }}}$ is the spin-diffusion length in the normal metal. In Sec. III.C 
we used similar arguments to calculate the spin accumulation generated by the precessing magnetization. Whereas in Sec. III.C the magnitude of the spin accumulation and its relevance for spintronics are discussed, we focus here on the effect of the spin accumulation on ferromagnetic magnetization dynamics.

We assume in the following that the precession frequency $\omega$ is smaller than the spin-flip relaxation rate, $\omega$ $\ll \tau_{\text {sf }}^{-1}$, so that $\kappa \approx \lambda_{\text {sd }}^{-1}$. For a typical effective field of $1 \mathrm{~T}$, $\omega \sim 10^{11} \mathrm{~s}^{-1}$. The scattering rate corresponding to a mean free path of $\lambda \sim 10 \mathrm{~nm}$ is $\tau^{-1} \sim 10^{14} \mathrm{~s}^{-1}$. Consequently, for such metals, $\omega \ll \tau_{\text {sf }}^{-1}$ requires $\epsilon \geq 10^{-3}$. This condition is easily satisfied (Meservey and Tedrow, 1978) for impurities with higher atomic numbers $Z$ [since $\epsilon$ scales as $Z^{4}$ (Abrikosov and Gor'kov, 1962)]. The highfrequency limit $\omega \gtrsim \tau_{\mathrm{sf}}^{-1}$, on the other hand, is relevant for systems with weak spin-flip scattering in the normal metal and has been discussed in the context of the spinbattery concept in Sec. III.C. We shall see that a sizable Gilbert damping enhancement requires a large spin-flip probability $\epsilon \gtrsim 10^{-1}$, thus $\omega \ll \tau_{\text {sf }}^{-1}$, unless the frequency is comparable to the momentum-scattering rate in the normal metal. [The latter regime lies below our treatment based on Eqs. (65) and (67).]

It is convenient to define an effective energy-level spacing of states participating in spin-flip scattering events in a thick (i.e., $L \gg \lambda_{\mathrm{sd}}$ ) normal-metal film as

$$
\delta_{\text {sd }}=\frac{1}{\mathcal{N} S \lambda_{\text {sd }}}
$$

The spin-diffusion length (which sets the scale for spinaccumulation penetration into the normal layer) written in terms of the relevant scattering times is

$$
\lambda_{\mathrm{sd}}=v_{F} \sqrt{\frac{\tau \tau_{\mathrm{sf}}}{3}} .
$$

The spin-accumulation-driven spin current $\mathbf{I}_{s}^{(0)}$ through the interface is obtained by substituting $\boldsymbol{\mu}_{s}(x=0)$ from Eq. (68) into Eq. (26) to give

$$
\mathbf{I}_{s}=\mathbf{I}_{s}^{\text {pump }}+\mathbf{I}_{s}^{(0)}=\mathbf{I}_{s}^{\text {pump }}-\beta\left(\tilde{g}_{r}^{\uparrow \downarrow} \mathbf{I}_{s}+\tilde{g}_{i}^{\uparrow \downarrow} \mathbf{I}_{s} \times \mathbf{m}\right),
$$

where the spin current returning into the ferromagnet is governed by the "backflow factor" $\beta$ :

$$
\beta=\frac{\tau_{\mathrm{sf}} \delta_{\mathrm{sd}} / h}{\tanh \left(L / \lambda_{\mathrm{sd}}\right)} .
$$

The last equality in Eq. (71) is obtained from Eq. (26) by assuming that the nonequilibrium transport is limited to spin currents that are polarized normal to the magnetization. This is allowed in the adiabatic regime when $\omega$ $\ll \tau_{\text {sf }}$ where the average angular momentum of the pumped and backscattered spins is at a given instant perpendicular to the magnetization. Consequently, as long as the ferromagnetic spin-relaxation length is larger than the transverse spin-coherence length $\lambda_{\mathrm{sc}}$, we may disregard spin relaxation inside the ferromagnet. $\beta$ is given by the ratio between the energy-level spacing of the normal-metal film with a thickness $L_{\mathrm{sf}}=\min \left(L, \lambda_{\mathrm{sd}}\right)$ and the spin-flip rate. When the normal metal is shorter than its spin-flip diffusion length, $L \ll \lambda_{\mathrm{sd}}, \beta \rightarrow \tau_{\mathrm{sf}} \delta / h$, where $\delta=(\mathcal{N S} L)^{-1}$ is the energy-level splitting. In the opposite regime of thick normal metals, $L \gg \lambda_{\text {sd }}, \beta$ $\rightarrow \tau_{\mathrm{sf}} \delta_{\mathrm{sd}} / h$.

By solving Eq. (71), the total spin current $\mathbf{I}_{s}$ can be expressed in terms of the pumped spin current $\mathbf{I}_{s}^{\text {pump }}$, Eq. (38), to finally obtain

$$
\mathbf{I}_{s}=\frac{\hbar}{4 \pi}\left(\operatorname{Re} \mathcal{A}_{\mathrm{eff}}^{\uparrow \downarrow} \mathbf{m} \times \frac{d \mathbf{m}}{d t}+\operatorname{Im} \mathcal{A}_{\mathrm{eff}}^{\uparrow \downarrow} \frac{d \mathbf{m}}{d t}\right),
$$

which has the same form as Eq. (38), but with effective spin-pumping efficiencies $\mathcal{A}_{\text {eff }}^{\uparrow \downarrow}$ :

$$
\frac{1}{\mathcal{A}_{\mathrm{eff}}^{\uparrow \downarrow}}=\frac{1}{\tilde{g}^{\uparrow \downarrow}}+\frac{R_{\mathrm{sd}}}{\tanh \left(L / \lambda_{\mathrm{sd}}\right)} .
$$

Here, $R_{\mathrm{sd}}=\tau_{\mathrm{sf}} \delta_{\mathrm{sd}} / h$ is the dimensionless resistance [in units of $\left.\left(2 e^{2} / h\right)^{-1}\right]$ of the normal-metal layer of thickness $\lambda_{\text {sd }}$, which follows from Eq. (69) and the Einstein relation $\sigma=e^{2} D \mathcal{N}$ between conductivity $\sigma$ and diffusion coefficient $D$. When $L \gg \lambda_{\text {sd }}$, the effective spin pumping out of the ferromagnet is governed by the mixing conductance of the $F \mid N$ interface in series with a diffusive normal-metal film of thickness $\lambda_{\text {sd }}$.

By accepting the approximation (62), we disregard $\tilde{g}_{i}^{\uparrow \downarrow}$ in the remainder of this section. $\operatorname{Im} \mathcal{A}_{\text {eff }}^{\uparrow \downarrow}$ then also vanishes and upon substitution into Eq. (14) the damping torque due to the spin current $\mathbf{I}_{s}$ obeys the Gilbert phenomenology. The effective Gilbert damping parameter in the diffuse model reads [cf. Eq. (63)]:

$$
G_{\mathrm{eff}}-G=\left[1+\tilde{g}_{r}^{\uparrow \downarrow} \frac{R_{\mathrm{sd}}}{\tanh \left(L / \lambda_{\mathrm{sd}}\right)}\right]^{-1} \frac{\hbar \gamma^{2} \tilde{g}_{r}^{\uparrow \downarrow}}{4 \pi V} .
$$

The prefactor on the right-hand side of Eq. (75) reflects the reduction effect on the Gilbert damping that is caused by spin diffusion back into the ferromagnet. This correction has been disregarded in Sec. IV.A where normal metals were considered to be ideal spin sinks. Because spins accumulate in the normal metal polarized transversely to the ferromagnetic magnetization, the spin-accumulation-driven transport across the $F \mid N$ contact is governed by the spin-mixing conductance (just as the spin-pumping current). The absence of spin accumulations or currents polarized collinear to the magnetization (in the limit $\omega \ll \tau_{\mathrm{sf}}^{-1}$ ) explains why the diagonal components of the conductance matrix $\tilde{g}^{\sigma \sigma^{\prime}}$ do not enter into Eq. (75).

The numerical values of the parameters in Eq. (75) can be estimated with the free-electron model for the normal metal, for which $\mathcal{N}=k_{F}^{2} / \pi h v_{F}$. Using Eqs. (70) and (69), we find $R_{\mathrm{sd}}^{-1}=h / \delta_{\mathrm{sd}} \tau_{\mathrm{sf}}=4 \sqrt{\epsilon / 3} g_{N}^{\mathrm{Sh}}$, where $g_{N}^{\mathrm{Sh}}$ $=S k_{F}^{2} / 4 \pi$ is the dimensionless Sharvin conductance (number of transport channels) of the normal metal. Using the approximation (64) for the mixing conductance $g_{r}^{\uparrow \downarrow}$, and Eq. (33) to correct for the drift contribution, we obtain $\tilde{g}_{r}^{\uparrow \downarrow} \approx 2 g_{N}^{\mathrm{Sh}}$. We thus estimate 


$$
G_{\mathrm{eff}}-G \sim \frac{\hbar \gamma^{2} \tilde{g}_{r}^{\uparrow \downarrow} / 4 \pi V}{1+\left[\sqrt{\epsilon} \tanh \left(L / \lambda_{\mathrm{sd}}\right)\right]^{-1}} .
$$

A significantly increased damping therefore requires a high spin-flip to spin-conserving scattering probability, $\epsilon \gtrsim 10^{-2}$, and sufficiently thick normal layers, $L \gtrsim \lambda_{\text {sd }}$ ( $L$ $\gtrsim \lambda_{\text {sd }}$ alone is not a sufficient condition, however, contrary to what has sometimes been assumed in the literature). Poor spin sinks that do not modify the magnetization dynamics are characterized by a large denominator in Eq. (76). Clean light metals with atomic number $Z \lesssim 50$, such as $\mathrm{Al}, \mathrm{Cr}$, and $\mathrm{Cu}$, as well as heavier metals with only $s$ electrons in the conduction band, such as Ag, are less effective spin sinks because of a relatively small intrinsic spin-orbit coupling, typically in the range of $\epsilon \lesssim 10^{-2}$ (Meservey and Tedrow, 1978; Bergmann, 1982; Yang et al., 1994). Heavier elements with $Z \gtrsim 50$ and $p$ or $d$ character of the conduction electrons, such as $\mathrm{Pd}, \mathrm{Pt}$, and $\mathrm{Pb}$, are effective spin sinks with much larger $\epsilon \gtrsim 10^{-1}$ (Meservey and Tedrow, 1978). Au is intermediate in $\epsilon$ (Chiang et al., 2004), presumably due to the $s$ character of its outer orbital. These conclusions explain the hierarchy of the observed Gilbert damping enhancement observed by Mizukami et al. (2001a, 2001b); see Sec. IV.A. By doping a small- $\epsilon$ matrix with high $Z$ or magnetic impurities (e.g., $\mathrm{Cu}$ lattice with $\mathrm{Pt}$ atoms), a good spin sink can be created. We conclude that the damping parameter (and thus the switching speed) of thin ferromagnetic films can be engineered flexibly by coating them with suitable normal metals.

In the limit of a large ratio of the spin-flip to momentum scattering, $\epsilon \sim 1$, the spin-diffusion equation and, consequently, Eq. (76) do not hold. In this regime, the normal metal efficiently relaxes the spins injected from the ferromagnet. Foros, Woltersdorf, et al. (2005) investigated this scenario for $\mathrm{Pd}$ (in contact with $\mathrm{Fe}$ ) which appears to have a spin-relaxation rate, arguably by paramagnons, that is faster than the bulk momentumscattering rate. When the spin-decoherence length $v_{F} \tau_{\text {sf }}$ is thicker, ballistic Pd films should otherwise be good spin sinks. The ferromagnetic relaxation by ideal spin sinks is determined by the bare spin-mixing conductance $g^{\uparrow \downarrow}$, as discussed in Sec. IV.A. The latter generally provides an upper bound for the magnitude of additional Gilbert damping. However, in the present theory, spinorbit coupling is treated phenomenologically in the diffuse-transport regime only. Strong spin-orbit coupling immediately at interfaces, for example, requires generalization of spin-pumping and circuit theories beyond the scope of this review.

Infinite versus vanishing spin-flip rates in the normal metal are two extremes for $F \mid N$ bilayer dynamics. In the former case, the damping parameter $G_{\text {eff }}$ is enhanced, and in the latter case unmodified. Both limits are experimentally accessible by using $\mathrm{Pt}$ as a good or $\mathrm{Cu}$ as a poor spin sink, as shown by Mizukami et al. (2001a, 2001b) for $N|\mathrm{Py}| N$ sandwiches.

Experiments on Py where both $\mathrm{Cu}$ and Pt were combined in a $\mathrm{Py}|\mathrm{Cu}| \mathrm{Pt}$ heterostructure (Mizukami et al.,

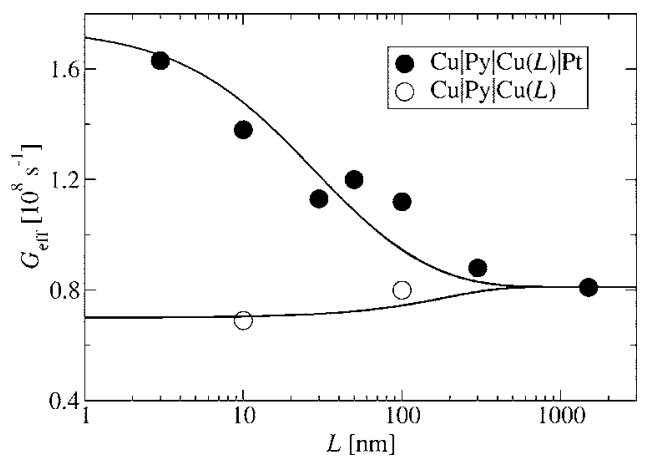

FIG. 7. Gilbert damping constants (circles) in $\mathrm{Cu}|\mathrm{Py}(3 \mathrm{~nm})| \mathrm{Cu}(L) \mid \mathrm{Pt}(5 \mathrm{~nm})$ and $\mathrm{Cu}|\mathrm{Py}(3 \mathrm{~nm})| \mathrm{Cu}(L)$ structures measured by Mizukami et al. (2002a, 2002b) as a function of Cu-layer thickness $L$. Solid lines are the theoretical results according to Eq. (80) with parameters discussed in the text. The horizontal axis uses a logarithmic scale.

2002a, 2002b) allowed for studying spin diffusion in the central $\mathrm{Cu}$ layer. The measured room-temperature magnetization damping in $\mathrm{Cu}|\mathrm{Py}| \mathrm{Cu}(L)$ and $\mathrm{Cu}|\mathrm{Py}| \mathrm{Cu}(L) \mid \mathrm{Pt}$ structures as a function of $\mathrm{Cu}$-film thickness $L$ is shown by circles in Fig. 7. The experiments can be understood by a slight extension of the diffusion-theory-based discussion for $F \mid N$ bilayers. The damping is governed by the angular-momentum loss of the ferromagnet through the normal-metal compound $N_{1} \mid N_{2}$, as schematically shown in Fig. 8. Once injected into $N_{1}$, spins are either scattered back into the ferromagnet or relax in $N_{1}$ or $N_{2}$. When there is only a very weak spin-flip scattering in $N_{1}$ compared to $N_{2}$, the spins have to diffuse through $N_{1}$ before they can relax in the second normal-metal layer $N_{2}$. For simplicity, we model $N_{2}$ as an ideal sink that instantaneously relaxes incoming spins, as is appropriate for Pt. The analysis below illustrates that the ferromagnetic magnetization damping as a function of $L$ provides important information about spin transport through $N_{1}$ and the $N_{1} \mid N_{2}$ interface. The $\mathrm{Cu}$ substrate on the other side of the Permalloy film (Mizukami et al., 2002a, 2002b) can be disregarded since it is a poor spin sink.

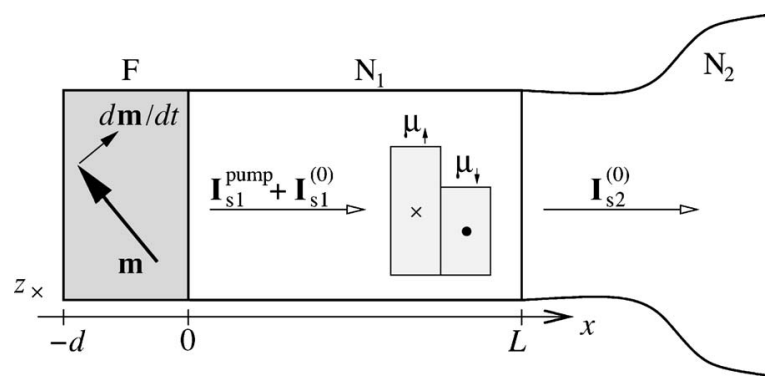

FIG. 8. Similar to Fig. 6, but the normal-metal system is a $N_{1} \mid N_{2}$ bilayer. Ferromagnetic magnetization precession pumps spins into the first normal-metal layer $N_{1}$. The spin accumulation in $N_{1}$ either flows back into the ferromagnet $F$ as spin current $\mathbf{I}_{s 1}^{(0)}$, relaxes in $N_{1}$, or leaves via the second normalmetal layer $N_{2}$ as spin current $\mathbf{I}_{s 2}^{(0)}$. The spin accumulation in the ideal spin sink $N_{2}$ vanishes. 
Similar to Eqs. (67), the boundary conditions for the spin accumulation in the normal metal $N_{1}$ are now

$$
\begin{aligned}
& x=0: \partial_{x} \boldsymbol{\mu}_{s}=-\frac{2}{\hbar \mathcal{N S} D} \mathbf{I}_{s 1}, \\
& x=L: \partial_{x} \boldsymbol{\mu}_{s}=-\frac{2}{\hbar \mathcal{N S} D} \mathbf{I}_{s 2} .
\end{aligned}
$$

$\mathbf{I}_{s 1}$ and $\mathbf{I}_{s 2}$ are the total spin currents through the left $(x=0)$ and right $(x=L)$ interfaces, respectively. $\mathbf{I}_{s 1}$ $=\mathbf{I}_{s 1}^{\text {pump }}+\mathbf{I}_{s 1}^{(0)}$, cf. Eq. (71) in the previous subsection, is the sum of the pumped, Eq. (38), and the spinaccumulation-driven, Eq. (26), spin currents. $\mathbf{I}_{s 2}$, on the other hand, is entirely governed by the $N_{1} \rightarrow N_{2}$ diffusion

$$
\mathbf{I}_{s 2}=\frac{\tilde{g}}{4 \pi} \boldsymbol{\mu}_{s}(x=L)
$$

where $\tilde{g}$ is the effective spin conductance of the $N_{1} \mid N_{2}$ interface:

$$
\frac{1}{\tilde{g}}=\frac{1}{g_{N_{1} \mid N_{2}}^{\sigma \sigma}}-\frac{1}{2 g_{N_{1}}^{\mathrm{Sh}}} .
$$

Here, we have corrected the bare single-spin resistance $1 / g_{N_{1} \mid N_{2}}^{\sigma \sigma}$ of the all-normal interface for the drift effect by subtracting the Sharvin contribution on the $N_{1}$ side only because the layer $N_{2}$ is assumed to be an ideal spin bath. Solving the diffusion equation (65) with the boundary conditions (77), we find the spin current $\mathbf{I}_{s 1}$, which is transferred to the magnetization as a torque, as discussed in the preceding discussion for the bilayer. The Gilbert damping enhancement due to the spin relaxation in the trilayer is then found to be

$$
G_{\mathrm{eff}}-G=\left[1+\tilde{g}_{r}^{\uparrow \downarrow} R_{\mathrm{sd}} \frac{1+\tanh \left(L / \lambda_{\mathrm{sd}}\right) \tilde{g} R_{\mathrm{sd}}}{\tanh \left(L / \lambda_{\mathrm{sd}}\right)+\tilde{g} R_{\mathrm{sd}}}\right]^{-1} \frac{\hbar \gamma^{2} \tilde{g}_{r}^{\uparrow \downarrow}}{4 \pi V},
$$

where $\lambda_{\text {sd }}$ and $R_{\text {sd }}$ parametrize the spin diffusion in $N_{1}$, $\tilde{g}_{r}^{\uparrow \downarrow}$ is the renormalized mixing conductance (33) of the $F \mid N_{1}$ interface, and $\tilde{g}$ is the spin-transfer conductance (79) of the $N_{1} \mid N_{2}$ interface.

Setting $\tilde{g}=0$ decouples the two normal-metal systems and reduces Eq. (80) to Eq. (75), the damping coefficient of the $F \mid N_{1}$ bilayer. In the experiment by Mizukami et al. (2002a, 2002b), the Permalloy thickness $d=3 \mathrm{~nm}$ was fixed and the $\mathrm{Cu}$ film thickness $L$ was varied between 3 and $1500 \mathrm{~nm}$ as shown by the circles in Fig. 7. The theoretical result (80) is plotted in Fig. 7 for comparison, using the following parameters: the bulk damping $G$ $=0.7 \times 10^{8} \mathrm{~s}^{-1}$ (Patton et al., 1975; Bastian and Biller, 1976), the spin-flip probability $\epsilon=1 / 700$, and the spindiffusion length $\lambda_{\mathrm{sd}}=250 \mathrm{~nm}$ for $\mathrm{Cu}$ (which correspond to the mean free path $\lambda=\sqrt{3 \epsilon} \lambda_{\text {sd }}=16 \mathrm{~nm}$ ), in satisfactory agreement with values reported in the literature (Meservey and Tedrow, 1978; Yang et al., 1994; Jedema et al., 2001), $\tilde{g}_{r}^{\uparrow \downarrow} / S=16 \mathrm{~nm}^{-2}$ extracted from the experi- mental angular magnetoresistance of $\mathrm{Py} \mid \mathrm{Cu}$ (Bauer et al., $2003 \mathrm{~b}$ ), and $\tilde{g} / S=35 \mathrm{~nm}^{-2}$ for the $\mathrm{Cu} / \mathrm{Pt}$ contact. This $\tilde{g}$ corresponds to the bare one-spin conductance $g^{\sigma \sigma} / S$ $=16 \mathrm{~nm}^{-2}$, which is close to the Sharvin conductance of $\mathrm{Cu}$ (see Table I). Figure 7 shows a satisfactory agreement (within the experimental error) between experiments and theory. This proves the diffusive nature of spin transfer in the $\mathrm{Cu}$ spacer. Whereas the detailed mechanism for spin injection (relaxation) at the $\mathrm{Py} \mid \mathrm{Cu}$ $(\mathrm{Cu} \mid \mathrm{Pt})$ interface cannot be deduced directly, the agreement on absolute scale obtained with parameters taken from other sources strongly supports the spin-pumping picture.

It is illuminating to discuss Eq. (80) in the limit of vanishing spin flip in the spacer layer $N_{1}$. Recalling the definitions for $\lambda_{\text {sd }}$, Eq. (70), and $\delta_{\text {sd }}$, Eq. (69), and taking the limit $\tau_{\mathrm{sf}} \rightarrow \infty$, we find that Eq. (80) reduces to Eq. (63), only with $2 g^{\uparrow \downarrow}$ (where the factor of 2 corresponds to two $F \mid N$ interfaces in the $N|F| N$ trilayers) replaced by $g_{\text {eff }}^{\uparrow \downarrow}$

$$
\frac{1}{g_{\text {eff }}^{\uparrow \downarrow}}=\frac{1}{\tilde{g}^{\uparrow \downarrow}}+R_{N_{1}}+\frac{1}{\tilde{g}},
$$

where $R_{N_{1}}=\left(2 e^{2} / h\right) L / S \sigma$ is the dimensionless resistance of the $N_{1}$ layer with conductivity $\sigma$. The right-hand side of Eq. (81) is simply the inverse bare mixing conductance of the diffuse $N_{1}$ spacer in series with its two interfaces, one with $F$ and the other with $N_{2}$ (Bauer et al., 2003b). In particular, when $N_{1}$ is thick enough, the total mixing conductance $g_{\text {eff }}^{\uparrow \downarrow}$ is limited by the spacer separating $F$ and $N_{2}$ (Brataas et al., 2000, 2001). The spin pumping into layer $N_{1}$ with subsequent spin-conserving diffusion and then spin absorption by the ideal spin sink $N_{2}$ is thus equivalent to spin pumping (38) across an effective scatterer separating the ferromagnet $F$ from the ideal spin sink $N_{2}$.

The general trends in Fig. 7 can be understood as follows. Since $\mathrm{Cu}$ is a poor spin sink, a $\mathrm{Py} \mid \mathrm{Cu}$ contact with a single $\mathrm{Cu}$ film only weakly increases the damping for all thicknesses. This enhancement saturates at $L \gg \lambda_{\text {sd }}$ and vanishes in the limit $L \ll \lambda_{\text {sd }}$. When a Pt film, a very good spin sink, is connected to the bilayer and the $\mathrm{Cu}$ spacer is thinner than the transport mean free path, $L$ $\ll \lambda$, the spin accumulation is uniform throughout the $\mathrm{Cu}$. The spin pumping is then partitioned. One fraction of the pumped spins is reflected back into the ferromagnet, while the remainder is transmitted to and subsequently relaxes in the $\mathrm{Pt}$ layer. Their ratio equals the ratio between the conductance $\tilde{g}_{r}^{\uparrow \downarrow}$ of the Py|Cu and the conductance $\tilde{g}$ of the $\mathrm{Cu} \mid \mathrm{Pt}$ interfaces, and is of the order of unity. Since a significant fraction of the spin-pumping current is dissipated in $\mathrm{Pt}$, a large magnetization damping is achieved. When $L$ is increased, less spins manage to diffuse through the $\mathrm{Cu}$ spacer, and, in the limit $L$ $\gg \lambda_{\text {sd }}$, a majority of the spins scatter back into the ferromagnet before sensing the presence of the Pt layer. In the intermediate regime, the spin pumping into the $\mathrm{Pt}$ layer decays algebraically on the scale of the transport 


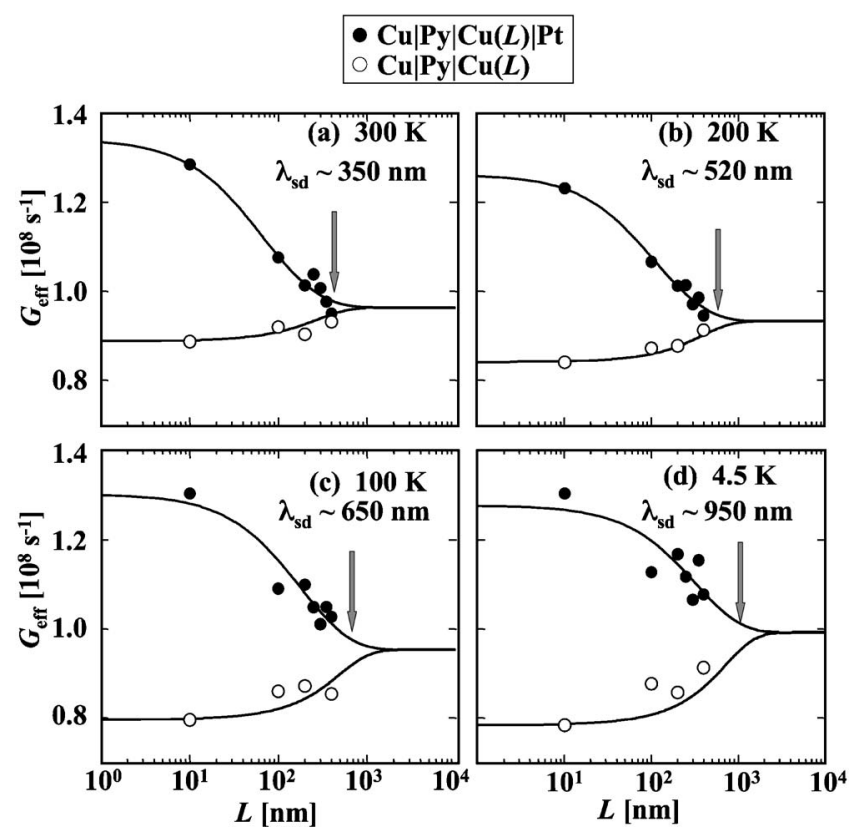

FIG. 9. Gilbert damping measured in $\mathrm{Cu} \mid \mathrm{Py}(3$ $\mathrm{nm})|\mathrm{Cu}(L)| \operatorname{Pt}(2 \mathrm{~nm})$ and $\mathrm{Cu}|\mathrm{Py}(3 \mathrm{~nm})| \mathrm{Cu}(L)$ structures for several $\mathrm{Cu}$-layer thicknesses $L$ at various temperatures. Solid lines are fits based on spin-pumping theory. Adapted from Yakata et al., 2005.

mean free path and exponentially on the scale of the spin-diffusion length.

The temperature dependence of the Gilbert damping in such trilayers has been measured recently by the same group (Yakata et al., 2005); see Fig. 9. The spin-diffusion length extracted from the data by means of an analysis similar to the one presented here increases as the temperature is lowered, indicating a reduced role of phonon scattering at low temperatures. The good agreement with results from electrical-transport experiments by Jedema et al. (2001) establishes FMR experiments as an important tool for measuring spin transport in magnetic heterostructures.

The dependence of the damping on the $\mathrm{Cu}$-layer thickness $L$ in the $\mathrm{Cu}|\mathrm{Py}| \mathrm{Cu}(L) \mathrm{Pt}$ multilayers reflects the amount of accumulation in the normal metals. This spin accumulation, in turn, indicates that an excited ferromagnet (as in the FMR experiment discussed here) transfers spins into adjacent nonmagnetic layers according to the spin pumping (38). The concept of the spin battery discussed in Sec. III.C relies on this effect.

\section{Enhanced Gilbert damping in spin valves: First-principles calculations versus experiment}

In $F|N| F$ structures, the presence of two ferromagnetic layers can make damping possible for each individual layer even in the absence of spin-flip relaxation in the system (Berger, 1996). The point is that if one ferromagnet is excited while the other is static, the latter acts as a sink for transverse spin currents pumped by the former. In this subsection, we assume a sufficiently thick or dis- ordered normal spacer, so that it does not support any persistent spin currents or, in other words, the static exchange interaction between the magnetic films vanishes (see Sec. I.B). Static exchange will be taken into account in Sec. $\mathrm{V}$ in discussing coupled dynamics of two or more magnetic films. If the $F|N| F$ magnetic structure is weakly excited from a collinear equilibrium state, then by defining the effective (complex-valued) spin-pumping efficiency $\mathcal{A}_{F|N| F}^{\uparrow \downarrow}$ by

$$
\frac{1}{\mathcal{A}_{F|N| F}^{\uparrow \downarrow}}=\frac{1}{\tilde{g}_{1}^{\uparrow \downarrow}}+\frac{2 e^{2}}{h} \frac{L}{S \sigma}+\frac{1}{\tilde{g}_{2}^{\uparrow \downarrow}},
$$

we can summarize with an analysis similar to that of the preceding section as follows. The total spin current $\mathbf{I}_{s}$ through the normal spacer consisting of the pumped and backflow components, Eq. (36), is given by the righthand side of Eq. (38) with $\mathcal{A}^{\uparrow \downarrow}$ replaced by $\mathcal{A}_{F|N| F}^{\uparrow \downarrow}$, where $\mathbf{m}$ is the magnetization of the excited film (assuming one of the ferromagnets is static). The effective damping constant and gyromagnetic ratio are then given by Eqs. (59) and (60) with $\mathcal{A}_{F|N| F}^{\uparrow \downarrow}$ substituted for $\mathcal{A}^{\uparrow \downarrow}+\mathcal{A}^{\prime \uparrow \downarrow}$. In Eq. (82), $\sigma$ is the conductivity of the $N$ spacer, $L$ is its thickness, and $S$ is the area of the trilayer. In the spirit of the theory discussed in Sec. II.B, Eq. (82) requires that the spacer (in series with the two interfaces) be diffuse. The transmission mixing conductance $t^{\uparrow \downarrow}$ is disregarded, assuming sufficiently thick magnetic films, $d \gg \lambda_{\mathrm{sc}}$, or insulating substrate and cap for the $F|N| F$ trilayer. Adding inverse spin-mixing conductances in series with the diffuse-spacer resistance in Eq. (82) reflects partitioning of the pumped spin currents between the two magnetic layers, having disregarded spin relaxation in the spacer.

Urban et al. (2001) reported room-temperature observations of increased Gilbert damping for a system consisting of two epitaxially grown Fe layers separated by a $\mathrm{Au}$ spacer layer. The complete structures were $\mathrm{GaAs}|(8,11,16,21,31) \mathrm{Fe}| 40 \mathrm{Au}|40 \mathrm{Fe}| 20 \mathrm{Au} \mid(001)$, where the integers represent the number of monolayers, and the samples differ in the thickness of the thinner Fe film. The interface magnetic anisotropies allowed Urban et al. (2001) to separate the FMR fields of the two Fe layers with resonance-field differences that can exceed 5 times the FMR linewidths. Hence the FMR measurements for thinner $F$ layer can be carried out with a nearly static thick layer. The FMR linewidth of the thin $F$ layer increases in the presence of the second layer. The difference in the FMR linewidths between the magnetic bilayer and single-layer structures is nearly inversely proportional to the thin-film thickness $d$, suggesting that the additional damping occurs due to its $F \mid N$ interface. Second, the additional linewidth is linearly dependent on microwave frequency for both the in-plane (the saturation magnetization parallel to the film surface) and perpendicular (the saturation magnetization perpendicular to the film surface) configurations, implying that the additional contribution to the FMR linewidth can be described strictly as an interface Gilbert damping (Urban et al., 2001). 


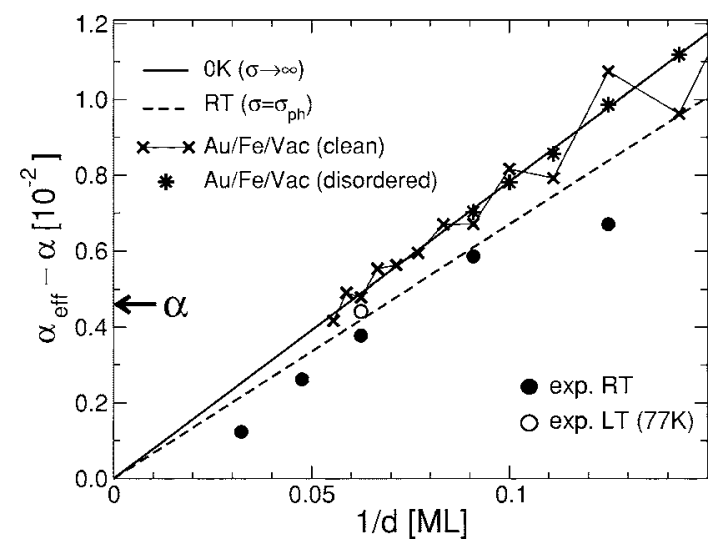

FIG. 10. Enhancement of the Gilbert damping coefficient for an $\mathrm{Fe}|\mathrm{Au}| \mathrm{Fe}$ trilayer as a function of $1 / d$ where $d$ is the thickness of the excited Fe layer in monolayers (ML's). The filled circles $(-)$ are the room-temperature values measured by Urban et al. (2001) and the open one $(\bigcirc)$ is a low-temperature (77 K) value from Heinrich, Urban, et al. (2003). The theoretical prediction based on Eq. (83) for $0 \mathrm{~K}$ (with $\sigma \rightarrow \infty$ ) is shown as solid line and the room-temperature (RT) corrected (with phonon scattering) one as dashed lines. The results of $0 \mathrm{~K}$ calculations for a $\mathrm{Au}|\mathrm{Fe}|$ vacuum system are given by crosses $(\mathbf{X})$ and stars $(*)$ for specular and disordered interfaces, respectively. The value of the Gilbert damping $\alpha$ for a single Fe film is marked with an arrow. From Zwierzycki et al., 2005.

The magnetization of the thin ferromagnetic layer precesses in the external magnetic field, while the other static magnetic layer acts as a spin sink. No modification of the damping coefficient was measured for $\mathrm{GaAs}|\mathrm{Fe}| \mathrm{Au}$ structures without a second Fe layer. The latter finding is consistent with the prediction given by Eq. (75) in the $L \ll \lambda_{\text {sd }}$ limit, well fulfilled for the thin $\mathrm{Au}$ films of Urban et al. (2001). In the presence of the second Fe layer, Eq. (82) should be used. Neglecting $\operatorname{Im} \mathcal{A}_{F|N| F}^{\uparrow}$ leads to $\gamma_{\text {eff }}=\gamma$ and the damping enhancement

$$
\alpha_{\text {eff }}-\alpha=\frac{\hbar \gamma \operatorname{Re} \mathcal{A}_{F|N| F}^{\uparrow \downarrow}}{4 \pi M_{S} d S},
$$

where $\alpha \approx 0.0046$ is the dimensionless damping measured for a single Fe layer. Using $\gamma=2.1 \mu_{B} / \hbar$ (Heinrich et al., 1987) and the values of the interface and Sharvin conductances from Table I, Eq. (83) is compared with the experimental data in Fig. 10 for various assumptions of $\sigma$ in Eq. (82). In the low-temperature limit and neglecting the residual resistivity of the Au layer, $\sigma \rightarrow \infty$, Eq. (83) yields the solid line which overestimates the damping enhancement compared with the measured results. Using finite values of $\sigma$ leads to lower values of $\mathcal{A}_{F|N| F}^{\uparrow \downarrow}$ and indeed it was found experimentally (Heinrich, Urban, et al., 2003) that lowering the temperature (increasing the conductivity) increases the damping by as much as about $20 \%$ (open circle in Fig. 10). If one uses the room-temperature conductivity due to phonon scattering in crystalline bulk $\mathrm{Au}, \sigma_{\mathrm{ph}}=0.45 \times 10^{8} \Omega^{-1} \mathrm{~m}^{-1}$, the dashed line is obtained which, as expected, is closer to the room-temperature measurements. Measurements of the sheet conductivity (Heinrich, Urban, et al., 2003) indicate that the Au layers used in the experiments have non-negligible residual resistances. [We note, however, that the conductivity entering Eq. (82) does not include the interfacial-scattering contribution; the measurement of the sheet conductivity therefore does not give us direct information about $\sigma_{\text {res. }}$.] Assuming, for example, $\sigma_{\text {res }} \approx \sigma_{\mathrm{ph}}$ would yield the $0 \mathrm{~K}$ (with $\sigma=\sigma_{\text {res }}$ ) and roomtemperature (with $\sigma^{-1}=\sigma_{\text {res }}^{-1}+\sigma_{\mathrm{ph}}^{-1}$ ) lines in the close vicinity of the measured points.

The theoretical results represented by the straight lines in Fig. 10 are based upon the asymptotic, singleinterface value of $g_{r}^{\uparrow \downarrow}$ for $\mathrm{Au} \mid \mathrm{Fe}$ from Table I. This approximation needs to be relaxed in order to study possible size-dependent corrections in thin films. To estimate the variation resulting from finite-size effects, Zwierzycki et al. (2005) carried out a series of calculations for a $\mathrm{Au}|\mathrm{Fe}|$ vacuum system, using vacuum instead of GaAs for simplicity as in the experiment by Urban et al. (2001). The mixing conductance of the other, $\mathrm{Fe} \mid \mathrm{Au}$, interface in Eq. (82) was kept at its asymptotic value (Table I). The calculated thickness $(d)$ dependent mixing conductance $g_{r}^{\uparrow \downarrow}$ was then converted into the Gilbert damping via Eq. (83). The results for perfect (specular) structures, marked in Fig. 10 with black crosses, exhibit oscillations of non-negligible amplitude near the asymptotic values given by the solid line (arbitrarily taking the low-temperature regime, i.e., $\sigma \rightarrow \infty$ for reference). Introduction of interface disorder (two monolayers of $50 \%-50 \%$ alloy) yields values for the damping (stars in Fig. 10) essentially averaged to the limit given by single-interface calculations of Table I.

Lubitz et al. (2003) also reported an increased magnetization damping in polycrystalline $\mathrm{Cu}|\mathrm{Fe}| \mathrm{Cu}|\mathrm{Fe}| \mathrm{Cu}$ multilayers as compared to $\mathrm{Cu}|\mathrm{Fe}| \mathrm{Cu}$ structures. The additional damping scaled as $1 / d$ with magnetic-film thickness $d$, but was considerably larger than that for epitaxially grown systems reported by Urban et al. (2001). Besides, this damping was rapidly reduced by increasing the thickness of the $\mathrm{Cu}$ spacer to only several nanometers, which was interpreted by Lubitz et al. (2003) to be possibly due to a very short spin-flip length in their $\mathrm{Cu}$. We note that, based on Eq. (82), this could be alternatively explained by a short elastic scattering length in polycrystalline $\mathrm{Cu}$. Lubitz et al. (2003) found a moderate increase by about $10 \%$ in the additional damping on lowering the temperature to $77 \mathrm{~K}$, which is roughly consistent with the decrease in the phonon contribution to the resistivity.

This subsection has demonstrated that direct firstprinciples calculations can produce values of the damping coefficient in the same range as those measured experimentally in good-quality structures. Moreover, by taking into account various other sources of scattering in the Au spacer and/or quantum-size effects, calculations can be brought into close agreement with observations. A more conclusive comparison with experiments would require detailed knowledge of the microscopic structure 
of the experimental system, which is currently not available.

\section{DYNAMIC EXCHANGE INTERACTION}

\section{A. Magnetic bilayers}

The ground-state energy (free energy at finite temperatures) of more than one magnetic layer embedded in a nonmagnetic medium depends on the relative orientation of the magnetic moments. This is the essence of the static exchange coupling discussed in Sec. I.B. Disorder scrambles ballistic electron paths connecting magnetic layers and exponentially suppresses the static interaction as a function of the nonmagnetic spacer thickness between magnetic layers and the inverse mean free path. We can picture the dynamic coupling in terms of a localized magnetic moment that suddenly changes its direction, thus creating a nonequilibrium local spin accumulation. The latter partly precesses in the local-moment effective field and partly diffuses away. Other magnetic moments at distances $L$ experience this spin accumulation after a time $t_{d}=L^{2} / D$, where $D$ is the diffusion coefficient, as long as $t_{d}<\tau_{\mathrm{sf}}$, the spin-flip time, or, equivalently, $L<\sqrt{D \tau_{\mathrm{sf}}}$, the spin-diffusion length. These other moments start to move by the torque they experience by absorbing part of the diffusing spin accumulation, and, in turn, emit spin currents by themselves. When magnetic dynamics are slow on the scale of the diffusion time $t_{d}$, the retardation of spin diffusion may be disregarded and the dynamic exchange coupling is practically instantaneous. This is the regime we treat in the present section. (A similar discussion applies to the ballistic transport regime.) The dynamic coupling between moving magnetizations by the exchange of nonequilibrium spin currents is affected by spin-conserving random scattering much less than the rapid suppression of the static coupling.

When the normal spacer is much thinner than the spin-diffusion length, the dynamic spin exchange is governed by $\mathcal{A}_{F|N| F}^{\uparrow}$, Eq. (82), which does not depend on the $N$-spacer width $L$ for ballistic spacers. The coupling decays as $1 / L$ for spacer widths larger than the scattering mean free path and is exponentially suppressed when $L$ becomes larger than the spin-diffusion length. The dynamic exchange interaction is less sensitive to disorder than its static counterpart because the latter relies on the orbital quantum interference between electron trajectories connecting magnetic moments or layers, whereas the former requires spin coherence on traversing the normal spacer. Dynamic exchange coupling was first addressed perturbatively in the context of electron spin resonance by Barnes (1974), who pointed out its longrange nature as compared to static coupling. However, dynamic correlations between an individual magnetic moment and proximate conduction electrons (which, as described in Sec. IV, lead to magnetization relaxation) were overlooked at that time. In the context of ferromagnetic-resonance experiments, dynamic exchange coupling has been studied by Hurdequint and
Malouche (1991) and more recently by Heinrich, Tserkovnyak, et al. (2003) and Lenz et al. (2004).

The spin-pumping picture of dynamic exchange coupling is confirmed by recent experiments with sufficiently large normal spacers (Heinrich, Tserkovnyak, et al., 2003), as will be discussed in detail below. In the limit when static coupling becomes appreciable, the spin-pumping-based circuit analysis leading to Eq. (82) may not hold exactly, however, since the circuit model of Sec. II assumes that the interfaces and normal spacers scramble the electron distribution in momentum space. A sizable static exchange is thus in principle inconsistent with the key assumption underlying the circuit model. A rigorous treatment of dynamic coupling in the regime of significant static exchange coupling is difficult, as explained in Sec. VII.A.2. Here we heuristically assume that dynamic coupling as described by spin-pumping theory still holds in the presence of a residual static exchange interaction, which turns out to be sufficient to make a connection with some recent experiments (Lenz et al., 2004). This picture can be justified by the general observation that in intermetallic heterostructures quantum-size effects often modulate only weakly the semiclassical transport contribution.

In the following, we consider the collective dynamics of a $F|N| F$ structure, i.e., two magnetic films separated by a normal metal. Possible static exchange through very thin spacers is taken into account phenomenologically by postulating a Heisenberg-type contribution (per cross-sectional unit area)

$$
E_{x}=-J \mathbf{m}_{1} \cdot \mathbf{m}_{2}
$$

to the magnetic free-energy functional that determines effective fields (6) experienced by the ferromagnets. $J$ is the Heisenberg coupling constant, which is assumed to be small compared to the magnetic bulk exchange stiffness $A$ divided by the magnetic-film thickness $d$, $|J| \ll A / d$. This assumption is necessary in order to treat each individual magnetic layer as a macrospin pointing along a unit vector $\mathbf{m}_{i}$. $J$ depends in an oscillatory fashion on the spacer-layer thickness and favors either parallel $(J>0)$ or antiparallel $(J<0)$ orientation of the magnetic layers, as discussed in Sec. I.B. In nanostructured pillars, the magnetostatic interaction can also favor an antiparallel coupling of the form (84), even at spacer layer thicknesses at which the exchange coupling vanishes.

We consider magnetic films that are thinner than $A /|J|$ but thicker than $\lambda_{\mathrm{sc}}$ [Eq. (13)], so that they completely absorb transverse spin currents. (Note that in typical metallic structures, $A /|J| \gg \lambda_{F}$ and $\lambda_{\mathrm{sc}} \sim \lambda_{F}$.) A precessing magnetization vector $\mathbf{m}_{i}$ of ferromagnet $F_{i}$ pumps spin angular momentum at the rate (38) determined by the spin-pumping efficiency $\mathcal{A}_{F|N| F}^{\uparrow}$ [Eq. (82)] into the normal-metal spacer. We concentrate here on smallangle excitations of a collinear magnetic equilibrium configuration. Equation (82) applies to the typical situation in which magnetization dynamics are slow on the characteristic time scales for electron transfer across the 


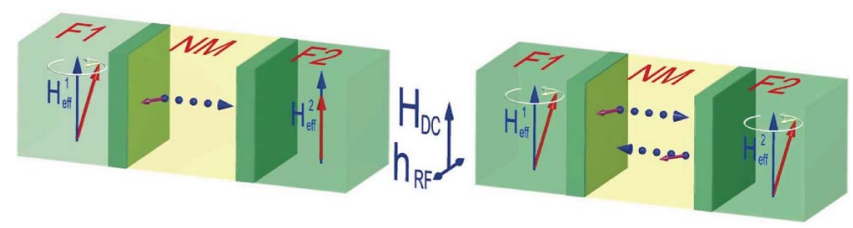

FIG. 11. (Color online) A cartoon of the dynamic-coupling phenomenon. In the left drawing, the layer $F_{1}$ is at resonance and its precessing magnetic moment pumps spin current into the spacer, while $F_{2}$ is detuned from its FMR. In the right drawing, both films resonate at the same external field, inducing spin currents in opposite directions. The short arrows in $N$ indicate the instantaneous direction of the spin angular momentum $\propto \mathbf{m}_{i} \times \dot{\mathbf{m}}_{i}$ carried away by the spin currents. Darker areas in $F_{i}$ around the interfaces represent the narrow regions in which the transverse spin momentum is absorbed. From Heinrich, Tserkovnyak, et al., 2003.

spacer. When one ferromagnet is stationary, see the left drawing in Fig. 11, the dynamics of the other film are governed by the Landau-Lifshitz-Gilbert equation (8) but with the effective damping parameter enhanced with respect to the intrinsic value, as given by Eq. (83). When both magnetizations are allowed to move, see the right sketch in Fig. 11, the coupled Landau-Lifshitz-Gilbert equations expanded to take into account the spin torques (14) read

$$
\begin{aligned}
\frac{d \mathbf{m}_{i}}{d t}= & -\gamma_{i} \mathbf{m}_{i} \times\left(\mathbf{H}_{\mathrm{eff}, i}+\frac{J \mathbf{m}_{j}}{M_{s, i} d_{i}}\right)+\alpha_{i} \mathbf{m}_{i} \times \frac{d \mathbf{m}_{i}}{d t} \\
& +\alpha_{i}^{\prime}\left(\mathbf{m}_{i} \times \frac{d \mathbf{m}_{i}}{d t}-\mathbf{m}_{j} \times \frac{d \mathbf{m}_{j}}{d t}\right),
\end{aligned}
$$

where $\mathbf{H}_{\text {eff }, i}$ are effective fields not including the exchange contribution (84), $\alpha_{i}^{\prime}=\hbar \gamma_{i} \operatorname{Re} \mathcal{A}_{F|N| F}^{\uparrow} / 4 \pi M_{s, i} d_{i} S$ is given by Eq. (83) having disregarded $\operatorname{Im} \mathcal{A}_{F|N| F}^{\uparrow \downarrow}$, and $j$ $=1(2)$ for $i=2(1)$. As a first simple example, consider the parallel equilibrium configuration, $\mathbf{m}_{1}^{(0)}=\mathbf{m}_{2}^{(0)}$, with zero static coupling, $J=0$, and matched resonance conditions: $\alpha_{1}=\alpha_{2}=\alpha$ and $\gamma_{1} \mathbf{H}_{\mathrm{eff}, 1}=\gamma_{2} \mathbf{H}_{\mathrm{eff}, 2}$, with magnetizationindependent effective fields $\mathbf{H}_{\mathrm{eff}, i}$. After linearizing Eq. (85) in terms of small deviations $\mathbf{u}_{i}(t)=\mathbf{m}_{i}(t)-\mathbf{m}_{i}^{(0)}$ of the magnetization direction $\mathbf{m}_{i}$ from its equilibrium value $\mathbf{m}_{i}^{(0)}$, we immediately see that $\mathbf{u}=\left(\mathbf{u}_{1} s_{1}+\mathbf{u}_{2} s_{2}\right) /\left(s_{1}+s_{2}\right)$, where $s_{i}=\gamma_{i} M_{s, i} d_{i}$, viz., the symmetric mode, is damped with the intrinsic Gilbert parameter $\alpha$, whereas the difference $\Delta \mathbf{u}=\mathbf{u}_{1}-\mathbf{u}_{2}$, viz., the antisymmetric mode, relaxes with enhanced damping constant $\alpha=\alpha+\alpha_{1}^{\prime}+\alpha_{2}^{\prime}$. This demonstrates that the dynamic interaction can lead to nontrivial collective magnetization dynamics even when the static interaction vanishes.

Let us now analyze a more general case of a coupled magnetic bilayer undergoing a collective circular precession near a parallel equilibrium configuration $\mathbf{m}_{1}^{(0)}$ $=\mathbf{m}_{2}^{(0)}=\hat{\mathbf{z}}$. For simplicity, we still focus on a nearly symmetric structure by setting $\gamma_{i}=\gamma, \gamma J / M_{s, i} d_{i}=\omega_{x}, \alpha_{i}=\alpha$, and $\alpha_{i}^{\prime}=\alpha^{\prime}$, but allow the effective fields $\gamma \mathbf{H}_{\text {eff }, i}=\omega_{i} \hat{\mathbf{z}}$ to differ, $\omega_{1} \neq \omega_{2}$. Equation (85) then reduces to

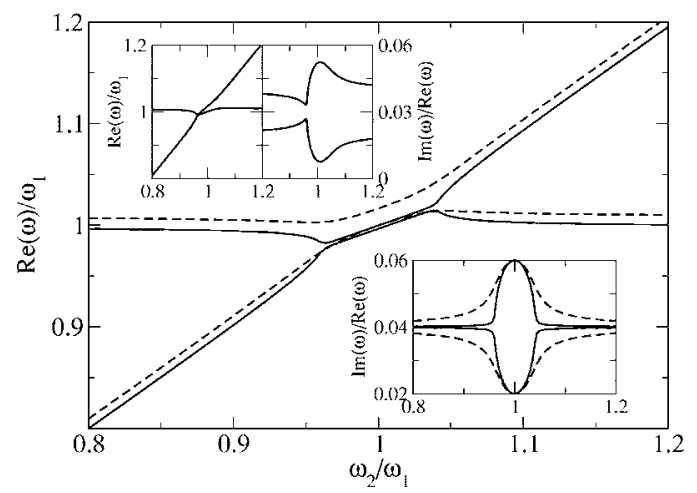

FIG. 12. The main panel displays the real part of the two frequencies $\omega$ which solve Eq. (86) for linearized dynamics of a symmetric magnetic bilayer close to the parallel magnetization configuration. Solid lines are obtained by setting the static coupling to zero, $\omega_{x}=0$, and dashed lines for $\omega_{x}=0.01 \omega_{1}$. The lower inset shows the corresponding imaginary part of $\omega$. When $\omega_{x}=0$ and near the frequency crossing, $\omega_{1} \approx \omega_{2}$, the symmetric mode has a smaller imaginary part (less damping) and only slightly larger real part. For $\omega_{x}<0$, the symmetric mode has a larger (real part of the) frequency than the antisymmetric mode, whereas the antisymmetric mode acquires a higher frequency for sufficiently large and positive $\omega_{x}$. In all cases, the antisymmetric mode remains more strongly damped. The upper inset shows the results for $\omega_{x}=0.01 \omega_{1}$ but setting $\alpha_{1}=0$ while keeping a finite $\alpha_{2}$. This inset illustrates the effect of a bilayer asymmetry.

$$
\begin{aligned}
\frac{d \mathbf{m}_{i}}{d t}= & \omega_{i} \hat{\mathbf{z}} \times \mathbf{m}_{i}+\omega_{x} \mathbf{m}_{i} \times \mathbf{m}_{j}+\alpha \mathbf{m}_{i} \times \frac{d \mathbf{m}_{i}}{d t} \\
& +\alpha^{\prime}\left(\mathbf{m}_{i} \times \frac{d \mathbf{m}_{i}}{d t}-\mathbf{m}_{j} \times \frac{d \mathbf{m}_{j}}{d t}\right) .
\end{aligned}
$$

The linearized equations of motion in the absence of driving force are solved by the form $\propto \exp (i \omega t)$ with two complex-valued natural frequencies $\omega$ and definite circular polarization. The solid lines in the main panel of Fig. 12 show the real part of these $\omega$ 's for various ratios $\omega_{2} / \omega_{1}$ after setting $\omega_{x}=0$, and the dashed lines after setting $\omega_{x} / \omega_{1}=0.01$, i.e., introducing a finite static exchange coupling. In both cases, $\alpha=\alpha^{\prime}=0.02$. The lower inset shows the corresponding normalized imaginary part of $\omega$. For $\omega_{x}=0$ and resonance frequencies $\omega_{1}$ and $\omega_{2}$ well separated on the scale of the enhanced damping $\alpha^{\prime}$, viz., $\left|\omega_{2} / \omega_{1}-1\right| \gg 2 \alpha^{\prime}$ (assuming $\alpha^{\prime} \ll 1$ ), the dynamics of two ferromagnets decouple and the spin pumping can be accounted for by simply adding $\alpha^{\prime}$ to the effective Gilbert parameter of each $F$ layer. When, on the other hand, $\left|\omega_{2} / \omega_{1}-1\right| \lesssim 2 \alpha^{\prime}$, the spin pumping locks the collective dynamics to independent symmetric (acoustic) and antisymmetric (optic) normal modes with frequencies that are nearly degenerate and close to $\left(\omega_{1}+\omega_{2}\right) / 2$. It then follows from Eq. (86) that the symmetric mode is weakly damped, with $\operatorname{Im}(\omega) / \operatorname{Re}(\omega)$ close to $\alpha$, whereas the antisymmetric mode experiences an enhanced Gilbert damping $\alpha+2 \alpha^{\prime}$. 


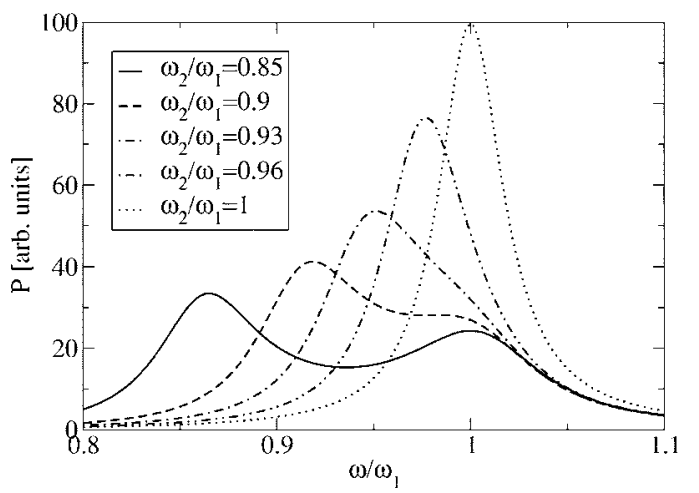

FIG. 13. Dissipation power $P$ of a symmetric bilayer in the presence of a uniform circularly polarized rf driving field at frequency $\omega$. The parameters are the same as those used to generate the dashed lines in Fig. 12.

The presence of a static exchange interaction lifts the frequency of the antisymmetric mode by $2 \omega_{x}$, not affecting the symmetric mode. When the frequencies $\omega_{i}$ are well separated, the static interaction lifts both of them by $\omega_{x}$. One of the modes then acquires some symmetric and the other some antisymmetric character even when $\left|\omega_{2} / \omega_{1}-1\right| \geqslant 2 \alpha^{\prime}$, as illustrated by the damping of both modes (dashed lines in the lower inset of Fig. 12). In the upper inset of Fig. 12, the calculations are repeated for finite $\omega_{x}$, but setting the intrinsic damping of one of the films to zero, $\alpha_{1}=0$, while $\alpha_{2}=\alpha_{i}^{\prime}=0.02$. The dynamic locking into symmetric or antisymmetric modes close to the resonance crossing has disappeared. The modes are harder to synchronize when the participating modes have different amplitudes. A significant locking may be expected only in a symmetric bilayer with the individual films having similar resonant modes near parallel equilibrium axes. In particular, a bilayer in an antiparallel equilibrium configuration is not disposed to dynamic locking, since individual layers have excitations with opposite circular (or elliptic, in the presence of anisotropies) polarizations.

We now turn to a discussion of the consequences for the observable in FMR experiments, viz., the energydissipation power $P$ of dynamically locked collective dynamics as plotted in Fig. 13 as a function of the frequency $\omega$ of the uniform and circularly polarized transverse rf driving field. The system parameters are the same as those used to generate the dashed lines in Fig. 12. For the smallest ratio $\omega_{2} / \omega_{1}$ in Fig. 13, the two bilayer excitations are recognized as separated Lorentzian peaks with half-widths close to $\left(\alpha+\alpha^{\prime}\right) \omega$; see Eq. (11). When the elementary bilayer excitations become locked at $\left|\omega_{2} / \omega_{1}-1\right| \lesssim 2 \alpha^{\prime}$, the rf radiation excites only the symmetric mode, and the two Lorentzians merge into a single sharper Lorentzian with half-width of only $\alpha \omega$. This narrowing is explained by the cancellation of spin currents and can be quite dramatic when $\alpha \ll \alpha^{\prime}$.

In FMR experiments, the applied magnetic field is swept, whereas the rf frequency $\omega$ is fixed by a resonant cavity. In Sec. IV.C, we discussed FMR experiments by

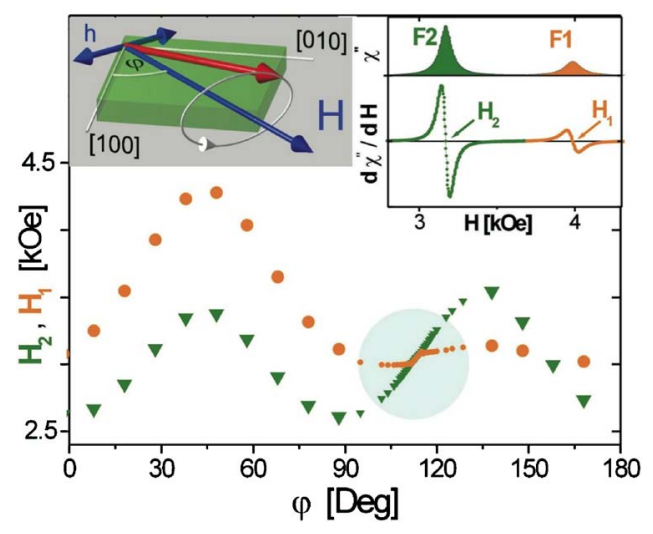

FIG. 14. (Color online) Dependence of the static FMR fields $H_{1}$ (circles) and $H_{2}$ (triangles) of the thin Fe film $\left(F_{1}\right)$ and the thick Fe film $\left(F_{2}\right)$, respectively, on the angle $\varphi$ of the static magnetic field with respect to the Fe [100] crystallographic axis. The sketch of the in-plane measurement in the left inset shows how the rf magnetic field (double-pointed arrow) drives the magnetization (on a scale grossly exaggerated for easy viewing). The right inset shows the measured absorption peaks for layers $F_{1}$ and $F_{2}$ at $\varphi=60^{\circ}$. The absorption power is given by the imaginary part of the susceptibility of the rf magnetization component along the rf driving field, which is denoted by $\chi^{\prime \prime}$. From Heinrich, Tserkovnyak, et al., 2003.

Urban et al. (2001) on magnetic bilayers in which one of the layers remains close to equilibrium while the other is resonantly excited. The collective magnetization dynamics were measured by Heinrich, Tserkovnyak, et al. (2003) in the same system making use of an accidental crossing of the resonance frequencies when the static field is reoriented relative to the crystal anisotropy axes. The resulting spectra could be quantitatively explained in terms of the dynamic coupling in the limit of a vanishing static exchange interaction. We summarize their findings in the following.

The molecular-beam-epitaxy-grown structure of Heinrich, Tserkovnyak, et al. (2003) incorporates two ferromagnetic films, a thinner 16-monolayer $\left(F_{1}\right)$ and a thicker 40-monolayer $\left(F_{2}\right)$ Fe film, separated by 40 monolayers of $\mathrm{Au}$, grown on $\mathrm{GaAs}$ and capped with $\mathrm{Au}$, i.e., the stacking order is $\mathrm{GaAs}|16 \mathrm{Fe}| 40 \mathrm{Au}|40 \mathrm{Fe}| 20 \mathrm{Au}(001)$. The uniaxial magnetic anisotropy in $F_{1}$ at the GaAs|Fe interface can be used to intentionally tune the resonance fields for $F_{1}$ and $F_{2}$ into a crossover by rotating the static magnetic field by an angle $\varphi$ with respect to the (001) crystal axis. In a finite interval of $\varphi$ near the crossover (shaded area in Fig. 14), the two FMR fields clearly "stick" to each other, a phenomenon explained above. When the resonance fields are identical, $H_{1}=H_{2}$, the rf magnetization components of $F_{1}$ and $F_{2}$ are moving in phase as depicted in the right drawing in Fig. 11. For similar trajectories of $F_{1}$ and $F_{2}$, the total spin current through the spacer vanishes resulting in zero excess damping for both films, as follows from Eq. (85). The locked collective motion is then hindered only by the intrinsic local damping. This is experimentally verified, as shown in 

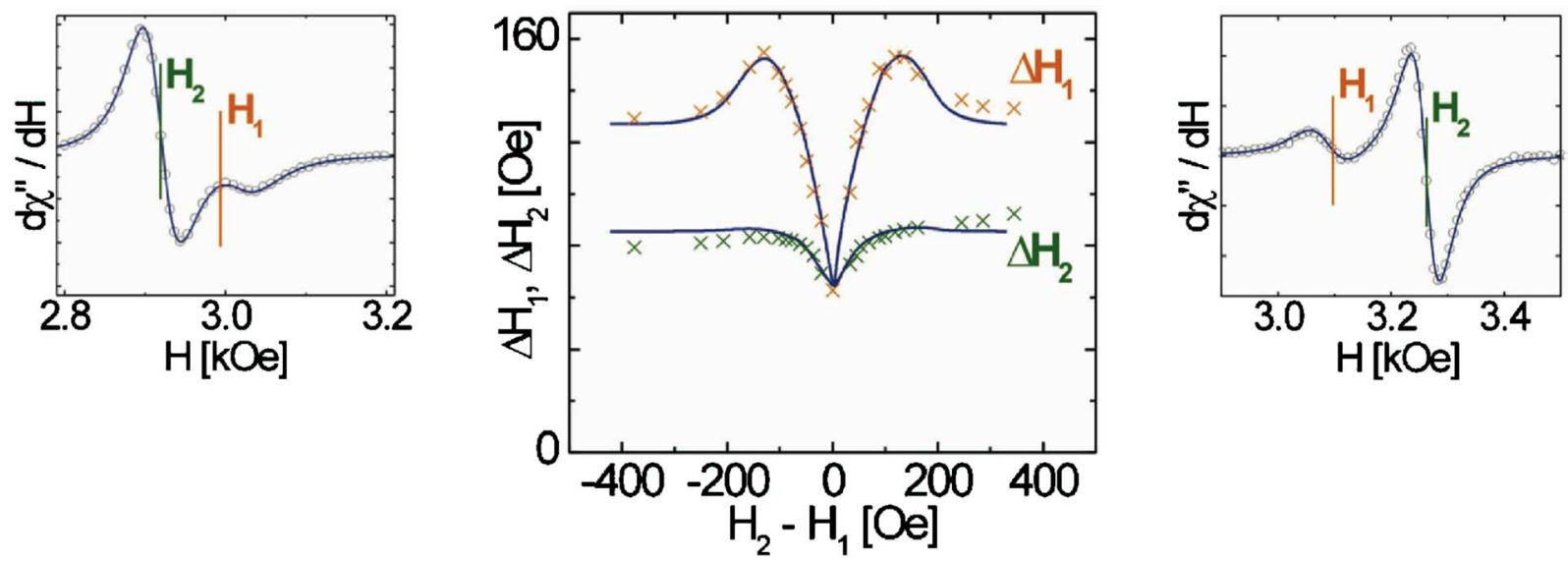

FIG. 15. (Color online) Comparison of theory (solid lines) with room-temperature measurements (symbols) close to and at the crossover of the FMR fields, marked by the shaded area in Fig. 14. The left and right frames show FMR signals for the field difference, $H_{2}-H_{1}$, of -78 and +161 Oe, respectively. The theoretical results are parametrized with the full set of magnetic parameters which were measured independently (Urban et al., 2001). The magnitude of the spin-pump current was determined by the linewidth at large separation of the FMR peaks and agrees well with that predicted theoretically; see Sec. IV.C. The middle frame displays the effective FMR linewidth of magnetic layers for the signals fitted by two Lorentzians as a function of the external field. At $H_{1}=H_{2}$, the FMR linewidths reached their minimum values at the level of intrinsic Gilbert damping of isolated films. The calculations in the middle frame did not take small variations of the intrinsic damping with angle $\varphi$ into account, which resulted in deviations between theory and experiment for larger $\left|H_{1}-H_{2}\right|$. Notice that $\Delta H_{1}$ first increases before attaining its minimum, which is due to a contribution of the antisymmetric collective mode. As a side comment, it should be noted that although fitting the absorption signal by two Lorentzians is a legitimate approach to comparing theoretical calculation with experimental curves, the analysis does not imply that the signal is always well approximated by the sum of two Lorentzians, which may not be the case very close to the FMR-field crossover. From Heinrich, Tserkovnyak, et al., 2003.

Fig. 15. For a theoretical analysis, Heinrich, Tserkovnyak, et al. (2003) solved Eq. (85), taking into account the ellipticity of the magnetic motion caused by the anisotropies. Using parameters derived from measurements on the uncoupled layers, they calculated the total FMR signal as a function of the difference between the resonance fields, $\mathrm{H}_{2}-\mathrm{H}_{1}$, without additional fitting parameters. The predictions are compared with the measurements in Fig. 15. The remarkably good agreement between experiment and theory provides strong evidence that the dynamic exchange coupling not only contributes to the damping but leads to a new collective behavior of magnetic heterostructures. Heinrich, Tserkovnyak, et al. (2003) additionally carried out measurements on samples with Au spacer thicknesses between 14 and 100 monolayers. The reported weak dependence of the FMR response on the spacer thickness proves the long range of the dynamic interaction.

Lenz et al. (2004) have investigated collective FMR dynamics in $\mathrm{Ni}|\mathrm{Cu}| \mathrm{Ni}$ and $\mathrm{Ni}|\mathrm{Cu}| \mathrm{Co}$ structures with $\mathrm{Cu}$ thicknesses down to 2 monolayers. Such thin $\mathrm{Cu}$ spacers support a sizable static coupling between magnetic films. Like Heinrich, Tserkovnyak, et al. (2003), Lenz et al. (2004) have observed a sharp drop in the linewidth near an FMR-field crossing. Far from it, the optic-mode resonance is systematically broader than that of the acoustic mode, consistent with the spin-pumping mechanism. The difference between the optic and acoustic linewidths exhibits an oscillatory dependence on the $\mathrm{Cu}$ spacer thickness, which roughly follows the predicted exchange-coupling constant $J$. As shown in the lower inset of Fig. 12, a stronger coupling $J$ would indeed result in a larger asymmetry in the two linewidths, away from the crossing, which could lead to oscillations in the linewidth difference. However, an inhomogeneous spread of the coupling strengths may also contribute to the broadening which depends nonmonotonically on the spacer thickness, providing an alternative explanation for the linewidth oscillations.

In the regime of very strong static exchange coupling, $|J| \geq A / d$, opposite to what was assumed so far, magnetization gradients across the bulk magnetic layers alleviate the energy cost of the discontinuity in the magnetic orientation between two magnetic layers. This reduces the spin pumping through the spacer, and thus the additional broadening of the antisymmetric mode because the adjacent magnetizations are better locked by the strong static exchange interaction.

\section{B. Magnetic superlattices}

Magnetic multilayers display a rich pattern of physical properties that have been well investigated [see, e.g., Camley and Stamps (1993) for a review]. However, the relevance of dynamic exchange coupling discussed in the previous subsection on the spin-wave dispersions and lifetimes appears to have not been recognized yet. In the following, we present a simple model description that should suffice to estimate the order of magnitude of the predicted effects that we hope will stimulate new experiments. We also remark that superlattices can serve a 


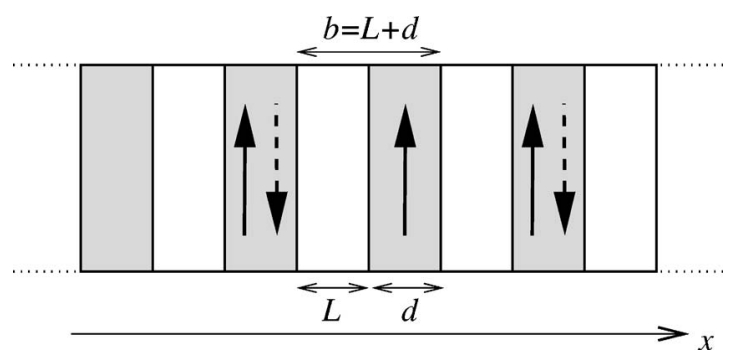

FIG. 16. A schematic view of the superlattice and its geometric parameters as considered in the text. A $F \mid N$ bilayer is repeated along the $x$ axis, with either ferromagnetic or antiferromagnetic alignment of the consecutive magnetic layers. The system is translationally invariant along the two remaining axes.

theoretical purpose as toy models for describing certain features of bulk magnetism.

Consider a periodic stack (in the $x$ direction) of alternating $F$ and $N$ layers forming a two-component superlattice. We consider the model depicted in Fig. 16 in which a $F \mid N$ bilayer forms the unit cell with thickness $b=L+d$, and in which the normal-metal spacer of width $L$ separates the magnetic films of thickness $d \gg \lambda_{\mathrm{sc}}$. Translational invariance is assumed in the lateral directions. We consider here collective spin-wave excitations, taking both static and dynamic exchange couplings into account in exactly the same fashion as in the previous subsection on magnetic bilayers, modeling each magnetic layer as a single macrospin.

Small-angle magnetization dynamics of a multilayer in an all-parallel configuration are described in terms of local deviations from the equilibrium: $\mathbf{u}_{i}(t)=\mathbf{m}_{i}(t)-\mathbf{m}^{(0)}$. For long-wavelength excitations, it may be approximated as a continuous function $\mathbf{u}(x, t)$ of the coordinate $x$ normal to the interfaces. For a uniaxial effective field $H_{\text {eff }}=H_{\text {eff }} \mathbf{m}^{(0)}$, the spin-wave dynamics obey the differential equation

$\partial_{t} \mathbf{u}=\mathbf{m}^{(0)} \times\left[\omega_{0} \mathbf{u}-\omega_{x} b^{2} \partial_{x x} \mathbf{u}+\alpha \partial_{t} \mathbf{u}-\alpha^{\prime} b^{2} \partial_{x x, t} \mathbf{u}\right]$,

where we have used the quantities defined for magnetic bilayers in Sec. V.A: $\omega_{x}=\gamma J / M_{s} d, \quad \alpha^{\prime}=\hbar \gamma \operatorname{Re} A_{F|N| F}^{\uparrow \dagger} /$ $4 \pi M_{s} d S$, and $\omega_{0}=\gamma H_{\text {eff }}$. The second term on the righthand side of Eq. (87) is due to static exchange interaction mediated by quantum-well states of the spacer layers, and in the last term we recognize the dynamic coupling induced by the spin pumping. The second spatial derivatives simply reflect the difference of the spin currents through two consecutive normal spacers in the continuum limit. The static Heisenberg coupling can be interpreted as the superlattice equivalent of the bulk exchange stiffness parameter $A$, which for the superlattice is given by $\tilde{A}=J b^{2} / d$. Both $\omega_{x}$ and $\alpha^{\prime}$ depend on the normal-interlayer thickness $L$. It follows from Eq. (87) that the small-momentum, $k \ll b^{-1}$, spin-wave excitations of the superlattice, propagating perpendicular to the interfaces, $\propto \exp \{i[k x-\omega(k) t]\}$, obey a dispersion relation

$$
\omega(k)=\frac{\omega_{0}+(b k)^{2} \omega_{x}}{1+i\left[\alpha+(b k)^{2} \alpha^{\prime}\right]} .
$$

When $k \rightarrow 0, \omega(k)$ reduces to the Larmor frequency $\omega_{0}$ of the individual magnetic layers because static and dynamic exchange couplings vanish when the consecutive magnetic layers move coherently in phase, as explained in Sec. V.A. Equation (88) holds for momenta comparable to $b^{-1}$ when $b k$ is replaced by $2 \sin (b k / 2)$.

The situation is very different for an antiferromagnetically aligned superlattice, which is the lowest-energy state when, for example, $J<0$ and $H_{\text {eff }}=0$. In this case,

$$
\omega(k)=\frac{-i \omega_{x}\left[2 \alpha+(b k)^{2} \alpha^{\prime} \pm \sqrt{4 \alpha^{2}-(b k)^{2}\left(1+\alpha^{2}\right)}\right]}{1+\alpha^{2}+4 \alpha \alpha^{\prime}+\alpha^{\prime}(b k)^{2}},
$$

where plus and minus signs refer, respectively, to the modes with antisymmetric and symmetric dynamics in adjacent layers for overdamped motion, and to the rightand left-propagating modes when the real part of $\omega(k)$ is significant. Note that now $\omega_{x}<0$, so that $\operatorname{Im} \omega>0$, as required for a stable configuration. In the absence of bulk magnetization damping, $\alpha=0$, Eq. (89) reduces to

$$
\omega(k)=\frac{ \pm(b k) \omega_{x}}{1 \pm i(b k) \alpha^{\prime}},
$$

with linear dispersion and damping at small $k$. Equations (89) and (90) can also be generalized to large momenta by replacing $b k$ with $2 \sin (b k / 2)$. Notice that in Eqs. (87), (88), and (90), dynamic coupling modifies the damping similarly to the way static coupling affects the excitation frequency of the magnetic superlattice. Crystal and shape anisotropies on top of the simple effective fields assumed above might become important in real structures, and their inclusion is straightforward.

FMR experiments access the multilayer dynamics from the sample surface down to the microwave skin depth $\lambda_{\text {skin }} \sim 100 \mathrm{~nm}$ (which is even smaller in Brillouin light scattering). Therefore only modes with momenta $k \gtrsim \pi / \lambda_{\text {skin }}$ can be measured. Since the skin depth decreases when the temperature is lowered, both the FMR frequency and the damping in a ferromagnetically aligned multilayer should grow roughly as $1 / \lambda_{\text {skin }}^{2} \propto \tau$, the momentum scattering time in the normal skin-effect regime. Such studies can thus give information about the temperature-dependent scattering in superlattices; a weak temperature dependence could indicate that scattering is dominated by structural disorder. Inelastic neutron-scattering spectroscopy may be useful in elucidating the collective dynamics in thick multilayers, especially if supported by elastic neutron scattering (Fitzsimmons et al., 2004) which probes the magnetic profile in the superlattice.

\section{Large-angle motion in biased spin valves}

Perpendicular spin valves, i.e., $F_{s}|N| F_{h}$ trilayer pillar structures with layer thicknesses down to a few mono- 
layers and submicron lateral dimensions, are ideal systems for studying precession and switching phenomena in magnetic heterostructures. By attaching contacts on the outer sides an electric bias can be applied perpendicular to the interface planes. In many experiments, $F_{s}$ is a "soft" ferromagnetic film with a magnetization that can change easily, whereas $F_{h}$ is a "hard" magnetic layer whose magnetization is assumed to be stationary. The relevant variable is then the time-dependent magnetization of the soft layer. The soft layer can be excited by a current bias or an applied rf magnetic field (or both). In the second case (realized as, e.g., an isolated magnetic bilayer of Secs. IV.C and V.A), $F_{h}$ can be pinned by an exchange bias or surface magnetic anisotropy (Urban et al., 2001). In the former case, the magnetization of layer $F_{h}$ may also be rendered less sensitive to a given spin torque simply by growing it much thicker than $F_{s}$ or by a resistance anisotropy (Kovalev et al., 2002). For a sufficiently thick spacer $N$, the static interaction between the ferromagnetic layers can be disregarded, while the dynamic coupling induced by the spin pumping may still be sizable (see Sec. V.A).

Berger (1996) and Slonczewski (1996) predicted that spin valves should display time-dependent effects. Slonczewski (1996) realized that a current flowing through a spin valve causes a spin transfer through the nonmagnetic spacer, inducing spin torques on the ferromagnets. Berger (1996) predicted that two ferromagnets should interact even without an applied electric current, resulting in a significant contribution to the Gilbert damping of magnetization dynamics. He further showed that an electric current can excite zero-momentum spin waves in the ferromagnet, an idea that was later supported experimentally; see, e.g., Tsoi et al. (2000). The condition for the resonant spin-wave emission (Berger, 1996) is similar to the criterion for magnetization reversal by Slonczewski (1996, 1999), who treated the Gilbert damping parameter as a phenomenological constant. Berger (2001), however, found a dependence of the damping parameter in spin valves on the relative magnetization angle. Some of Berger's and Slonczewski's results, as well as the underlying theoretical models, are thus not consistent with each other. The spin-pumping concept unified the seminal work of these pioneers (Tserkovnyak et al., 2003b) as explained in the remainder of this subsection. We shall calculate the critical current bias for the low-temperature magnetization instability and the configuration-dependent Gilbert damping parameter. The treatment here is limited to the macrospin model, i.e., to small systems in which magnetic layers are monodomain ferromagnets characterized by two magnetization vectors. However, if combined with micromagnetic simulations (Lee et al., 2004), in principle the full range of the precession and switching dynamics can be studied.

Consider the system sketched in Fig. 17. The $F_{s}|N| F_{h}$ trilayer is sandwiched between two normal-metal contacts sustaining a charge-current bias $I_{c}$. The soft layer $F_{s}$ magnetization $\mathbf{m}_{1}$ then starts moving from its equilibrium direction at a critical value that depends on the

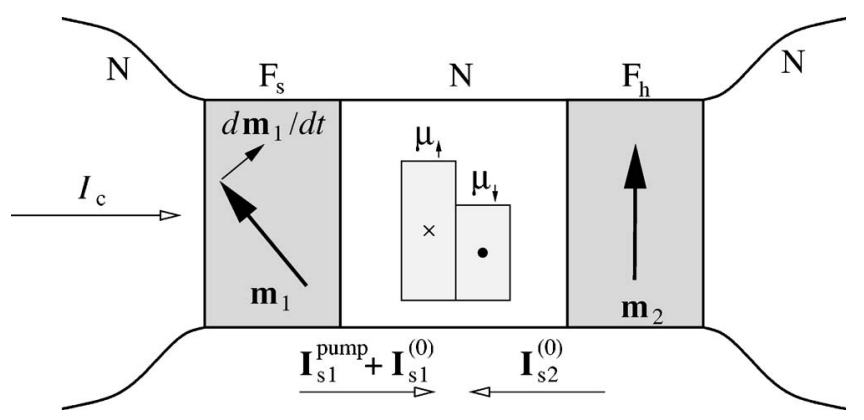

FIG. 17. Schematic of a current-biased spin valve (a "dynamic" version of Fig. 1). The symbols are explained in the text.

applied magnetic field. Thermal activation facilitates current-induced magnetization switching (Myers et al., 2002), but we focus here on the low-temperature regime. The spin torque on the magnetization of $F_{s}$ in the presence of a spin current $\mathbf{I}_{s 1}$ flowing from $F_{s}$ into the normal spacer is given by Eq. (14). The spin current

$$
\mathbf{I}_{s 1}=\mathbf{I}_{s 1}^{\text {exch }}+\mathbf{I}_{s 1}^{\text {bias }}
$$

consists of the dynamic exchange current $\mathbf{I}_{s 1}^{\text {exch }}$ induced by spin pumping (38) and of the current $\mathbf{I}_{s 1}^{\text {bias }}$ driven by an applied current bias. The former is responsible for a dynamic coupling between the ferromagnets, see Sec. V.A, and, as we discuss in the following, can be interpreted as a viscous friction term that stabilizes the relative magnetization configuration of the spin valve against the torques exerted by $\mathbf{I}_{s}^{\text {bias }}$ or an applied magnetic field. In high-density metallic systems, the applied voltages and spin accumulations are safely smaller than the Fermi energies, which means that we are in the linear-response regime and both spin currents may be calculated independently of each other. Spin pumping in the outward direction, i.e., into the external connectors, would only increase the intrinsic damping coefficient by a constant value, as discussed in Secs. IV.A and IV.B, and can thus be added trivially to the intrinsic damping $\alpha$. Spin pumping into the normal spacer, which gives rise to $\mathbf{I}_{s 1}^{\text {exch }}$, requires more attention.

We start by considering the spin current (38) pumped into the spacer by a time-dependent $\mathbf{m}_{1}(t)$ in the absence of an applied current bias, $I_{c}=0$. The following assumptions are convenient and realistic: (i) the magnetic films are sufficiently thicker than $\lambda_{\mathrm{sc}}$ so that the transmission contribution to the spin-pumping parameter $\mathcal{A}^{\uparrow \downarrow}$ can be disregarded, (ii) the mixing conductance $\tilde{g}^{\uparrow \downarrow}$ is realvalued, (iii) two magnetic films including the interfaces have the same conductances, (iv) the normal spacer is ballistic, and (v) spin-flip processes are restricted to the ferromagnetic layers with the spin-diffusion length $\lambda_{\text {sd }}$ $\gg \lambda_{\text {sc. }}$. Note that in this subsection $\lambda_{\text {sd }}$ denotes the spindiffusion length inside the ferromagnets for longitudinal spin transport along the magnetization direction.) The spin currents out of the magnetic layers into the normal spacer are then given by $\mathbf{I}_{s i}=\mathbf{I}_{s i}^{\text {pump }}+\mathbf{I}_{s i}^{(0)}$ with 


$$
\begin{aligned}
\mathbf{I}_{s i}^{\text {pump }} & =\frac{\hbar}{4 \pi} \tilde{g}_{r}^{\uparrow \downarrow} \mathbf{m}_{i} \times \frac{d \mathbf{m}_{i}}{d t}, \\
\mathbf{I}_{s i}^{(0)}= & -\frac{1}{4 \pi}\left(\frac{2 \tilde{g}^{\uparrow \uparrow} \tilde{g}^{\downarrow \downarrow}}{\tilde{g}^{\uparrow \uparrow}+\tilde{g}^{\downarrow \downarrow}} \mathbf{m}_{i}\left(\Delta \boldsymbol{\mu}_{s i} \cdot \mathbf{m}_{i}\right)\right. \\
& \left.+\tilde{g}_{r}^{\uparrow \downarrow} \mathbf{m}_{i} \times \Delta \boldsymbol{\mu}_{s i} \times \mathbf{m}_{i}\right),
\end{aligned}
$$

where the last equation is derived using Eqs. (25) and (26) and a circuit-theory analysis discussed in Sec. II.A, assuming vanishing charge current. Here $\mathbf{m}_{i}$ is the $i$ th-layer magnetization direction and $\Delta \boldsymbol{\mu}_{s i}=\boldsymbol{\mu}_{s N}-\mu_{s F i} \mathbf{m}_{i}$ is the spin-accumulation difference across the $N \mid F_{i}$ interface. The magnetization-precession period is typically much longer than the electron dwell time in metallic spacers. Assuming weak spin-flip scattering in $N$, conservation of angular momentum then implies that $\mathbf{I}_{s 1}+\mathbf{I}_{s 2}$ $=\mathbf{0}$. It is now straightforward to calculate the exchange spin current which is given by $\mathbf{I}_{s 1}^{\text {exch }}=\mathbf{I}_{s 1}$ when $I_{c}=0$.

The longitudinal component of the spin accumulation $\boldsymbol{\mu}_{s N}$ can penetrate into ferromagnets on the scale of the spin-diffusion length $\lambda_{\text {sd }}$, whereas the transverse component vanishes on the shorter scale of $\lambda_{\mathrm{sc}}$ near the interface. The longitudinal spin accumulation in the ferromagnet $\mu_{s F i}$, and thereby $\mathbf{I}_{s i}^{(0)}$, can be obtained for a given $\mu_{s N}$ from the diffusion equation for the (longitudinal) spin transport in the ferromagnets, similarly to the normal-layer spin diffusion discussed in Sec. IV.B. To be specific, we take both the charge and spin currents to vanish on the outer boundaries of $F_{s}$ and $F_{h}$. It can then be shown (Tserkovnyak et al., 2003b) that the longitudinal spin current flowing into a ferromagnetic slab of thickness $d$ is governed by an effective conductance $g^{*}$ defined by

$$
\frac{1}{g^{*}}=\frac{\tilde{g}^{\uparrow \uparrow}+\tilde{g}^{\downarrow \downarrow}}{2 \tilde{g}^{\uparrow \uparrow} \tilde{g}^{\downarrow \downarrow}}+\frac{1}{g_{\mathrm{sd}} \tanh \left(d / \lambda_{\mathrm{sd}}\right)},
$$

where $g_{\text {sd }}=\left(h / e^{2}\right)\left(S / \lambda_{\text {sd }}\right) 2 \sigma^{\uparrow} \sigma^{\downarrow} /\left(\sigma^{\uparrow}+\sigma^{\downarrow}\right)$ and $\sigma^{s}$ is the spin- $s$ conductivity of the ferromagnetic bulk, so that

$$
\mathbf{I}_{s i}^{(0)}=-\frac{1}{4 \pi}\left[g^{*} \mathbf{m}_{i}\left(\boldsymbol{\mu}_{s N} \cdot \mathbf{m}_{i}\right)+\tilde{g}_{r}^{\uparrow \downarrow} \mathbf{m}_{i} \times \boldsymbol{\mu}_{s N} \times \mathbf{m}_{i}\right] .
$$

The transverse spin current is determined simply by $\tilde{g}_{r}^{\uparrow \downarrow}$ since we have taken $\lambda_{\mathrm{sc}}$ to be the shortest relevant length scale in the problem. Note that $g^{*} \rightarrow 0$ when $d \ll \lambda_{\text {sd }}$, i.e., when the spin-flip relaxation vanishes, or when the ferromagnet is half metallic so that it completely blocks the longitudinal spin flow for a vanishing charge flow. A new parameter

$$
\nu=\frac{\tilde{g}_{r}^{\uparrow \downarrow}-g^{*}}{\tilde{g}_{r}^{\uparrow \downarrow}+g^{*}}
$$

characterizes the asymmetry of the absorption of transverse versus longitudinal spin currents. Putting together Eqs. (92) and (95) and demanding conservation of angular momentum in the spacer (i.e., $\mathbf{I}_{s 1}+\mathbf{I}_{s 2}=\mathbf{0}$ ), one arrives (after some algebra) at

$$
\mathbf{I}_{s 1}^{\text {exch }}=\frac{1}{2}\left[\mathbf{I}_{s 1}^{\text {pump }}-\nu\left(\mathbf{I}_{s 1}^{\text {pump }} \cdot \mathbf{m}_{2}\right) \frac{\mathbf{m}_{2}-\nu \mathbf{m}_{1} \cos \theta}{1-\nu^{2} \cos ^{2} \theta}\right],
$$

where $\cos \theta=\mathbf{m}_{1} \cdot \mathbf{m}_{2}$. Since the normal spacer was taken to be ballistic, $\boldsymbol{\mu}_{s N}$ is uniform. The exchange spin current would otherwise be somewhat suppressed by the spacer diffuse scattering, which can be taken into account easily by solving the spin-diffusion equation as in Sec. IV.B, if necessary. Let us estimate typical values of $\nu$ for sputtered $\mathrm{Cu} \mid \mathrm{Co}$ and $\mathrm{Cu} \mid \mathrm{Py}$ systems at low temperatures, taking $d=5 \mathrm{~nm}$. The main difference between the two material combinations is the spin-diffusion length in ferromagnets. Co has a relatively long $\lambda_{\text {sd }} \approx 60 \mathrm{~nm}$, while $\lambda_{\text {sd }} \approx 5 \mathrm{~nm}$ is very short in Py (Piraux et al., 1996; Bass and Pratt, 1999; Fert and Piraux, 1999). Using known values for spin-dependent conductivities (Piraux et al., 1996; Bass and Pratt, 1999; Fert and Piraux, 1999), we obtain $g_{\text {sd }} / S \approx 2.7 \mathrm{~nm}^{-2}$ for Co and $16 \mathrm{~nm}^{-2}$ for Py. $\left[2 \tilde{g}^{\uparrow \uparrow} \tilde{g}^{\downarrow \downarrow} /\left(\tilde{g}^{\uparrow \uparrow}+\tilde{g}^{\downarrow \downarrow}\right)\right] / S \approx 20 \mathrm{~nm}^{-2}$ for the $\mathrm{Cu} \mid \mathrm{Co}$ interface, see Table I. We may expect the value for $\mathrm{Cu} / \mathrm{Py}$ to be similar. With $\tilde{g}_{r}^{\uparrow \downarrow} / S \approx 27 \mathrm{~nm}^{-2}$ for $\mathrm{Cu} \mid \mathrm{Co}$, see Table I, and $15 \mathrm{~nm}^{-2}$ for $\mathrm{Cu} \mid \mathrm{Py}$ (Bauer et al., 2003b), one finds $\nu$ $\approx 0.98$ for $\mathrm{Cu} \mid \mathrm{Co}$ and $\nu \approx 0.33$ for $\mathrm{Cu} \mid \mathrm{Py}$.

Magnetization dynamics in the absence of an applied bias are determined by substituting $\mathbf{I}_{s}^{\text {exch }}$ into Eq. (14), which thus becomes inconsistent with a constant effective Gilbert parameter. We now analyze the configuration dependence of damping in more detail, which can be measured, in principle, by the FMR linewidth broadening at high rf intensities (and therefore finite "precession cones"). For $\mathbf{m}_{1}$ precessing around $\mathbf{m}_{2}$,

$$
\mathbf{m}_{1} \times \mathbf{I}_{s 1}^{\text {exch }} \times \mathbf{m}_{1}=\frac{1}{2}\left(1-\frac{\nu \sin ^{2} \theta}{1-\nu^{2} \cos ^{2} \theta}\right) \mathbf{I}_{s 1}^{\text {pump }}
$$

The angular dependence of the additional Gilbert damping parameter due to the exchange spin current then reads (Tserkovnyak, et al., 2003b)

$$
\frac{\alpha^{\prime}(\theta)}{\alpha^{\prime}(0)}=1-\frac{\nu \sin ^{2} \theta}{1-\nu^{2} \cos ^{2} \theta},
$$

where $\alpha^{\prime}(0)=\gamma \hbar \tilde{g}_{r}^{\uparrow \downarrow} / 8 \pi M_{s} d S$ is the damping enhancement in a collinear configuration; see Eq. (83). For small angles, $\theta \approx 0$, Eq. (99) can be rewritten as

$$
\frac{\alpha^{\prime}(\theta)}{\alpha^{\prime}(0)} \approx \frac{1}{1+s(1-\cos \theta)}
$$

where

$$
s=\frac{\nu}{1-\nu}
$$

Equation (100) was also obtained by Berger (2001) in the limit of small precession angles, however, for a definition of $s$ with $s \propto \tau_{\text {sf }}$ differing from Eq. (101). Expressions (99) and (100) are plotted in Fig. 18.

As mentioned above, $\nu$ is close to 0.98 for cobalt so that the lower solid line in Fig. 18 represents the damping for Co according to Eq. (99). $s=333$ was found by Berger (2001) for $\mathrm{Cu} \mid \mathrm{Co}$ with $\mathrm{Co} 1.5 \mathrm{~nm}$ thick, which is 


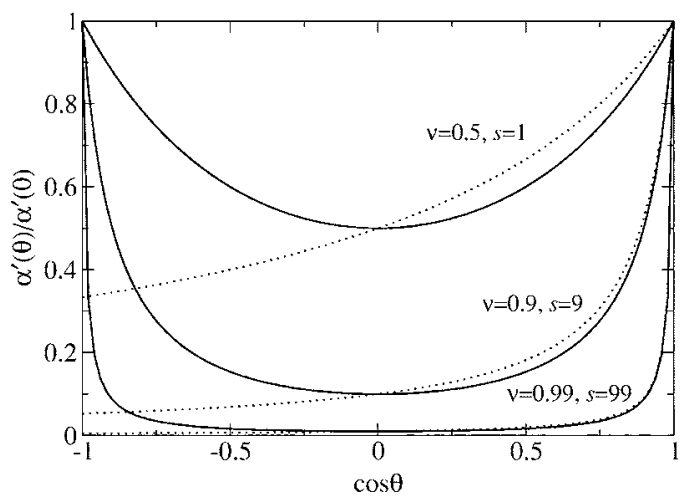

FIG. 18. Solid lines display the precession-cone angle dependence of the Gilbert damping parameter, Eq. (99), and the dotted lines show the extrapolated small-angle approximation (100). The lowest lines are representative for Co and the uppermost ones for Py, assuming magnetic-film thickness of $5 \mathrm{~nm}$. Fe and $\mathrm{Ni}$ are expected to be intermediate between these two cases.

remarkably similar to the estimate based on $\nu$ for this thickness when substituted into Eq. (101). Damping is thus predicted to be significantly reduced for precession angles deviating only slightly from the collinear configurations (we expect this conclusion to also be true for $\mathrm{Fe}$ and $\mathrm{Ni}$ ). Modeling the magnetization dynamics with a constant damping parameter is therefore not allowed for sufficiently thin magnetic layers. For Permalloy, on the other hand, magnetization damping is expected to remain significant at all angles; see the upper solid line in Fig. 18. This implies that the magnetization reversal by an applied magnetic field should be faster in Py than in Co spin valves.

If $\mathbf{m}_{1}$ rotates around an axis perpendicular to $\mathbf{m}_{2}$, i.e., only the relative angle $\theta$ changes, then $\mathbf{I}_{s 1}^{\text {pump }} \perp \mathbf{m}_{2}$ and Eq. (97) reduces to $\mathbf{I}_{s 1}^{\text {exch }}=\mathbf{I}_{s 1}^{\text {pump }} / 2$. The corresponding damping $\alpha^{\prime}(0)$ thus has an angle-independent enhancement with respect to the intrinsic Gilbert damping.

An applied current bias is an additional instrument for controlling magnetization dynamics. When the conductance parameters of the spin valve are mirror symmetric, the bias-induced spin transfer $\mathbf{I}_{s 1}^{\text {bias }}$ is coplanar with the magnetization directions and can be written as

$$
\mathbf{I}_{s 1}^{\text {bias }}=I_{s}^{\text {bias }}(\cos \theta) \frac{\mathbf{m}_{1}+\mathbf{m}_{2}}{2 \cos (\theta / 2)}
$$

for a given applied bias. This follows from expanding the spin current as

$$
\mathbf{I}_{s 1}^{\text {bias }}=f_{11}(\cos \theta) \mathbf{m}_{1}+f_{22}(\cos \theta) \mathbf{m}_{2}+f_{12}(\cos \theta) \mathbf{m}_{1} \times \mathbf{m}_{2}
$$

and noting that $\mathbf{I}_{s 1}^{\text {bias }}\left(\mathbf{m}_{1}, \mathbf{m}_{2}\right)=\mathbf{I}_{s 1}^{\text {bias }}\left(\mathbf{m}_{2}, \mathbf{m}_{1}\right)$ by symmetry, which implies that $f_{11}=f_{22}$ and $f_{12}=0$. The electric current necessary to induce a given spin current $\mathbf{I}_{s}^{\text {bias }}$ depends on $\theta$ and can be calculated readily with circuit theory summarized in Sec. II.A. Equations (14), (91), and (97), and the form of the bias current completely determine the dynamics of $\mathbf{m}_{1}(t)$. The exchange induced by the spin pumping causes relaxation toward an equilibrium configuration, while the bias current can either relax or excite the magnetization, depending on the sign of the current, as discussed below.

Near the parallel configuration, $\theta \approx 0$, Eq. (97) simplifies to $\mathbf{I}_{s 1}^{\text {exch }}=\mathbf{I}_{s 1}^{\text {pump }} / 2$. Let $\mathbf{m}_{1}$ circularly precess around a fixed $\mathbf{m}_{2}$ with the frequency $\omega$ (determined by effective fields): $\mathbf{m}_{1} \times \dot{\mathbf{m}}_{1}=\omega \mathbf{m}_{1} \times \mathbf{m}_{2} \times \mathbf{m}_{1}$. The total projected spin current has the Gilbert form

$$
\mathbf{m}_{1} \times \mathbf{I}_{s 1} \times \mathbf{m}_{1}=\left(\frac{\hbar \tilde{g}_{r}^{\uparrow \downarrow}}{8 \pi}+\frac{I_{s}^{\text {bias }}}{2 \omega}\right) \mathbf{m}_{1} \times \frac{d \mathbf{m}_{1}}{d t} .
$$

Instability is reached when the effective Gilbert damping coefficient becomes negative. The critical bias is thus given by

$$
I_{s, \text { crit }}^{\text {bias }}=-\left(\frac{\tilde{g}_{r}^{\uparrow \downarrow}}{4 \pi}+\frac{2 \alpha M_{s} S d}{\hbar \gamma}\right) \hbar \omega,
$$

where $\alpha$ is the intrinsic Gilbert constant. Neglecting the first term in the brackets, one obtains a result analogous to that of Slonczewski $(1996,1999)$, while neglecting the second term leads to a condition similar to the resonant spin-wave emission criterion (Berger, 1996). The spinpumping contribution (the first term) is comparable to the intrinsic damping (the second term) for transitionmetal films with thickness $d$ of several nanometers, but dominates for very thin films. When the instability is reached, the trajectory of $\mathbf{m}_{1}(t)$ can become very complicated and could possibly lead to a magnetization reversal to a different (meta)stable configuration. A more complete discussion of spin torques and macrospin switching dynamics in asymmetric spin valves can be found in the articles by Manschot et al. (2004) and Brataas, Bauer, and Kelly (2005). Nonlinear large-angle dynamics and field-induced switching in the presence of spin pumping were discussed by Kim and Chappert (2005) within the macrospin model. Nonuniform dynamics have been studied by Brataas, Tserkvovnyak, and Bauer (2005). Experimental evidence for a spinpumping-induced damping in current-driven spin valves has been reported by Krivorotov et al. (2005). The importance of the spin-pumping contribution to the critical current (105) has been investigated experimentally by Sun et al. (2005) for $\mathrm{Co}|\mathrm{Cu}| \mathrm{Co}$ spin valves.

\section{LINEAR-RESPONSE APPROACH}

\section{A. Heterostructures}

This subsection is devoted to an alternative description of the spin emission by a dynamic ferromagnetic magnetization embedded in a conducting nonmagnetic matrix, as in Fig. 2, which was put forward by Šimánek and Heinrich (2003) and further elaborated by Mills (2003) and Šimánek (2003, 2004). In Sec. III we have formulated such spin emission as a scattering pumping process, which requires the concept of waveguide leads for electron states that are reflected by or transmitted through a ferromagnetic layer between normal reser- 
voirs. The time dependence of the scattering matrix caused by a moving magnetization leads to the pumping of spin currents, and the corresponding loss of angular momentum by the ferromagnet to an increased viscous damping of the magnetization dynamics. Such language is standard in the field of mesoscopic transport phenomena (Beenakker, 1997), but the magnetism community is more accustomed to linear-response susceptibilities than to scattering matrices. Of course, the final result should not depend on the formalism used, as verified in the following, but one may argue that the scattering theory has distinct advantages over the linear-response approach for the present problem.

For a linear-response formulation, we proceed from a Hamiltonian for conducting electrons experiencing a ferromagnetic exchange field as given by Eqs. (49) and (50). When the exchange field $V_{x}=-\hbar \Omega / 2$ is uniform inside the ferromagnetic volume $V$ and vanishes outside, we may rewrite Eq. (50) as

$$
H^{\prime}(t)=-\frac{\Omega}{M_{S}} \int_{V} d^{3} r[\mathbf{s}(\mathbf{r}) \cdot \mathbf{M}(\mathbf{r}, t)]
$$

Here $\mathbf{s}(\mathbf{r})$ is the spin-density operator for conduction electrons that are polarized by the exchange field with strength $\Omega$ along the magnetization direction $\mathbf{m}=\mathbf{M} / M_{s}$. $\mathbf{M}$ is a collective property that is treated as a classical time-dependent potential. The Hamiltonian (106) follows, e.g., from a mean-field approximation of the $s-d$ model. The exchange is then viewed as an external potential (provided by the $d$ electrons) that does not depend on the $s$-electron distribution. The spin-densityfunctional formulation of the magnetization dynamics in itinerant ferromagnets, see, e.g., Capelle et al. (2001) and Qian and Vignale (2002), leads to the Hamiltonian (106) in the local-density approximation. The effective field (7) due to the interaction (106) then reads

$$
\mathbf{H}_{\mathrm{eff}}^{\prime}(\mathbf{r}, t)=\frac{\Omega}{M_{s}}\langle\mathbf{s}(\mathbf{r})\rangle_{t} .
$$

In the $s$ - $d$ model, this field corresponds to the reaction torque by the nonequilibrium conduction-electron-spin distribution inside the ferromagnet, which is excited by the moving magnetization direction.

Šimánek and Heinrich (2003) have suggested calculating the reaction torque on the ferromagnetic magnetization directly using Eq. (107) in the Landau-LifshitzGilbert equation of motion. This appears to be very different from the spin-pumping picture that relies on spin currents that are emitted at the interface to the normal metal. The two approaches are, however, related by the continuity equation for electron-spin dynamics (see, e.g., Capelle et al., 2001), according to which the spin current equals the torque exerted on the magnetization $\mathbf{M}$ by the effective field (107) up to a term given by the time derivative of the average spin density, $\langle\dot{\mathbf{s}}\rangle_{t}$, times volume. When $\langle\mathbf{s}\rangle_{t}$ follows the magnetization adiabatically, the difference between the spin current and torque is thus proportional to $\mathbf{m}$. In the case of the $s-d$ model, this has a physical meaning, corresponding to the torque required to change the angular momentum of the $s$-electron spin density. For Stoner ferromagnets in which the conduction electrons are identical to the ones that make up the magnetization, we have to calculate the spin flow emitted by the ferromagnet as an additional term in the Landau-Lifshitz-Gilbert equation, while the reaction torque on the magnetization by the conduction electrons has no obvious physical meaning. When treating itinerant (Stoner) ferromagnets within the density-functional theory, the spin currents induced by the Kohn-Sham Hamiltonian (106) in principle differ from the physical ones inside or very close to the ferromagnet. However, this difference vanishes asymptotically as a function of distance from the ferromagnet (Capelle et al., 2001). The emitted spin currents are thus similar to those in the $s$ - $d$ model, and their evaluation can thus be mapped on calculating corresponding reaction torques.

A uniform small-angle dynamics (of a possibly large $\Omega$ ) only weakly perturbs the system. The induced spin imbalance $\langle\delta \mathbf{s}(\mathbf{r})\rangle_{t}$ can therefore be expressed in terms of the linear-response susceptibility of the unperturbed system,

$$
\chi_{s_{a^{a^{\prime}}}}(t)=\frac{i}{\hbar V} \Theta(t) \int_{V} d^{3} r d^{3} r^{\prime}\left\langle\left[s_{a}(\mathbf{r}, t), s_{a^{\prime}}\left(\mathbf{r}^{\prime}, 0\right)\right]\right\rangle,
$$

where $\Theta(t)$ is the Heaviside step function. The effective field due to the induced nonequilibrium spin density is then given by

$$
\begin{aligned}
\delta \mathbf{H}_{\mathrm{eff}}^{\prime}(t) & =\frac{\Omega}{M_{S} V} \int_{V} d^{3} r\langle\delta \mathbf{s}(\mathbf{r})\rangle_{t} \\
& =\frac{\Omega}{M_{s}} \sum_{a a^{\prime}} \int_{-\infty}^{\infty} d t^{\prime} \times \hat{\mathbf{a}}{\chi_{a_{a} a^{\prime}}}\left(t-t^{\prime}\right) \delta m_{a^{\prime}}\left(t^{\prime}\right),
\end{aligned}
$$

where $a, a^{\prime} \in\{x, y, z\}$ are the indices of the Cartesian axes and $\hat{\mathbf{a}}$ stands for the corresponding unit vectors. Suppose now for simplicity that the system is invariant under spin rotations about the $z$ axis, and consider small-angle magnetization dynamics near this axis, $\delta \mathbf{m}$ $=\mathbf{m}-\hat{\mathbf{z}}$. Substituting Eq. (109) into the Landau-LifshitzGilbert equation (8) then yields the following lowestorder dynamic term:

$$
-\gamma \mathbf{m} \times \delta \mathbf{H}_{\mathrm{eff}}^{\prime}(t)=\frac{\gamma \Omega^{2}}{M_{s}}\left(\Lambda_{1} \frac{d \mathbf{m}}{d t}+\Lambda_{2} \mathbf{m} \times \frac{d \mathbf{m}}{d t}\right)
$$

which is the most general adiabatic term for axially symmetric systems (Mills and Rezende, 2003). Here

$$
\Lambda_{1}=-\left.i \frac{d \chi_{s_{x} s_{y}}(\omega)}{d \omega}\right|_{\omega=0},
$$




$$
\Lambda_{2}=-\left.i \frac{d \chi_{s_{x} s_{x}}(\omega)}{d \omega}\right|_{\omega=0}
$$

are real numbers. $\Lambda_{1}$ renormalizes the effective gyromagnetic ratio $\gamma_{\text {eff }}$ in Eq. (8) and $\Lambda_{2}$ is a Gilbert-like damping parameter,

$$
\alpha_{\mathrm{eff}}=-\left.i \frac{\gamma_{\mathrm{eff}} \Omega^{2}}{M_{s}} \frac{d \chi_{s_{x} s_{x}}(\omega)}{d \omega}\right|_{\omega=0} .
$$

Equation (112) can also be obtained by equating the energy dissipated into the itinerant degrees of freedom by moving magnetization and the work done by an rf magnetic field applied against the effective viscous Gilbert term, at a steady magnetic precession, since $\lim _{\omega \rightarrow 0} \operatorname{Re} \chi_{s_{x} s_{y}}(\omega) / \omega=0$ in the case of spin-rotational symmetry around the $z$ axis. For a thermally equilibrated $s$-electron subsystem, Eq. (112) implies $\alpha_{\text {eff }} \gamma_{\text {eff }}$ $>0$, as required by the Landau-Lifshitz-Gilbert phenomenology; see Sec. I.C.

The damping constant $\alpha$ can generally be expressed in terms of the response function of the total magnetization (e.g., of the $s$ and $d$ electrons in the $s$ - $d$ model) by inverting Eq. (11). In the present discussion, this problem is self-consistently reduced to the simpler task of calculating the spin response of $s$ electrons to a timedependent exchange field aligned with the $d$-electron magnetization.

The similarity between the torque (110) and the spinpumping current (38) should not be surprising in light of the above discussion. Indeed, Šimánek (2003) explicitly demonstrated that the low-frequency linear-response and spin-pumping pictures lead to identical $\alpha_{\text {eff }}$ for a $\delta$-function magnetic layer embedded in a clean normal metal (which corresponds to an ideal spin sink for emitted spin currents in the spin-pumping language). However, evaluating the linear-response correlation functions becomes very tedious for more realistic models; see, e.g., Mills (2003). It is also not obvious how to treat the current-induced spin transfer and the spin pumping on the same footing with the linear-response formalism. On the other hand, the formulation in terms of the susceptibilities is complementary to the spin-pumping approach. It appeals to the intuition of many researchers in the magnetism community. Furthermore, it could be helpful for obtaining insights into problems that are hard to solve within scattering theory. Examples of these are magnetic bilayers coupled by a strong static exchange interaction (see Sec. VII.A.2), strongly correlated systems (Sec. VII.D), and the bulk damping of magnetization dynamics (Sec. VI.B).

\section{B. Bulk damping}

In the previous subsection, we have considered coupling of the magnetization to itinerant electrons via an exchange interaction (106). In particular, it was reasserted that when a ferromagnetic film is inserted into a nonmagnetic metal, magnetization dynamics causes an emission of spins. In the presence of a spin sink outside the ferromagnet, this pumping leads to a net angularmomentum loss that is equivalent to an excess damping of the magnetization dynamics, as discussed in Sec. IV. An analogous mechanism could be effective in bulk ferromagnets provided that spin-relaxation decay channels exist that dissipate a spin accumulation created by the pumping. This happens in the presence of momentumscattering mechanisms, such as lattice impurities and phonons, and the accompanying (or band-structure) relativistic spin-orbit interaction. The inductive coupling of the magnetization to the conduction electrons that causes dissipation by eddy currents is less important in thin layered structures and is disregarded in the following. For simplicity, the discussion is restricted in the following to a mean-field $s-d$ model.

A mechanism of bulk magnetization damping bearing similarity to the spin-pumping picture in heterostructures was proposed a long time ago by Mitchell (1957). It involves a transfer of the angular momentum (and energy) of a nonequilibrium ferromagnetic configuration to the itinerant electrons via the exchange interaction, with a subsequent spin-orbit relaxation to the lattice. The consequences of such a process for macrospin (longwavelength) dynamics have been worked out for the $s$ - $d$ model by Heinrich et al. (1967). The $s$ - $d$ picture has been resurrected recently by Sinova et al. (2004) in order to address the magnetization relaxation in the ferromagnetic semiconductor $(\mathrm{Ga}, \mathrm{Mn}) \mathrm{As}$, in which ferromagnetism originates from the free-hole-mediated exchange interaction between dilute, substitutional spin-5/2 Mn atoms (Ohno, 1999). It is possible to reproduce and generalize the results of Heinrich et al. (1967) and Sinova et al. (2004) by calculating the magnetization damping in terms of the conduction-electron-spin dynamics in a time-dependent exchange field similarly to Sec. VI.A (Tserkovnyak et al., 2004).

Consider an $s p$ - $d$ model of a conducting ferromagnet, where the spin $\mathbf{S}$ of the itinerant $s$ or $p$ states (either electrons or holes) experiences an exchange field of magnitude $\Omega$ along the uniform magnetization direction $\mathbf{m}$ of the localized $d$ orbitals, as in Eq. (106):

$$
H(t)=H_{0}-\Omega \mathbf{m}(t) \cdot \mathbf{S} .
$$

Here $H_{0}$ is a one-particle Hamiltonian reflecting the host band structure. In (Ga,Mn)As, substitutional $\mathrm{Mn}$ are paramagnetic acceptors that strongly interact with the free holes. Although the exchange field can be highly nonuniform on atomic scales, it is customary to start with a simplifying assumption that it may be smeared out. As before, we treat the magnetization $\mathbf{m}$ as a classical and, on the relevant length scales of the carrier dynamics, spatially uniform variable. A suitable model for the valence bands of a dilute $p$-doped semiconductor (e.g., GaAs, $\mathrm{Si}$, or $\mathrm{Ge}$ ) is the spherical Luttinger Hamiltonian for spin-3/2 holes: 


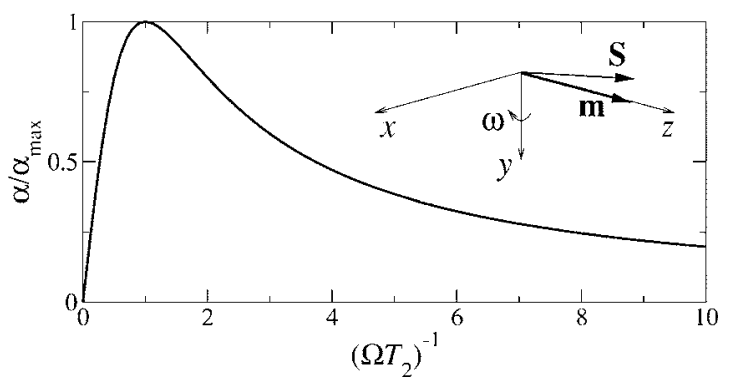

FIG. 19. Gilbert damping, Eq. (116), in units of $\alpha_{\max }$ $=\gamma s_{0} / 2 M_{s}$ as a function of the normalized spin-relaxation rate. Inset: Geometry of the model.

$$
H_{0}=\frac{1}{2 m_{e}}\left[\left(\gamma_{1}+\frac{5}{2} \gamma_{2}\right) p^{2}-2 \gamma_{2}\left(\frac{\mathbf{p} \cdot \mathbf{S}}{\hbar}\right)^{2}\right],
$$

where $m_{e}$ is the free-electron mass and $\gamma_{i}$ are the Luttinger parameters (Luttinger, 1956). The spin-orbit term couples the hole momentum $\mathbf{p}$ with its spin $\mathbf{S}$. The fourband model (114) is valid when the hole Fermi energy is sufficiently smaller than the spin-orbit splitting of the semiconductor host between the spin-3/2 and spin-1/2 valence bands. Equation (114) neglects corrections for lattices with broken inversion symmetry. Spin-1/2 electron systems with vanishing spin-orbit coupling are recovered by setting $\gamma_{2}=0$. Suppose the magnetization of the localized orbitals varies slowly in time (being uniform at all times) so that the time-dependent $\mathbf{m}$ modulates the Hamiltonian (113) adiabatically. This means that the system equilibrates on time scales faster than the motion of $\mathbf{m}$ and all the quantities parametrizing the carrier Hamiltonian remain constant. The observation of ferromagnetic resonance indicates that time-dependent long-range ferromagnetic order indeed exists in thin films of magnetic transition metals (Heinrich and Cochran, 1993) and semiconductors (Goennenwein et al., 2003; Rappoport et al., 2004). Equation (112) may then be taken as a microscopic definition of the Gilbert damping parameter. In the following, $\alpha$ is formally evaluated for electron and hole systems with an emphasis on its dependence on spin dephasing. (We shall drop the subscripts "eff" on $\alpha$ and $\gamma$ from the previous subsection.)

We initially focus on a system without spin-orbit coupling in the band structure, $\gamma_{2}=0$. Suppose the transverse spin-density dynamics in the exchange field $\Omega$ is described by the Bloch equation

$$
\frac{d \mathbf{s}}{d t}=\Omega \mathbf{s} \times \mathbf{m}(t)-\frac{\mathbf{s}-s_{0} \mathbf{m}(t)}{T_{2}},
$$

where the last term is a phenomenological relaxation due to impurities, parametrized by the transverse dephasing time $T_{2}$. Let us assume that $\mathbf{m}(t)$ moves slowly on the scales of $\Omega^{-1}$ and $T_{2}$. It is then convenient to transform Eq. (115) into a frame of reference (for spin variables) that moves together with $\mathbf{m}(t)$. When, for example, $\mathbf{m}$ at a given instant rotates with frequency $\omega$ around the $y$ axis (see inset of Fig. 19), it is stationary in the rotating frame at the cost of a new Larmor term $\omega \mathbf{s} \times \hat{\mathbf{y}}$ on the right-hand side of Eq. (115), which corresponds to a magnetic field along the $y$ axis. Since the motion is slow, it is sufficient to solve for $\mathbf{s}$ as the instantaneous stationary state in the moving frame of reference. If $\mathbf{m}$ points along the $z$ axis in the rotating frame, spin polarization along the $x$ axis then exerts a damping torque on $\mathbf{m}$ that corresponds to

$$
\alpha=-\frac{\gamma \tilde{\chi}_{s_{x} s_{y}} \Omega}{M_{s}}=\frac{\Omega T_{2}}{1+\left(\Omega T_{2}\right)^{2}} \frac{\gamma s_{0}}{M_{s}},
$$

where $\tilde{\chi}_{s_{x} s_{y}}$ is the stationary (real-valued) response function in the rotating frame. (We recall that $\gamma$ is the gyromagnetic ratio of $d$ orbitals.) The calculation of the timedependent linear response in the laboratory frame, Eq. (112), is thus simplified to that of the static response in the rotating frame. Such a transformation is, however, not possible in general, in particular for Hamiltonians that are not spin rotationally invariant, such as Eq. (113). Equation (116) is plotted in Fig. 19. The equilibrium spin density $s_{0}$ can be calculated from the specific form of the static Hamiltonian. $\alpha$ vanishes at both small and large spin-relaxation limits.

The damping parameter (116) depends only on the ratio of spin-relaxation-rate and exchange energy. The low spin-relaxation-rate regime, $\alpha \propto T_{2}^{-1}$, is analogous to the spin-pumping damping of thin films in contact with a spin-sink conductor. The moving magnetization "pumps" spins into the itinerant carriers at a constant rate, which are then relaxed with a probability $\propto T_{2}^{-1}$ before exchanging spins with the ferromagnet again. The difference is that now the spins are pumped into the internal conduction electrons of the ferromagnet rather than those of an externally attached metal. The other limit, $\alpha \propto T_{2}$, can be understood by noting that for viscous dynamics of $\mathbf{s}(t), \mathbf{s}(t) \approx \mathbf{s}_{0}(t)-T_{2} \dot{\mathbf{s}}_{0}(t)$ in the laboratory frame. $\alpha \propto T_{2}$ then follows from the torque $\propto(\mathbf{s}$ $\left.-\mathbf{s}_{0}\right) \times \mathbf{m}$. This is analogous to the "breathing Fermisurface" damping mechanism discussed by Kunes and Kamberský (2002) in the regime of fast relaxation. The itinerant carriers try to lower their energy by rearranging themselves in the field of slowly varying magnetization direction but lag behind with a short delay time determined by relevant relaxation processes. In the presence of an anisotropic spin-orbit interaction in the ferromagnet's crystal field, the breathing Fermi surface gives an additional contribution to damping (Kunes and Kamberský, 2002). See also a short discussion in Sec. VII.A.2. In the present model described by Eq. (115), the breathing takes place in spin space.

It is interesting to note that Eq. (116) reduces to the result obtained by Heinrich et al. (1967) for the longwavelength magnon lifetime due to the $s-d$ interaction with spin- $1 / 2$ conduction electrons in the random-phase approximation: 


$$
\alpha=\frac{\Omega T_{2}}{1+\left(\Omega T_{2}\right)^{2}} \frac{\gamma\left[\Omega m^{*} k_{F} / 4 \pi^{2} \hbar\right]}{M_{s}},
$$

where $m^{*}$ is the band-structure mass and $k_{F}$ the Fermi wave vector. Here it is assumed that $\hbar \Omega \ll E_{F}$ (the Fermi energy). The quantity in the square brackets is just the total carrier spin density. Equation (117) was used by Ingvarsson et al. (2002) in order to explain the measured damping in thin Permalloy films, which scaled linearly with the film resistivity $\rho$. A linear relation between the two quantities is expected when $\Omega \gg T_{2}^{-1}$, so that $\alpha$ $\propto T_{2}^{-1}$, and using the Drude formula $\rho \propto \tau^{-1}$ ( $\tau$ is the transport mean free time) and assuming $\tau / T_{2}=$ const, which depends on material and scattering-impurity type but not on scattering rate (Abrikosov and Gor'kov, 1962). Unlike ferromagnetic semiconductors, however, the use of the $s-d$ model for itinerant ferromagnets such as transition metals is questionable, since the separate treatment of magnetic and conduction electrons is unphysical.

Let us turn our attention to the magnetization relaxation in hole-doped magnetic semiconductor (Ga, Mn)As. Equation (116) may be used to obtain a rough estimate of the damping coefficient. The largest achievable value of $\alpha_{\max }=\gamma s_{0} / 2 M_{s}$ occurs when the holes are fully polarized, giving $\alpha_{\max } \sim 0.1-0.3$, roughly one-third the ratio of the (spin-3/2) hole to the substitutional (spin-5/2) Mn concentrations. For realistic samples with a spin polarization of the order of unity therefore $\alpha_{\max }$ $\sim 0.1$. The damping $\alpha$ is further suppressed by $\alpha / \alpha_{\max }$ $=2 \Omega T_{2}\left[1+\left(\Omega T_{2}\right)^{2}\right]^{-1}<1$. For clean bulk samples of GaAs, the spin-relaxation time is $\sim 100$ fs (Hilton and Tang, 2003). For approximately $5 \% \mathrm{Mn}$ doping, $\hbar \Omega$ $\sim 0.1 \mathrm{eV}$ (Dietl et al., 2001), so that $\Omega T_{2} \sim 10$. This corresponds to the $\alpha \propto T_{2}^{-1}$ regime with $\alpha \sim 0.01$. Reduced spin-relaxation times should thus result in a larger damping. Experimentally, impurity scattering is likely to be the easiest parameter to vary in order to tune $\alpha$ to a desired value. The strong spin-orbit coupling $\gamma_{2}$, however, makes the validity of the phenomenological equation (115), and thus the result (116), questionable for the hole system. Besides, a strong crystal anisotropy would require a further refinement of the analysis. Returning to our basic equation (112) and inserting the response function for a noninteracting system yields

$$
\begin{aligned}
\alpha= & \frac{\gamma \Omega^{2}}{M_{s} V} \lim _{\omega \rightarrow 0} \frac{\pi}{\omega} \sum_{i j}\left|\left\langle i\left|S_{x}\right| j\right\rangle\right|^{2}\left[f_{\mathrm{FD}}\left(\varepsilon_{i}\right)-f_{\mathrm{FD}}\left(\varepsilon_{j}\right)\right] \\
& \times \delta\left(\hbar \omega+\varepsilon_{i}-\varepsilon_{j}\right),
\end{aligned}
$$

where $i, j$ label the one-particle eigenstates of the sample with volume $V$. When the wave vector $\mathbf{k}$ is conserved, $\Sigma_{i j} / V=\Sigma_{\sigma \sigma^{\prime}} \int d^{3} k /(2 \pi)^{3}$, where $\sigma, \sigma^{\prime}$ label spin states. For a perfect crystal therefore $\alpha$ vanishes, unless there is a finite Fermi-surface area with spin degenerate states. Introducing lattice defects leads to a finite $\alpha$. See, e.g., Sinova et al. (2004) (these authors did not include important vertex corrections in the response function, however).
In the above analysis, we have assumed a coherent motion of the ferromagnetic magnetization without specifying the source of excitation. An FMR magnetic field (with a large dc and small rf components), for example, can be included explicitly in the Hamiltonian (113) of the itinerant carriers. The results for the Gilbert damping will stay unaffected, however, as long as the exchange energy $\hbar \Omega$ is much larger than the carrier Zeeman splitting in the applied field and ferromagnetic magnetization is mainly carried by localized orbitals. (The energy pumped into carrier-magnetization dynamics by the rf field must be taken into account otherwise.) Inhomogeneities in the bulk magnetization are not important on the length scales set by the transverse spinrelaxation rate and the precession frequency in the exchange field. Bulk spin dynamics discussed in this subsection have no effect on the spin pumping into adjacent conductors discussed in Sec. III, as long as the transverse spin-relaxation rate is small compared to the exchange precession frequency. In the opposite rather unrealistic limit, the $s$-electron spin dynamics are locked to the $d$-electron magnetization motion, suppressing spin leakage (pumping) into adjacent normal conductors.

\section{MISCELLANEOUS}

\section{A. Quantum-size effects}

\section{Ultrathin magnetic layer}

As explained in Sec. III, spin pumping by a magnetic layer in contact with normal metals is governed by the parameter $\mathcal{A}^{\uparrow \downarrow}=g^{\uparrow \downarrow}-t^{\uparrow \uparrow}$, Eq. (39). When the magneticfilm thickness $d$ exceeds the spin-coherence length (13), $d \gg \lambda_{\mathrm{sc}}, t^{\prime \uparrow \downarrow}$ vanishes and $\mathcal{A}^{\uparrow \downarrow}$ is given by the interfacial spin-mixing conductance $g^{\uparrow \downarrow}$. In this subsection, on the other hand, we focus on the regime in which $d$ is smaller than or of the order of $\lambda_{\mathrm{sc}}$, i.e., for thicknesses of a few monolayers in the case of transition-metal ferromagnets. In this limit, the coherence between up- and down-spin states in the ferromagnet leads to a thickness dependence of $\mathcal{A}^{\uparrow \downarrow}$, and thus of spin pumping and magnetization torque (27). The decoherence of the orbital wave function due to inelastic-scattering processes is disregarded, assuming its characteristic length scale is much longer than $\lambda_{\mathrm{sc}}$.

As in most of this review, the structures that we study have multiple layers. A large lateral area $S$ renders mesoscopic phenomena such as the Coulomb blockade irrelevant, and we focus on quantum-coherence effects due to small layer thicknesses. For a study of magnetization dynamics in magnetic nanoparticles in contact with large reservoirs, see, e.g., Waintal and Brouwer (2003) and Waintal and Parcollet (2005).

The linear-response framework (Šimánek and Heinrich, 2003), see also Sec. VI, has been used by Mills (2003) to calculate the enhanced Gilbert damping of finite-thickness ferromagnetic films embedded in a conducting medium. For an idealistic model of isotropic band structure with spin-dependent exchange potential, 


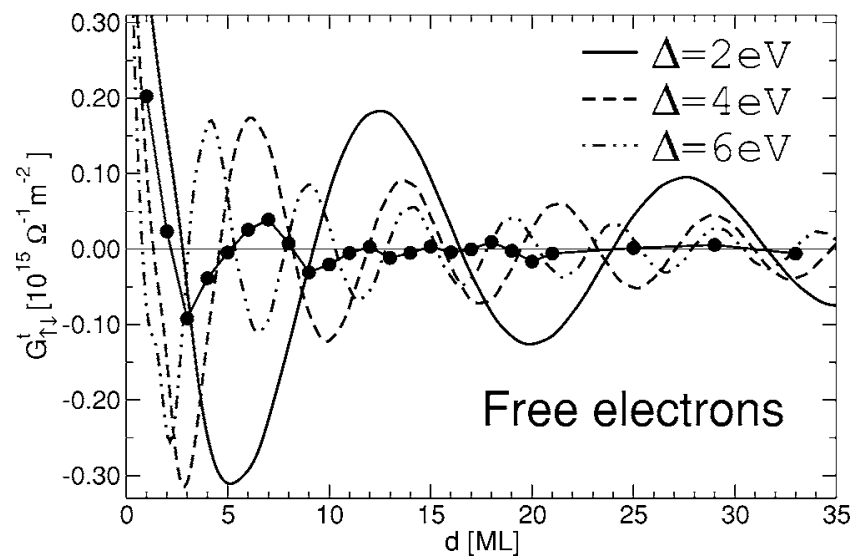

FIG. 20. The real part of $G_{\uparrow \downarrow}^{t}=\left(e^{2} / h\right) t^{\uparrow \downarrow} / S$ as a function of the magnetic-layer thickness $d$ calculated from first principles (O) for realistic multiband electronic structures and that calculated for an isotropic free-electron gas with Fermi energy $\varepsilon_{F}=7 \mathrm{eV}$ (chosen to obtain the correct value of the Sharvin conductance in $\mathrm{Cu}$ ) and various choices of the exchange splitting $\Delta$. From Zwierzycki et al., 2005.

he found that ultrathin films display oscillatory damping (as a function of thickness) due to quantum-size effects. However, by calculating scattering matrices with electronic structures computed in the local-spin-density approximation, Zwierzycki et al. (2005) have shown that quantum-size oscillations are much smaller than those obtained by Mills (2003), and small amounts of disorder suppress the remaining oscillations even further. Zwierzycki et al. (2005) also found that the spin-pumping torque is of the Gilbert damping form, with only a very small correction to the gyromagnetic ratio.

The above observations have been made for copper/ cobalt and gold/iron heterostructures, of which we shall restrict our discussion to the former. Realistic bandstructure and simple model calculations on $\mathrm{Cu}|\mathrm{Co}| \mathrm{Cu}(111)$ trilayers are compared in Fig. 20, where the real part of $G_{\uparrow \downarrow}^{t}=\left(e^{2} / h\right) t^{\uparrow \downarrow} / S$ is plotted as a function of the thickness $d$ of the magnetic layer (measured in atomic monolayers). Solid circles in Fig. 20 show the result of the ab initio calculation (Zwierzycki et al., 2005) without impurities and for specular ( $\mathbf{k}_{\|}$-conserving) interfaces. The smooth solid lines represent the calculations for the isotropic free-electron model. For exchange splittings $\Delta=2,4$, and $6 \mathrm{eV}$ the amplitude of oscillation is much larger and the decay is much slower in the model than in the more realistic first-principles calculations. As might be expected, increasing the exchange splitting from 2 to $6 \mathrm{eV}$ leads to a shorter period and more rapid decay of the oscillations. In order to mimic the parameter-free $a b$ initio result, an exchange splitting of about $10 \mathrm{eV}$, however, would be needed (not shown). Such a large value can be justified neither on theoretical nor experimental grounds. This discrepancy illustrates the difficulty of quantitatively representing the complex electronic structure of transition metals by simple model Hamiltonians.

Figure 21 shows $G_{\uparrow \downarrow}^{r}=\left(e^{2} / h\right) g^{\uparrow \downarrow / S}$ and $G_{\uparrow \downarrow}^{t}$ calculated
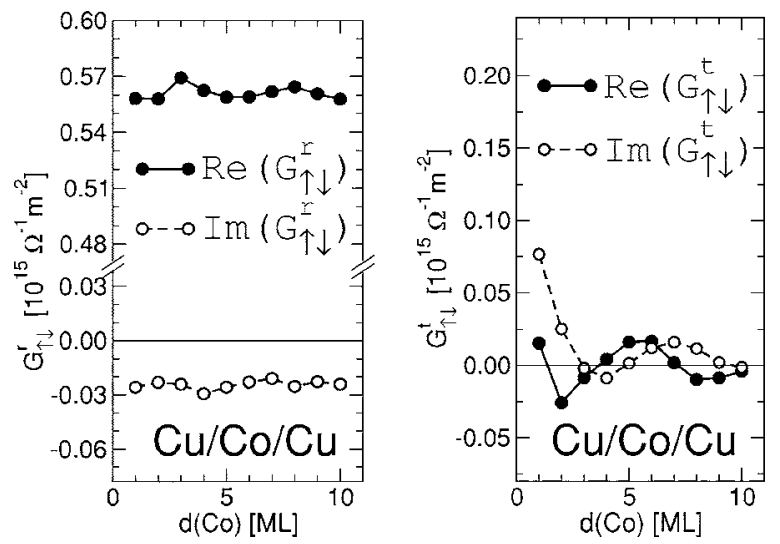

FIG. 21. Spin-mixing conductances of a $\mathrm{Cu}|\mathrm{Co}| \mathrm{Cu}(111)$ structure with disordered interfaces as a function of the Co-layer thickness. From Zwierzycki et al., 2005.

from first principles in the presence of disorder modeled by a monolayer of $50 \%$ alloy added on each side of the magnetic layer. The thickness $d$ in this case is defined as that of the remaining clean ferromagnetic layer. The disorder strongly quenches the amplitudes of the oscillations as a function of $d$, so that $G_{\uparrow \downarrow}^{r}$ is practically constant at the level of its asymptotic (i.e., single-interface) value. For $G_{\uparrow \downarrow}^{t}$, the oscillations do not vanish completely, but their amplitude is substantially reduced to values that are negligibly small compared to $\operatorname{Re} G_{\uparrow \downarrow}^{r}$ for all but the thinnest magnetic layers. Diffusive scattering in the bulk of the magnetic layer, which has not been considered here explicitly, should have similar effects.

Both $G_{\uparrow \downarrow}^{r}$ and $G_{\uparrow \downarrow}^{t}$ are governed by (i) the matching of the normal-metal and ferromagnetic-metal states described by the scattering matrix of the isolated interface and (ii) the phases accumulated by electrons on their quantum-coherent propagation through the magnetic layer. The interface determines the amplitudes of the oscillations and the asymptotic value of $G_{\uparrow \downarrow}^{r}$, whereas the bulk term is responsible for the oscillation period. It is instructive to interpret the transmission and reflection coefficients of the finite-size magnetic layer in terms of multiple scattering between the two interfaces. The task is simplified by the simple Fermi surface of $\mathrm{Cu}$, which corresponds to only one left- and right-going state at the Fermi energy for each value of $\mathbf{k}_{\|}$and spin. The sums over states in Eqs. (23) and (24) therefore reduce to integrations over the two-dimensional Brillouin zone involving the complex-valued functions $r^{\sigma}\left(\mathbf{k}_{\|}\right)$and $t^{\sigma}\left(\mathbf{k}_{\|}\right)$. To lowest order in the number of scattering processes and dropping explicit reference to $\mathbf{k}_{\|}$,

$$
\begin{aligned}
& t^{\sigma} \approx t_{F \rightarrow N}^{\sigma} \Lambda^{\sigma} t_{N \rightarrow F}^{\sigma}, \\
& r^{\sigma} \approx r_{N \rightarrow N}^{\sigma}+t_{F \rightarrow N}^{\sigma} \Lambda^{\sigma} r_{F \rightarrow F}^{\sigma} \Lambda^{\sigma} t_{N \rightarrow F}^{\sigma},
\end{aligned}
$$

where $t_{N \rightarrow F}^{\sigma}=\left(t_{1}^{\sigma}, \ldots, t_{n}^{\sigma}\right)^{T}$ is a vector of transmission coefficients between a single propagating state in the normal metal and a set of states in the ferromagnet, $\Lambda^{\sigma}$ is a diagonal matrix of phase factors $e^{i k_{j \perp}^{\sigma} d}$ ( $j$ is an index of 
the states in the ferromagnet), $r_{N \rightarrow N}^{\sigma}$ is a scalar reflection coefficient for states incoming from the normal metal, and $r_{F \rightarrow F}^{\sigma}$ is a square matrix describing reflection at the ferromagnetic side. The set of states in the ferromagnet consists of both propagating and evanescent states. The contribution of the latter decreases exponentially with the thickness of the layer.

Let us first analyze the thickness dependence of $t^{\uparrow \downarrow}$. After inserting Eq. (119) into Eq. (24) the summation may be carried out over terms containing phase factors $e^{i\left(k_{i \perp}^{\uparrow}-k_{j \perp}^{\downarrow}\right) d}$. Because of the large differences between majority and minority Fermi surfaces of the ferromagnet, this typically leads to rapidly oscillating terms that cancel in the two-dimensional Brillouin-zone integration over $\mathbf{k}_{\|}$. In the spirit of the theory of interlayer exchange coupling (Bruno, 1995; Stiles and Zangwill, 2002), longrange contributions must originate from the vicinity of points for which $\nabla_{\mathbf{k}_{\|}}\left(k_{i \perp}^{\uparrow}-k_{j \perp}^{\downarrow}\right)=0$, corresponding to a constant phase in the integrand of Eq. (24). The damped oscillations around zero are therefore caused by the stationary-phase Fermi-surface calipers.

By substituting Eq. (120) into Eq. (23), two thicknessindependent contributions can be identified in $g^{\uparrow \downarrow}$. The first, coming from integrating the $\delta_{n n^{\prime}}$ term in Eq. (23), is the number of transport channels in the normal metal, i.e., the dimensionless Sharvin conductance. The second contribution comes from the $r_{N \rightarrow N}^{\uparrow} r_{N \rightarrow N}^{\downarrow *}$ term and provides an interface-specific correction to the first contribution. The thickness-dependent contributions contain, to lowest order, phase factors $e^{i\left(k_{i \perp}^{\sigma}+k_{j \perp}^{\sigma}\right) d}$ and $e^{-i\left(k_{i \perp}^{\sigma}+k_{j \perp}^{\sigma}\right) d}$. As in the case of $t^{\uparrow \downarrow}$, their two-dimensional Brillouinzone integrals have an oscillatory character, with periods governed by different Fermi-surface calipers. These oscillations now occur around the constant value set by the first two contributions. The asymptotic value of $g^{\uparrow \downarrow}$ is clearly the reflection mixing conductance of a single $F \mid N$ interface.

In metallic films, the electronic structure of all but the outermost atomic layers is practically identical to that of the bulk material. The period of oscillations of $g^{\uparrow \downarrow}$ and $t^{\uparrow \downarrow}$ as a function of the magnetic-layer thickness $d$ is thus a bulk property of the magnetic layer. The amplitudes, on the other hand, involve interfacial scattering coefficients introduced in Eqs. (119) and (120). When quantum-size oscillations are small, $\mathcal{A}^{\uparrow \downarrow} \approx g^{\uparrow \downarrow}$, where $g^{\uparrow \downarrow}$ is a property of the $N \mid F$ interface instead of the entire structure. Furthermore, $g^{\uparrow \downarrow}$ can be estimated by the Sharvin conductance of the normal metal, Eq. (64). The results of single-interface calculations are listed in Table I for clean and disordered interfaces of $\mathrm{Cu} \mid \mathrm{Co}$ and $\mathrm{Au} \mid \mathrm{Fe}$ material combinations. The disorder in Table I was modeled by 2 monolayers of $50 \%$ alloy instead of a single monolayer in the present subsection. In spite of this difference, the values are practically identical to the asymptotic ones seen in Fig. 21 for the $\mathrm{Cu} \mid \mathrm{Co}$ combination.

\section{Ultrathin normal spacer}

Let us now turn to a discussion of magnetization dynamics of two monodomain magnetic layers separated by ultrathin normal-metal spacers with quantum-well states that penetrate into and couple the ferromagnets. As explained in Sec. I.B, the free energy $F$ of the system depends on the relative angle between the two magnetizations even in the absence of magnetic anisotropies. The dependence of $F\left(\mathbf{M}_{i}\right), i=1,2$, on the magnetic configuration corresponds to nonlocal effective fields (6) exerting torques on the magnetizations. In the following, we comment on the dynamic component of the exchange interaction in time-dependent problems, cf. the semiclassical dynamic exchange interaction discussed in Sec. V.

Consider for simplicity an $s$ - $d$ model with noninteracting $s$ electrons, in which the magnetic $d$ orbitals are coupled to itinerant electrons via a mean-field exchange interaction. The transverse component of the effective field (7) entering the Landau-Lifshitz-Gilbert equation (8) for the $i$ th magnetic moment is then given by

$$
\begin{aligned}
\mathbf{H}_{i}(t) & =-\frac{1}{M_{s i} V_{i}} \partial_{\mathbf{m}_{i}}\left\langle H\left(\mathbf{m}_{i}\right)\right\rangle_{t} \\
& =-\frac{1}{M_{s i} V_{i}} \partial_{\mathbf{m}_{i}} \sum_{\kappa} \varepsilon_{\kappa}\left(\mathbf{m}_{i}\right) n_{\kappa}\left(\mathbf{m}_{i}, t\right),
\end{aligned}
$$

where $H\left(\mathbf{m}_{i}\right)$ is the Hamiltonian for itinerant electrons, parametrized by the magnetization directions $\mathbf{m}_{i}$, and \langle\rangle$_{t}$ denotes the (quantum-mechanical) expectation value at time $t$. The sum on the second line of Eq. (121) runs over all eigenstates of $H\left(\mathbf{m}_{i}\right)$, where $\kappa$ labels both the orbital and spin degrees of freedom, $n_{\kappa}$ is the occupation number corresponding to the many-body state at time $t$, and $\varepsilon_{\kappa}$ is the energy of the $\kappa$ th eigenstate. Setting the manybody ensemble at time $t$ to its equilibrium value determined by $\mathbf{m}_{i}(t)$ reproduces the Landau-Lifshitz definition (6), leading to dissipationless trajectories [assuming there are no other sources of damping, i.e., $\alpha=0$ in Eq. (8)]. Such an approximation thus captures only the static exchange component.

In reality, \langle\rangle$_{t}$ lags behind its instantaneous equilibrium value. The corresponding nonequilibrium component of $n_{\kappa}\left(\mathbf{m}_{i}, t\right)$ in momentum space reflects the breathing Fermi surface discussed by Kunes and Kamberský (2002) in the context of transition-metal bulk magnetization damping in the presence of crystal anisotropy. Heinrich, Urban, et al. (2003) conjectured that such a mechanism may also play a role in magnetic dynamics of $F|N| F$ structures. The modulation of the exchange energy stored in the normal spacer may cause an additional damping through the time lag in the itinerantelectron response.

For a spin rotationally invariant system, the effective field (121) reduces to 


$$
\mathbf{H}_{i}^{(1)}(t)=-\frac{1}{M_{s i} V_{i}} \sum_{\kappa} \varepsilon_{\kappa} \partial_{\mathbf{m}_{i}} n_{\kappa}\left(\mathbf{m}_{i}, t\right) .
$$

The bulk damping coefficient (116) determined by the transverse spin-relaxation time $T_{2}$ in a model discussed in Sec. VI.B, where the average electron spin density lagged behind that corresponding to the instantaneous equilibrium, can be understood to arise from such an effective field. Spin-orbit interaction in the presence of a crystal field in bulk ferromagnets and/or exchange coupling through the normal spacer modulate $\varepsilon_{\kappa}\left(\mathbf{m}_{i}\right)$ via the time-dependent $\mathbf{m}_{i}$, leading to an additional dynamic contribution to the effective field, as follows from Eq. (121). This contribution has a particularly simple form in the limit of short relaxation times $\tau$ (Kunes and Kamberský, 2002; Heinrich, Urban, et al., 2003):

$$
\mathbf{H}_{i}^{(2)}(t)=-\frac{\tau}{M_{s i} V_{i}} \sum_{\kappa} \delta\left(\varepsilon_{\kappa}-\varepsilon_{F}\right) \frac{\partial \varepsilon_{\kappa}}{\partial \mathbf{m}_{i}}\left(\frac{\partial \varepsilon_{\kappa}}{\partial \mathbf{m}_{i}} \cdot \frac{d \mathbf{m}_{i}}{d t}\right),
$$

where $\varepsilon_{F}$ is the Fermi energy and assuming low temperature. It is clear that this effective field results in a (tensor) Gilbert damping.

In disordered structures and/or thick spacer layers with vanishing static exchange coupling between magnetic films, Eq. (121) should in principle reproduce the dynamic exchange coupling discussed in Sec. V. In other words, the spin pumping captures the semiclassical component of time-dependent exchange coupling between ferromagnetic films when the static contribution vanishes.

\section{B. Spin-orbit coupling}

The derivation of the spin-pumping current in Sec. III relies on Eqs. (21) and (22), which relate the scattering matrix in spin space, $\hat{s}_{n n^{\prime}, l l^{\prime}}$, for given channel and lead indices, $n, n^{\prime}$, and $l, l^{\prime}$, to the magnetization direction $\mathbf{m}$. For systems isotropic in spin space, the scattering matrix depends on $\mathbf{m}$ only through the simple projection (22). In transition-metal ferromagnets, this is a good approximation since their exchange splitting is by far the largest relevant energy scale. A large spin-orbit coupling in the electronic structure, such as in $p$-doped magnetic semiconductors like (Ga,Mn)As, on the other hand, can considerably modify the spin-pumping currents.

In ferromagnets with spin-orbit interaction, the rotating-frame analysis of Sec. III.B becomes tedious because of the need to apply the transformation to the orbital as well as spin degrees of freedom. Crystal anisotropies or even the presence of planar interfaces would make such an approach impractical. The adiabatic-pumping formalism of Sec. III.A, however, still applies. One may in general calculate the tensor current (45) in terms of the emissivity (46) when the dependence of the full scattering matrix on the magnetization direction is known. Of course, the $2 \times 2$ matrices have to be generalized to $(2 S+1) \times(2 S+1)$ for spin- $S$ carriers. In the case of spin-orbit coupling in nonmagnetic leads, however, such tensor currents are in general different from spin currents, since spin is not a good quantum number for transverse quantum channels. This complicates the matter beyond the scope of this article. In the simplest case of weak spin-orbit coupling within the ferromagnets, Eq. (21) holds approximately near a given m with weakly m-dependent scattering coefficients $s^{\uparrow(\downarrow)}$ for spin-1/2 carriers. The adiabatic spin-pumping current is then given by Eq. (38) in terms of a possibly m-dependent $\mathcal{A}^{\uparrow \downarrow}$.

For ferromagnets with a strong spin-orbit coupling, the most general form for the adiabatic spin-pumping current is

$$
I_{s, a}=\sum_{a a^{\prime}} \mathcal{A}_{a a^{\prime}}(\mathbf{M}) \frac{d M_{a^{\prime}}}{d t},
$$

with constraints on the form of the $3 \times 3$ tensor $\mathcal{A}_{a a^{\prime}}(\mathbf{M})$, $a, a^{\prime}=1,2,3$, for a given crystal symmetry. In particular, $I_{s, a}$ may now have a component along the magnetization direction. It is always the case, for example, when the ferromagnetic exchange field varies in magnitude as well as in direction. By conservation of the total angular momentum, the spin-pumping current (124) is accompanied by a torque on magnetization and also transferred into the orbital angular momentum as well as lattice torque. Note that in the presence of magnetic anisotropies, the Landau-Lifshitz-Gilbert equation of motion is a tensor equation in which the scalar Gilbert parameter $\alpha$ is replaced by a $3 \times 3$ tensor (Mills and Rezende, 2003).

\section{Inhomogeneous magnetization dynamics}

We have so far restricted our attention to spatially uniform magnetization dynamics. Recall, for example, Eq. (75) for spin-pumping-enhanced Gilbert damping in $F \mid N$ heterostructures. Several authors recently discussed possibilities for also accessing nonuniform spin-wave modes. Polianski and Brouwer (2004) have showed that for sufficiently large perpendicular-current densities, a single thin-film ferromagnet sandwiched between diffuse normal-metal contacts becomes unstable with respect to transverse (to the layering direction) excitations of finite-wavelength spin waves when the source and drain contacts are asymmetric and only for one direction (determined by the asymmetry) of the current bias. Their calculation is based on magnetoelectronic circuit theory and adiabatic spin pumping. Stiles et al. (2004) have considered the magnetic instability in diffuse $N|F| N$ structures in the limit of thicker $F$ layers that can undergo longitudinal (along the layering direction) as well as transverse magnetization dynamics, allowing the relaxation of the contact-asymmetry condition. Özyilmaz et al. (2004) have reported an experimental study of the current-induced excitations in $\mathrm{Cu}|\mathrm{Co}| \mathrm{Cu}$ nanopillars, qualitatively confirming the theoretical ideas. Brataas, Tserkovnyak, and Bauer (2005) and Özyilmaz et al. (2005) have investigated current-driven spin-wave instabilities in spin valves, in which they compete with the coherent macrospin motion. A detailed discussion of the current-induced instabilities is beyond the scope of the 
present review. We would nonetheless like to outline how Gilbert damping (75) is affected by a weak transverse spin-wave excitation in a single magnetic film in contact with a diffuse metal.

Consider a nonuniform transverse excitation of thinfilm magnetization in the limit of small amplitudes so that spin waves at different wavelengths do not couple. It is then sufficient to study excitations at a single wavelength with the magnetization direction deviating from its equilibrium value $\mathbf{m}_{0}$ as

$$
\mathbf{m}(\mathbf{r})-\mathbf{m}_{0}=\delta \mathbf{m} \cos \left(\mathbf{q}_{\perp} \cdot \boldsymbol{\rho}+\omega t+\varphi\right),
$$

where $\varphi$ is an arbitrary phase. The spatial position vector $\mathbf{r}$ is decomposed here into a coordinate along the layering direction $x$ and a transverse vector in the interface plane $\boldsymbol{\rho}$. The wave vector $\mathbf{q}_{\perp}$ of transverse spin waves is parallel to the $F \mid N$ interface and the amplitude $\delta \mathbf{m}$ does not depend on $x$. The derivation of the effective Gilbert damping for transverse spin waves (Polianski and Brouwer, 2004) closely resembles that in Sec. IV.B for the macrospin dynamics. Let us consider a normal layer capping one side of the ferromagnet. The presence of two normal layers sandwiching a magnetic film thicker than its coherence length $\lambda_{\mathrm{sc}}$ simply doubles the effect. For an unbiased structure, there is no charge current or voltage imbalance as long as the dynamics are slow on the characteristic spin-relaxation time scales, $\omega$ $\ll \tau_{\text {sf }}^{-1}$, which is assumed in the following. In order to find the enhanced Gilbert damping, the diffusion equation for spins in the normal metal (65) must be solved with the boundary condition (67). In contrast to Sec. IV.B, the spin-pumping current now depends on the transverse coordinate $\boldsymbol{\rho}$. The solution of the diffusion equation for the spin accumulation in the normal-metal film with thickness $L$ as a function of distance $x$ from the $F \mid N$ interface is [cf. Eq. (68)]

$$
\begin{aligned}
\boldsymbol{\mu}_{s}(x, \boldsymbol{\rho})= & \cosh \left[(x-L) / \lambda_{\mathrm{sd}}^{(\mathrm{eff})}\right] / \sinh \left[L / \lambda_{\mathrm{sd}}^{(\mathrm{eff})}\right] \\
& \times \frac{2 \lambda_{\mathrm{sd}}^{(\mathrm{eff})}}{\hbar \mathcal{N S} D} \cos \left(\mathbf{q}_{\perp} \cdot \boldsymbol{\rho}+\omega t+\varphi\right) \mathbf{I}_{s},
\end{aligned}
$$

where the effective spin-diffusion length for the transverse mode is defined by

$$
\lambda_{\mathrm{sd}}^{(\mathrm{eff})}=\frac{\lambda_{\mathrm{sd}}}{\sqrt{1+\left(\lambda_{\mathrm{sd}} \mathbf{q}_{\perp}\right)^{2}}},
$$

which reduces to the usual $\lambda_{\text {sd }}$, Eq. (70), for uniform dynamics. Using a calculation similar to that of Sec. IV.B, the wave-vector dependence of the enhanced Gilbert damping that generalizes Eq. (75) reads (Polianski and Brouwer, 2004)

$G_{\mathrm{eff}}\left(\mathbf{q}_{\perp}\right)-G=\left[1+\tilde{g}_{r}^{\uparrow} \frac{R_{\mathrm{sd}} \lambda_{\mathrm{sd}}^{(\text {eff })} / \lambda_{\mathrm{sd}}}{\tanh \left(L / \lambda_{\mathrm{sd}}^{(\text {eff })}\right)}\right]^{-1} \frac{\hbar \gamma^{2} \tilde{g}_{r}^{\uparrow \downarrow}}{4 \pi V}$.

Thick layers with $L \gg \lambda_{\text {sd }}^{(\text {eff })}$ are thus the best spin sinks for a given material composition and spin-wave wave vector.
There is a crossover in the behavior of the enhanced Gilbert damping when the wavelength is comparable to the spin-diffusion length. In the long-wavelength limit, $\lambda_{\mathrm{sw}} \gg 2 \pi \lambda_{\mathrm{sd}}\left(\lambda_{\mathrm{sw}}=2 \pi / q_{\perp}\right)$, the result reduces to that of a uniformly precessing ferromagnet; for short-wavelength excitations, $\lambda_{\text {sw }} \ll 2 \pi \lambda_{\text {sd }}$, the damping depends on the wavelength corresponding to the reduced effective spindiffusion length $\lambda_{\mathrm{sd}}^{\text {(eff) }} \approx \lambda_{\mathrm{sw}} / 2 \pi$. In the latter regime, numerical estimates for transition-metal ferromagnets in contact with simple normal metals, in the spirit of Sec. IV.B, give

$$
G_{\text {eff }}\left(\lambda_{\mathrm{sw}}\right)-G \sim \frac{\hbar \gamma^{2} \tilde{g}_{r}^{\uparrow} \downarrow / 4 \pi V}{1+\left[4 \lambda / \lambda_{\mathrm{sw}} \tanh \left(2 \pi L / \lambda_{\mathrm{sw}}\right)\right]^{-1}},
$$

where $\lambda$ is the transport mean free path. We thus find that a normal metal is always a good spin sink in the limit $\lambda_{\text {sw }} \ll \lambda<L$, independent of spin-relaxation rates, in stark contrast to the long-wavelength result (76). This can be understood by referring to the discussion of dynamic exchange coupling in Sec. V.A and noticing that the pumping and backflow reabsorption of spins are separated in space by distances of the order or larger than $\lambda$, corresponding to regions of the magnetic layer with dynamics that are incoherent upon averaging over various diffuse paths when $\lambda_{\text {sw }} \ll \lambda$. The damping of each magnetic region is therefore affected by the spin pumping with vanishing spin backflow, rendering the normal metal a good spin sink. Consequently, the efficiency of the normal metal as a spin sink increases for shorterwavelength spin-wave excitations and longer mean free paths. In particular, the Gilbert damping can be enhanced even when there is no enhancement of the Gilbert damping for long-wavelength excitations. This general conclusion can also be extended to magnetic films that are inhomogeneous or disordered on length scales shorter than the transport mean free path in nonmagnetic buffers. In addition, the normal metal may become an efficient spin sink for both short- and longwavelength spin waves when the frequency of these excitations becomes larger than the normal-metal scattering rate, a regime not explicitly treated in this review. (The interfacial spin-pumping current can still be evaluated by the adiabatic formalism as long as the frequency remains much smaller than the ferromagnetic exchange energy.) Finally, we remark that quite generally, in the limit when the normal metal effectively becomes an ideal spin sink, the mixing conductance that determines the strength of the spin-pumping current should not be renormalized (following the Sec. II.B discussion). In particular, it is $g^{\uparrow \downarrow}$ and not $\tilde{g}^{\uparrow \downarrow}$ that enters Eq. (129) when the denominator on the right-hand side is close to unity. In the intermediate spin-sink regime, one has to extend the discussion of Sec. II.B to laterally inhomogeneous systems.

\section{Electron-electron interactions}

The appropriate framework for describing metallic magnetism, including the $3 d$ transition metals, is band 
theory (Kübler, 2000), treating the electron-electron interaction in a mean-field approximation. For qualitative purposes, this comes down to a simple Stoner or $s-d$ model Hamiltonian having parabolic free-electron dispersion for conduction electrons with parametrized masses and exchange splittings. The local-spin-density approximation of density-functional theory is a very successful framework for treating itinerant electron systems from first principles. Electron-correlation effects are taken into account by the exchange-correlation potentials, be it in the local approximation. This approach has been taken by, e.g., Zwierzycki et al. (2005) in calculating the scattering matrix and the conductance parameters.

Šimánek (2003) and Šimánek and Heinrich (2003) have raised the question of possible enhancement of spin pumping in metallic $F \mid N$ heterostructures by electron-electron interactions in the normal metals. A potential candidate would be $\mathrm{Pd}$ as a normal metal with an interaction-enhanced magnetic susceptibility. $\mathrm{Pd}$ is "nearly" ferromagnetic causing, e.g., giant moments around magnetic impurities. A ferromagnetic film in contact with such a material can thus induce magnetic moments renormalizing the exchange potential felt by electrons at the $F \mid N$ contact. Simánek (2003) and Simánek and Heinrich (2003) have considered the problem of an ultrathin " $\delta$-function" magnetic layer embedded in a nonmagnetic material with a large Stoner enhancement of the spin susceptibility. Treating the ferromagnetic exchange field as a perturbation felt by normal-metal electrons, one can obtain a significant enhancement of its effective mean-field profile by electronelectron interactions. This in turn can considerably increase the spin-mixing conductances that govern the spin pumping (Šimánek, 2003). Such perturbative analysis, however, overestimates the effect of electron correlations on spin-mixing conductances and thereby spin pumping in transition-metal heterostructures. It has been explained in Sec. VII.A.1 that the mixing conductance $g^{\uparrow \downarrow}$ (computed nonperturbatively by densityfunctional theory) of even the thinnest (and more so the thicker) magnetic films is close to the normal-metal Sharvin conductance, regardless of the possible Stoner enhancement (Zwierzycki et al., 2005). The mixing conductance that determines the strength of spin pumping is thus not expected to be correlated with the normalmetal spin susceptibility, as already emphasized in Sec. IV.A.

In order to understand that a Stoner enhancement does not directly affect the spin-pumping enhancement, it is convenient to perform the rotating-frame analysis of Sec. III.B, which is also valid in the presence of electronelectron interactions and invariant to the rotating-frame transformation. The precessing ferromagnet changes the polarization in the normal metal along the axis of rotation by an amount which corresponds to the spinimbalance potential $\mu_{s}=\hbar \omega$ that is determined by the precession frequency $\omega$, irrespective of the materials under consideration. In particular, the steady-state spin accumulation $\mu_{s}$ due to spin pumping does not depend on the magnetic susceptibility. Since it is $\mu_{s}$ that drives the nonequilibrium spin flow and not the spin density (that is affected by the Stoner enhancement), the arguments given before in this article remain valid for interacting systems treated within the mean-field picture.

The electron-electron effects such as an enhanced normal-metal susceptibility are implicitly and nonperturbatively taken into account in self-consistent $a b$ initio calculations of heterostructures. First-principles calculations of the scattering matrix (Zwierzycki et al., 2005) fully include the electron-correlation effects which are difficult to capture with a perturbative formalism. Although many researchers in the field of solid-state magnetism are not familiar with scattering theory, it appears to be the most natural language for pursuing the study of coupled transport and magnetization dynamics.

Whereas electron-electron effects beyond the meanfield local-spin-density approximation are probably small for transition metals, this does not mean that they can be neglected in other systems. For example, a suppression of spin pumping by correlation effects in onedimensional metals ("Luttinger liquids") has been predicted by Bena and Balents (2004). These authors found that the spin pumping by a moving ferromagnet into a Luttinger liquid through a tunnel barrier is given by the same expression as for noninteracting electrons, viz., Eq. (38), but with parameters $\mathcal{A}_{r}^{\uparrow \downarrow}$ and $\mathcal{A}_{i}^{\uparrow \downarrow}$ vanishing as a power of temperature at low temperatures with an exponent characteristic for the Luttinger-liquid zero-bias anomaly in tunneling density of states. Correlation effects might also become important if one wishes to describe spin pumping close to the ferromagnetic critical temperature, to understand significant deviations from the macrospin model for the magnetization, and to quantitatively model the spin-pumping parameter for interfaces with strongly correlated materials.

\section{SUMMARY AND OUTLOOK}

In this review we have presented a coherent picture of nonlocal magnetization dynamics in heterostructures of ferromagnets and nonmagnetic conductors. It is based on the assumption of semiclassical transport in bulk materials that is valid for diffuse and chaotic systems, as well as a separation of time scales of the electronic and magnetic degrees of freedom. Interfaces are treated as sharp quantum-mechanical boundary conditions for electron distribution functions and nonequilibrium transport. Except for the phenomenological treatment of spin-flip scattering processes, the theory is derived from first principles. The main subject in this context is the concept of spin pumping due to moving magnetization vectors. The magnetization dynamics affected by the spin-transfer torque in the presence of an electrical bias should be treated on an equal footing with spin pumping. The crucial material parameter is the spinmixing conductance that can be computed from ab initio electronic band structures.

Several phenomena can be predicted or explained based on the basic formalism. One of them is the increased Gilbert damping of thin magnetic films in good 
electrical contact with normal metals that efficiently dissipate spin angular momentum. In more complex magnetic structures, we predict an interplay between spin pumping and magnetization torques that is truly nonlocal, i.e., depends on the entire spin-coherent volume of the sample (determined by the spin-flip diffusion length). Novel collective effects appear when different magnetic elements in a spin-coherent circuit or device resonate at nearby frequencies. A moving ferromagnet can be used as a source that pumps spin currents into normal metals or semiconductors, which leads to a spin accumulation determined by the ferromagneticresonance frequency.

Although some basic principles are rather subtle, the final formalism is easy to use. It can often be mapped on an equivalent circuit model that is governed by a few parameters that can be either fitted to experiments or computed from first principles. Such calculations can be used, e.g., to estimate and optimize critical magnetization-switching currents (Manschot et al., 2004). We have focused attention on quasi-one-dimensional configurations and the macrospin model for the magnetization. Generalization of the formalism to include spin-wave excitations in the ferromagnet or inhomogeneous spin currents have also been illustrated (Sec. VII.C). Integration of micromagnetic simulations with the transport equations based on boundary conditions at the interfaces as described here might be necessary to improve the accuracy of first-principles modeling.

In this review we had mainly metallic heterostructures with transition-metal ferromagnets in mind. But since the approach is quite general, we should by no means exclude other materials. We have already speculated in Sec. VI.B that the formalism can be used to understand Gilbert damping in magnetic semiconductors. There is little doubt that a modeling of the current-induced switching of (Ga,Mn)As observed by Chiba et al. (2004) requires the concept of magnetization torque. Spin pumping into carbon nanotubes as investigated by Bena and Balents (2004) is partly suppressed by correlation effects. Spin pumping by ferromagnetic superconductors (Brataas and Tserkovnyak, 2004) is entangled with Cooper-pair pumping and depends on the spin-pairing symmetry of the superconducting state. Spin dynamics in heterostructures of high-density magnets in contact with doped semiconductors (Bauer et al., 2004, 2005) are another promising playground for the formalism described here. Besides, it is possible to generalize the spin-pumping picture to other symmetry-broken heterostructures with adiabatically varying order parameters (Tserkovnyak and Brataas, 2005).

A critical parameter in nonlocal magnetization dynamics is the spin-flip diffusion length in normal-metal components, which can be of the order of microns even at room temperature; see, e.g., Jedema et al. (2002). Nonlocal effects in structures based on transition metals could therefore be robust and observable at ambient temperatures. We therefore believe that the formalism discussed here should be useful for understanding, computing, and designing magnetic-device operations. An example is the possibility of " $\alpha$ engineering" based on the increase of the Gilbert damping in ultrathin magnetic films, e.g., by an order of magnitude, by just evaporating a few monolayers of $\mathrm{Pt}$ on top of it. We also expect that with the decreasing size of magnetic circuits and devices, the dynamic coupling discussed in this review will become even more relevant. It might lead to effects like cross talk between magnetic elements and excess noise. On the other hand, proper engineering of phenomena like the nonlocal dynamic locking might lead to an increased stability against external perturbations as well.

Theoretical challenges for the future include a proper treatment of spin-orbit interactions, the coupling of magnetic degrees of freedom to the lattice, and effects beyond the semiclassical regime.

\section{ACKNOWLEDGMENTS}

We are grateful to G. A. Fiete, B. Heinrich, P. J. Kelly, and M. Zwierzycki for contributing to this review and to J. Foros and M. D. Stiles for valuable discussions. This work was supported in part by the Harvard Society of Fellows, the Norwegian Research Council Grant No. 162742/V00, Nanomat Grant Nos. 158518/143 and 158547/431, Dutch FOM Foundation, DARPA Award No. MDA972-01-1-0024, and NSF Grant Nos. DMR 0233773 and PHY 01-17795.

\section{REFERENCES}

Abrikosov, A. A., and L. P. Gor'kov, 1962, Zh. Eksp. Teor. Fiz. 42, 1088 [Sov. Phys. JETP 15, 752 (1962)].

Akkermans, E., A. Auerbach, J. E. Avron, and B. Shapiro, 1991, Phys. Rev. Lett. 66, 76.

Apalkov, D. M., and P. B. Visscher, 2004, e-print cond-mat/ 0405305.

Baibich, M. N., J. M. Broto, A. Fert, F. N. V. Dau, F. Petroff, P. Etienne, G. Creuzet, A. Friedrich, and J. Chazelas, 1988, Phys. Rev. Lett. 61, 2472.

Barnes, S. E., 1974, J. Phys. F: Met. Phys. 4, 1535.

Barnes, S. E., and S. Maekawa, 2005, Phys. Rev. Lett. 95, 107204.

Bass, J., and W. P. Pratt, Jr., 1999, J. Magn. Magn. Mater. 200, 274.

Bastian, D., and E. Biller, 1976, Phys. Status Solidi A 35, 113. Bauer, G. E. W., A. Brataas, Y. Tserkovnyak, B. I. Halperin, M. Zwierzycki, and P. J. Kelly, 2004, Phys. Rev. Lett. 92, 126601.

Bauer, G. E. W., Y. Tserkovnyak, A. Brataas, J. Ren, K. Xia, M. Zwierzycki, and P. J. Kelly, 2005, e-print cond-mat/ 0501726.

Bauer, G. E. W., Y. Tserkovnyak, D. Huertas-Hernando, and A. Brataas, 2003a, in Advances in Solid State Physics, edited by B. Kramer (Springer-Verlag, Heidelberg), Vol. 43, p. 383. Bauer, G. E. W., Y. Tserkovnyak, D. Huertas-Hernando, and A. Brataas, 2003b, Phys. Rev. B 67, 094421.

Beenakker, C. W. J., 1997, Rev. Mod. Phys. 69, 731.

Bena, C., and L. Balents, 2004, Phys. Rev. B 70, 245318.

Berger, L., 1996, Phys. Rev. B 54, 9353.

Berger, L., 2001, J. Appl. Phys. 90, 4632. 
Bergmann, G., 1982, Z. Phys. B: Condens. Matter 48, 5.

Bhagat, S. M., and P. Lubitz, 1974, Phys. Rev. B 10, 179.

Binasch, G., P. Grünberg, F. Saurenbach, and W. Zinn, 1989, Phys. Rev. B 39, 4828.

Brataas, A., G. E. W. Bauer, and P. J. Kelly, 2005, unpublished. Brataas, A., Y. V. Nazarov, and G. E. W. Bauer, 2000, Phys. Rev. Lett. 84, 2481.

Brataas, A., Y. V. Nazarov, and G. E. W. Bauer, 2001, Eur. Phys. J. B 22, 99.

Brataas, A., and Y. Tserkovnyak, 2004, Phys. Rev. Lett. 93, 087201.

Brataas, A., Y. Tserkovnyak, and G. E. W. Bauer, 2005, e-print cond-mat/0501672.

Brataas, A., Y. Tserkovnyak, G. E. W. Bauer, and B. I. Halperin, 2002, Phys. Rev. B 66, 060404(R).

Brataas, A., G. Zaránd, Y. Tserkvovnyak, and G. E. W. Bauer, 2003, Phys. Rev. Lett. 91, 166601.

Brouwer, P. W., 1998, Phys. Rev. B 58, R10135.

Brown, W. F., Jr., 1963, Micromagnetics (Wiley, New York). Bruno, P., 1995, Phys. Rev. B 52, 411.

Büttiker, M., H. Thomas, and A. Prêtre, 1994, Z. Phys. B: Condens. Matter 94, 133.

Camley, R. E., and J. Barnas, 1989, Phys. Rev. Lett. 63, 664.

Camley, R. E., and R. L. Stamps, 1993, J. Phys.: Condens. Matter 5, 3727.

Capelle, K., G. Vignale, and B. L. Györffy, 2001, Phys. Rev. Lett. 87, 206403.

Chiang, W.-C., C. Ritz, K. Eid, R. Loloee, W. P. Pratt, Jr., and J. Bass, 2004, Phys. Rev. B 69, 184405.

Chiba, D., Y. Sato, T. Kita, F. Matsukura, and H. Ohno, 2004, Phys. Rev. Lett. 93, 216602.

Covington, M., M. AlHajDarwish, Y. Ding, N. J. Gokemeijer, and M. A. Seigler, 2004, Phys. Rev. B 69, 184406.

Demokritov, S., and E. Tsymbal, 1994, J. Phys.: Condens. Matter 6, 7145.

Dietl, T., H. Ohno, and F. Matsukura, 2001, Phys. Rev. B 63, 195205.

Dobin, A. Y., and R. H. Victora, 2003, Phys. Rev. Lett. 90, 167203.

D'yakonov, M. I., and V. I. Perel, 1971, Zh. Eksp. Teor. Fiz. 60, 1954 [Sov. Phys. JETP 33, 1053 (1971)].

Engels, G., J. Lange, T. Schäpers, and H. Lüth, 1997, Phys. Rev. B 55, R1958.

Erickson, R. P., K. B. Hathaway, and J. R. Cullen, 1993, Phys. Rev. B 47, 2626.

Fert, A., and L. Piraux, 1999, J. Magn. Magn. Mater. 200, 338. Fiederling, R., M. Keim, G. Reuscher, W. Ossau, G. Schmidt, A. Waag, and L. W. Molenkamp, 1999, Nature (London) 402, 787.

Fitzsimmons, M. R., et al., 2004, J. Magn. Magn. Mater. 271, 103.

Foros, J., A. Brataas, Y. Tserkovnyak, and G. E. W. Bauer, 2005, Phys. Rev. Lett. 95, 016601.

Foros, J., G. Woltersdorf, B. Heinrich, and A. Brataas, 2005, J. Appl. Phys. 97, 10A714.

Gijs, M. A. M., and G. E. W. Bauer, 1997, Adv. Phys. 46, 285.

Gijs, M. A. M., S. K. J. Lenczowski, and J. B. Giesbers, 1993, Phys. Rev. Lett. 70, 3343.

Gilbert, T. L., 1955, Phys. Rev. 100, 1243.

Gilbert, T. L., 2004, IEEE Trans. Magn. 40, 3443.

Goennenwein, S. T. B., et al., 2003, Appl. Phys. Lett. 82, 730.

Grünberg, P., R. Schreiber, Y. Pang, M. B. Brodsky, and H.

Sowers, 1986, Phys. Rev. Lett. 57, 2442.
Gunnarsson, O., 1976, J. Phys. F: Met. Phys. 6, 587.

Heinrich, B., and J. F. Cochran, 1993, Adv. Phys. 42, 523.

Heinrich, B., D. Fraitová, and V. Kamberský, 1967, Phys. Status Solidi 23, 501.

Heinrich, B., Y. Tserkovnyak, G. Woltersdorf, A. Brataas, R. Urban, and G. E. W. Bauer, 2003, Phys. Rev. Lett. 90, 187601. Heinrich, B., R. Urban, and G. Woltersdorf, 2002, J. Appl. Phys. 91, 7523.

Heinrich, B., R. Urban, G. Woltersdorf, and E. Šimánek, 2003, J. Appl. Phys. 93, 7545.

Heinrich, B., K. B. Urquhart, A. S. Arrott, J. F. Cochran, K. Myrtle, and S. T. Purcell, 1987, Phys. Rev. Lett. 59, 1756.

Hilton, D. J., and C. L. Tang, 2003, Phys. Rev. Lett. 89, 146601.

Huertas-Hernando, D., Y. V. Nazarov, and W. Belzig, 2002, Phys. Rev. Lett. 88, 047003.

Hurdequint, H., 1991, J. Magn. Magn. Mater. 93, 336.

Hurdequint, H., and G. Dunifer, 1988, J. Phys. (Paris), Colloq. 49, C8-1717.

Hurdequint, H., and M. Malouche, 1991, J. Magn. Magn. Mater. 93, 276.

Imry, Y., 1997, Introduction to Mesoscopic Physics (Oxford University Press, Oxford).

Ingvarsson, S., L. Ritchie, X. Y. Liu, G. Xiao, J. C. Slonczewski, P. L. Trouilloud, and R. H. Koch, 2002, Phys. Rev. B 66, 214416.

Janak, J. F., 1977, Phys. Rev. B 16, 255.

Janossy, A., 1980, Phys. Rev. B 21, 3793.

Janossy, A., and P. Monod, 1976, Phys. Rev. Lett. 37, 612.

Jedema, F. J., A. T. Filip, and B. J. van Wees, 2001, Nature (London) 410, 345.

Jedema, F. J., H. B. Heersche, A. T. Filip, J. J. A. Baselmans, and B. J. van Wees, 2002, Nature (London) 416, 713.

Ji, Y., C. L. Chien, and M. D. Stiles, 2003, Phys. Rev. Lett. 90, 106601.

Johnson, M., and R. H. Silsbee, 1988, Phys. Rev. B 37, 5312.

Kasuya, T., 1956, Prog. Theor. Phys. 16, 4558.

Katine, J. A., F. J. Albert, R. A. Buhrman, E. B. Myers, and D. C. Ralph, 2000, Phys. Rev. Lett. 84, 3149.

Kawakami, R. K., Y. Kato, M. Hanson, I. Malajovich, J. M. Stephens, E. Johnston-Halperin, G. Salis, A. C. Gossard, and D. D. Awschalom, 2001, Science 294, 131.

Kikkawa, J. M., and D. D. Awschalom, 1998, Phys. Rev. Lett. 80, 4313.

Kikkawa, J. M., and D. D. Awschalom, 1999, Nature (London) 397, 139.

Kim, J.-V., and C. Chappert, 2005, J. Magn. Magn. Mater. 286, 56.

Kiselev, S. I., J. C. Sankey, I. N. Krivorotov, N. C. Emley, R. J. Schoelkopf, R. A. Buhrman, and D. C. Ralph, 2003, Nature (London) 425, 380.

Korenman, V., and R. E. Prange, 1972, Phys. Rev. B 6, 2769.

Kovalev, A. A., A. Brataas, and G. E. W. Bauer, 2002, Phys. Rev. B 66, 224424.

Krivorotov, I. N., N. C. Emley, J. C. Sankey, S. I. Kiselev, D. C. Ralph, and R. A. Buhrman, 2005, Science 307, 228.

Kübler, J., 2000, Theory of Itinerant Electron Magnetism (Oxford University Press, Oxford).

Kunes, J., and V. Kamberský, 2002, Phys. Rev. B 65, 212411.

Lagae, L., R. Wirix-Speetjens, W. Eyckmans, S. Borghs, and J. De Boeck, 2005, J. Magn. Magn. Mater. 286, 291.

Landau, L. D., E. M. Lifshitz, and L. P. Pitaevski, 1980, Statistical Physics, Part 2, 3rd ed. (Pergamon, Oxford).

Landauer, R., 1970, Philos. Mag. 21, 863. 
Lee, K. J., A. Deac, O. Redon, J. P. Nozieres, and B. Dieny, 2004, Nat. Mater. 3, 877.

Lenz, K., T. Toliński, J. Lindner, E. Kosubek, and K. Baberschke, 2004, Phys. Rev. B 69, 144422.

Li, Z., and S. Zhang, 2004a, Phys. Rev. Lett. 92, 207203.

Li, Z., and S. Zhang, 2004b, Phys. Rev. B 69, 134416.

Lubitz, P., S. F. Cheng, and F. J. Rachford, 2003, J. Appl. Phys. 93, 8283 .

Lutovinov, V. S., and M. Y. Reizer, 1979, Zh. Eksp. Teor. Fiz. 77, 707 [Sov. Phys. JETP 50, 355 (1979)].

Luttinger, J. M., 1956, Phys. Rev. 102, 1030.

Mal'shukov, A. G., and K. A. Chao, 2000, Phys. Rev. B 61, R2413.

Manschot, J., A. Brataas, and G. E. W. Bauer, 2004, Appl. Phys. Lett. 85, 3250.

Meservey, R., and P. M. Tedrow, 1978, Phys. Rev. Lett. 41, 805. Mills, D. L., 2003, Phys. Rev. B 68, 014419.

Mills, D. L., and S. M. Rezende, 2003, in Spin Dynamics in Confined Magnetic Structures II, edited by B. Hillebrands and K. Ounadjela (Springer-Verlag, New York).

Miltat, J., G. Albuquerque, and A. Thiaville, 2002, in Spin Dynamics in Confined Magnetic Structures I, edited by B. Hillebrands and K. Ounadjela (Springer-Verlag, New York).

Mitchell, A. H., 1957, Phys. Rev. 105, 1439.

Miyazaki, T., and N. Tezuka, 1995, J. Magn. Magn. Mater. 139, L231.

Mizukami, S., Y. Ando, and T. Miyazaki, 2001a, J. Magn. Magn. Mater. 226, 1640.

Mizukami, S., Y. Ando, and T. Miyazaki, 2001b, Jpn. J. Appl. Phys., Part 1 40, 580.

Mizukami, S., Y. Ando, and T. Miyazaki, 2002a, Phys. Rev. B 66, 104413.

Mizukami, S., Y. Ando, and T. Miyazaki, 2002b, J. Magn. Magn. Mater. 239, 42.

Monod, P., H. Hurdequint, A. Janossy, J. Obert, and J. Chaumont, 1972, Phys. Rev. Lett. 29, 1327.

Monsma, D., R. Vlutters, and J. Lodder, 1998, Science 281, 407.

Moodera, J. S., L. R. Kinder, T. Wong, and R. Meservey, 1995, Phys. Rev. Lett. 74, 3273.

Myers, E. B., F. J. Albert, J. C. Saneky, E. Bonet, R. A. Buhrman, and D. C. Ralph, 2002, Phys. Rev. Lett. 89, 196801.

Myers, E. B., D. C. Ralph, J. A. Katine, R. N. Louie, and R. A. Buhrman, 1999, Science 285, 867.

Nitta, J., T. Akazaki, H. Takayanagi, and T. Enoki, 1997, Phys. Rev. Lett. 78, 1335.

Ohno, H., 1999, J. Magn. Magn. Mater. 200, 110.

Ohno, Y., D. K. Young, B. Beschoten, F. Matsukura, H. Ohno, and D. D. Awschalom, 1999, Nature (London) 402, 790.

Overhauser, A. W., 1953, Phys. Rev. 92, 411.

Özyilmaz, B., A. D. Kent, D. Monsma, J. Z. Sun, M. J. Rooks, and R. H. Koch, 2003, Phys. Rev. Lett. 91, 067203.

Özyilmaz, B., A. D. Kent, M. J. Rooks, and J. Z. Sun, 2005, Phys. Rev. B 71, 140403(R).

Özyilmaz, B., A. D. Kent, J. Z. Sun, M. J. Rooks, and R. H. Koch, 2004, Phys. Rev. Lett. 93, 176604.

Paget, D., G. Lampel, B. Sapoval, and V. I. Safarov, 1977, Phys. Rev. B 15, 5780.

Parkin, S. S. P., N. More, and K. P. Roche, 1990, Phys. Rev. Lett. 64, 2304.

Parks, P. D., and R. H. Silsbee, 1987, Phys. Rev. B 35, 5198.

Patton, C. E., Z. Frait, and C. H. Wilts, 1975, J. Appl. Phys. 46, 5002 .
Piraux, L., S. Dubois, and A. Fert, 1996, J. Magn. Magn. Mater. 159, L287.

Polianski, M. L., and P. W. Brouwer, 2004, Phys. Rev. Lett. 92, 026602.

Pratt, W. P., Jr., S.-F. Lee, J. M. Slaughter, R. Loloee, P. A. Schroeder, and J. Bass, 1991, Phys. Rev. Lett. 66, 3060.

Pufall, M. R., W. H. Rippard, S. Kaka, S. E. Russek, T. J. Silva, J. Katine, and M. Carey, 2004, Phys. Rev. B 69, 214409.

Qian, Z., and G. Vignale, 2002, Phys. Rev. Lett. 88, 056404.

Rappoport, T. G., P. Redliński, X. Liu, G. Zaránd, J. K. Furdyna, and B. Jankó, 2004, Phys. Rev. B 69, 125213.

Rippard, W. H., M. R. Pufall, S. Kaka, S. E. Russek, and T. J. Silva, 2004, Phys. Rev. Lett. 92, 027201.

Ruderman, M. A., and C. Kittel, 1954, Phys. Rev. 96, 99.

Safonov, V. L., and H. N. Bertram, 2000, Phys. Rev. B 61, R14893.

Schep, K. M., J. B. A. N. van Hoof, P. J. Kelly, G. E. W. Bauer, and J. E. Inglesfield, 1997, Phys. Rev. B 56, 10805.

Schmidt, G., D. Ferrand, L. W. Molenkamp, A. T. Filip, and B. J. van Wees, 2000, Phys. Rev. B 62, R4790.

Sharma, P., and P. W. Brouwer, 2003, Phys. Rev. Lett. 91, 166801.

Silsbee, R. H., A. Janossy, and P. Monod, 1979, Phys. Rev. B 19, 4382.

Šimánek, E., 2003, Phys. Rev. B 68, 224403.

Šimánek, E., 2004, e-print cond-mat/0405020.

Šimánek, E., and B. Heinrich, 2003, Phys. Rev. B 67, 144418.

Sinova, J., T. Jungwirth, X. Liu, Y. Sasaki, J. K. Furdyna, W. A. Atkinson, and A. H. MacDonald, 2004, Phys. Rev. B 69, 085209.

Slichter, C. P., 1990, Principles of Magnetic Resonance, 3rd ed. (Springer-Verlag, New York).

Slonczewski, J. C., 1989, Phys. Rev. B 39, 6995.

Slonczewski, J. C., 1993, J. Magn. Magn. Mater. 126, 374.

Slonczewski, J. C., 1996, J. Magn. Magn. Mater. 159, L1.

Slonczewski, J. C., 1999, J. Magn. Magn. Mater. 195, L261.

Stiles, M. D., 1999, J. Magn. Magn. Mater. 200, 322.

Stiles, M. D., J. Xiao, and A. Zangwill, 2004, Phys. Rev. B 69, 054408.

Stiles, M. D., and A. Zangwill, 2002, Phys. Rev. B 66, 014407. Sun, J. Z., B. Özyilmaz, W. Chen, M. Tsoi, and A. D. Kent, 2005, J. Appl. Phys. 97, 10C714.

Tatara, G., and H. Kohno, 2004, Phys. Rev. Lett. 92, 086601.

Tserkovnyak, Y., and A. Brataas, 2001, Phys. Rev. B 64, 214402.

Tserkovnyak, Y., and A. Brataas, 2005, Phys. Rev. B 71, 052406.

Tserkovnyak, Y., A. Brataas, and G. E. W. Bauer, 2002a, Phys. Rev. Lett. 88, 117601.

Tserkovnyak, Y., A. Brataas, and G. E. W. Bauer, 2002b, Phys. Rev. B 66, 224403.

Tserkovnyak, Y., A. Brataas, and G. E. W. Bauer, 2003a, J. Appl. Phys. 93, 7534.

Tserkovnyak, Y., A. Brataas, and G. E. W. Bauer, 2003b, Phys. Rev. B 67, 140404(R).

Tserkovnyak, Y., G. A. Fiete, and B. I. Halperin, 2004, Appl. Phys. Lett. 84, 5234.

Tsoi, M., A. G. M. Jansen, J. Bass, W.-C. Chiang, M. Seck, V. Tsoi, and P. Wyder, 1998, Phys. Rev. Lett. 80, 4281.

Tsoi, M., A. G. M. Jansen, J. Bass, W. C. Chiang, V. Tsoi, and P. Wyder, 2000, Nature (London) 406, 46.

Urazhdin, S., N. O. Birge, W. P. Pratt, Jr., and J. Bass, 2003, Phys. Rev. Lett. 91, 146803. 
Urban, R., G. Woltersdorf, and B. Heinrich, 2001, Phys. Rev. Lett. 87, 217204.

Valet, T., 2004, unpublished.

Valet, T., and A. Fert, 1993, Phys. Rev. B 48, 7099.

Waintal, X., and P. W. Brouwer, 2003, Phys. Rev. Lett. 91 247201.

Waintal, X., E. B. Myers, P. W. Brouwer, and D. C. Ralph, 2000, Phys. Rev. B 62, 12317.

Waintal, X., and O. Parcollet, 2005, Phys. Rev. Lett. 94, 247206.

Watson, S. K., R. M. Potok, C. M. Marcus, and V. Umansky, 2003, Phys. Rev. Lett. 91, 258301.

Wegrowe, J.-E., D. Kelly, T. Truong, P. Guittienne, and J.-P. Ansermet, 2001, Europhys. Lett. 56, 748.

Xia, K., P. J. Kelly, G. E. W. Bauer, A. Brataas, and I. Turek, 2002, Phys. Rev. B 65, 220401(R).
Xia, K., P. J. Kelly, G. E. W. Bauer, I. Turek, J. Kudrnovsky, and V. Drchal, 2001, Phys. Rev. B 63, 064407.

Yakata, S., Y. Ando, S. Mizukami, and T. Miyazaki, 2005, J. Magn. Soc. Jpn. (to be published).

Yang, Q., P. Holody, S.-F. Lee, L. L. Henry, R. Loloee, P. A. Schroeder, W. P. Pratt, Jr., and J. Bass, 1994, Phys. Rev. Lett. 72, 3274.

Yosida, K., 1957, Phys. Rev. 106, 893.

Yuasa, S., T. Nagahama, and Y. Suzuki, 2002, Science 297, 234.

Zhu, H. J., M. Ramsteiner, H. Kostial, M. Wassermeier, H.-P. Schonherr, and K. H. Ploog, 2001, Phys. Rev. Lett. 87, 016601.

Zwierzycki, M., Y. Tserkovnyak, P. J. Kelly, A. Brataas, and G. E. W. Bauer, 2005, Phys. Rev. B 71, 064420. 